\title{
ACOUSTIC EMISSION SPIKES IN GRINDING: ORIGIN AND APPLICATIONS
}




\title{
ACOUSTIC EMISSION SPIKES AT WORKPIECE \\ EDGES IN GRINDING: ORIGIN AND APPLICATIONS
}

By

RYAN J.P. BABEL, B.ENG.

\author{
A Thesis \\ Submitted to the School of Graduate Studies \\ In Partial Fulfillment of the Requirements \\ For the Degree \\ Master of Applied Science
}

McMaster University

(C) Copyright by Ryan J.P. Babel, September 2011 
MASTER OF APPLIED SCIENCE (2011)

(Department of Mechanical Engineering)
McMaster University

Hamilton, Ontario
TITLE:

AUTHOR:

SUPERVISOR:

NUMBER OF PAGES: Xiii, 122
Acoustic emission spikes at workpiece edges in grinding: Origin and applications Ryan J.P. Babel, B.Eng. (McMaster University) Dr. Philip Koshy 


\section{Abstract}

Unexplained acoustic emission spikes have been reported to occur at the workpiece entry and exit in interrupted machining operations, most notably during grinding. These AE spikes have yet to be fully explained in terms of their cause, or interpreted to yield useful information regarding the grinding process. This thesis research hence focuses on investigating this phenomenon. What started as a curiosity-driven project has resulted in an experimental technique to measure the actual contact length between the workpiece and the grinding wheel, as well as a technique for detecting grinding burn, which are of remarkable practical significance. 


\section{Acknowledgements}

The author would like to identify all individuals who have helped make this work possible and who have offered their kind support throughout this process.

First and foremost, I would like to recognize my supervisor, Dr. Philip Koshy. His innovative ideas and desire to pursue new and exciting research made working with him an unforgettable experience. I also wish to acknowledge his support and encouragement throughout this process and for treating each of his students as family. The level of independence and trust granted by Dr. Koshy not only impacted my project, but allowed me to mature as both an adult and future engineer. From guiding me through times of difficulty, to simply sharing a coffee, his influence on my time as a graduate has been second to none. Dr. Koshy exhibits a sense of support and community towards his students that directly translates into academic success. Thank you for everything!

I would also like to thank members of the shop; Terry, JP, Ron, Mark, Jim and Joe for their help with the everyday situations of performing experiments and working with new equipment.

Janet's kindness and assistance when it came to the dozens of purchase order applications is also something that must be recognized. I couldn’t imagine it going so smoothly without her!

Finally, but most importantly, I would like to thank my family. They have always supported me in everything that I do, and I am proud to share this accomplishment with them. 


\section{Table of Contents}

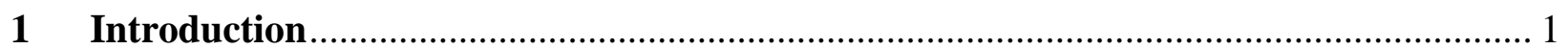

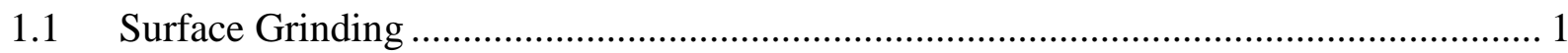

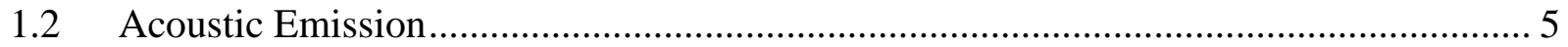

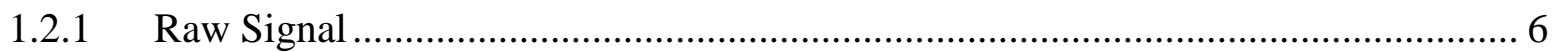

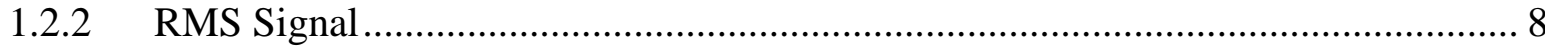

1.3 Scope and Organization of Present Work ............................................................... 8

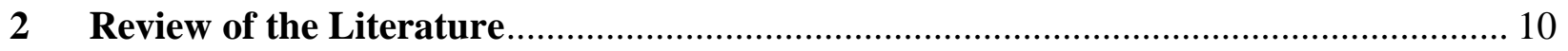

2.1 AE Entry/Exit Spike Phenomenon during Grinding ............................................... 10

2.1.1 AE Entry/Exit Spike Phenomenon during Cutting ............................................ 15

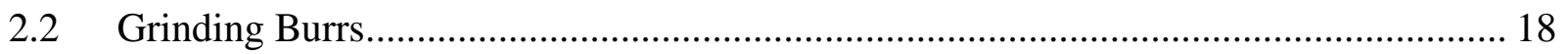

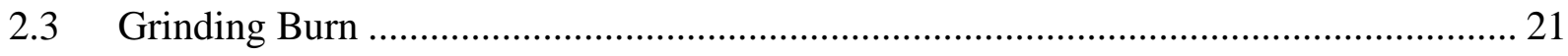

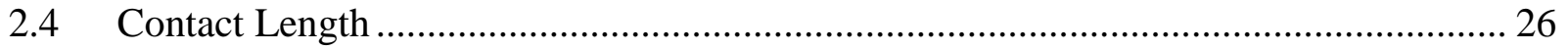

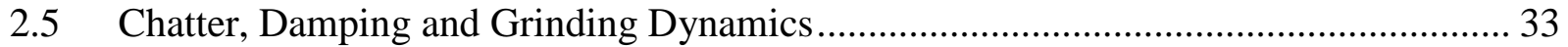

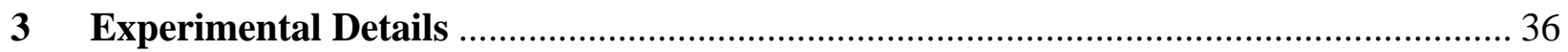

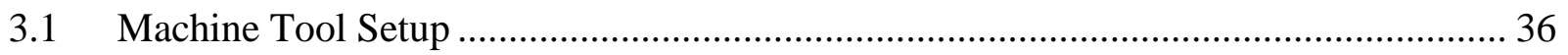

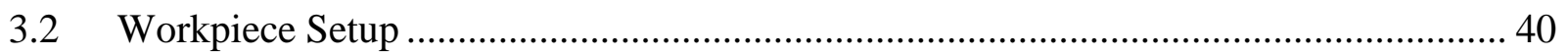

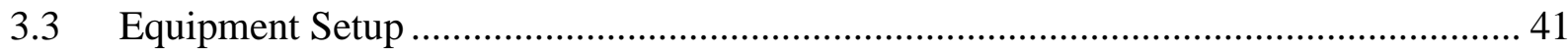

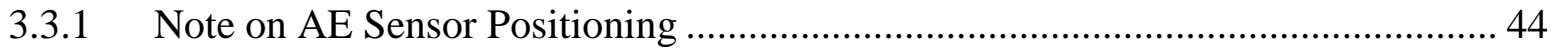

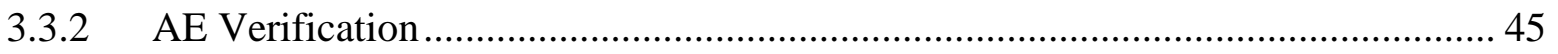




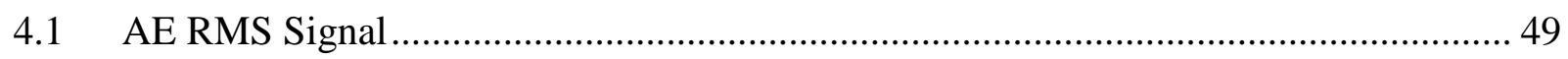

4.1.1 Effect of Successive Grinding Passes and Burr Formation ............................... 49

4.1.2 Effect of Grinding Direction ................................................................... 53

4.1.3 Effect of Coolant Application ..................................................................... 55

4.1.4 Effect of Workpiece Geometry .................................................................. 57

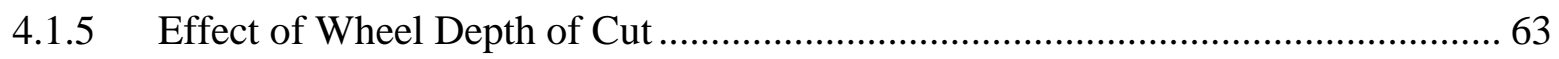

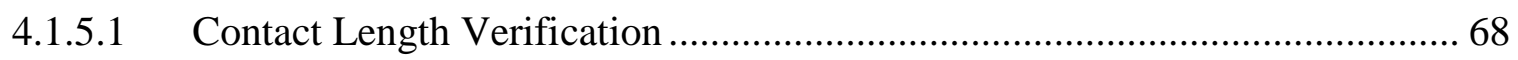

4.1.5.2 Effect of Workpiece Gap Separation....................................................... 75

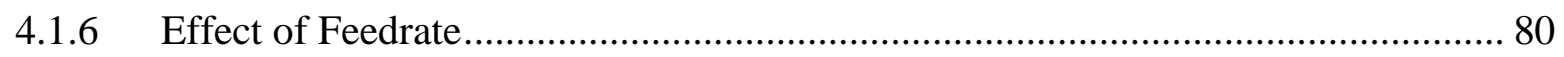

4.1.7 Effect of Grinding Wheel Speed................................................................... 82

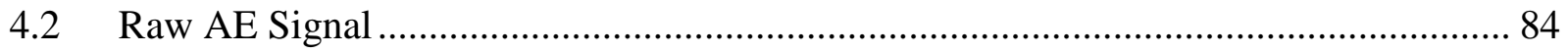

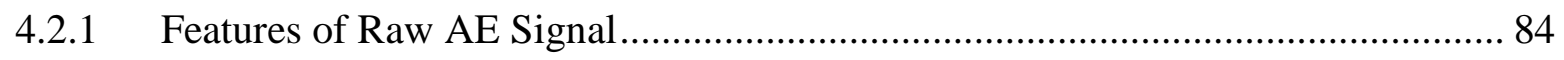

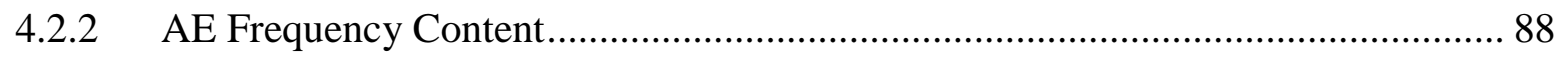

4.2.3 Grinding Burn and AE Frequency Content ................................................. 91

4.2.3.1 Why does Grinding Burn occur at Entry/Exit Region? ............................... 98

4.2.3.2 Distinguishing the Origin of AE Frequency Components........................... 103

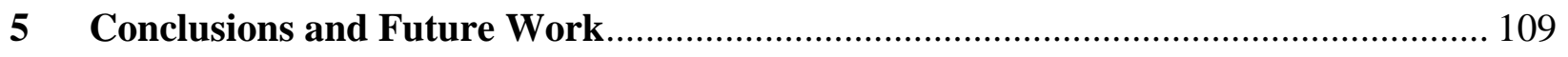

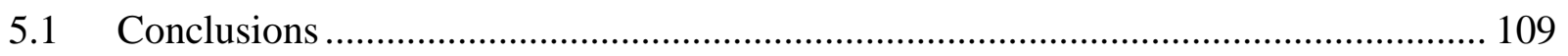

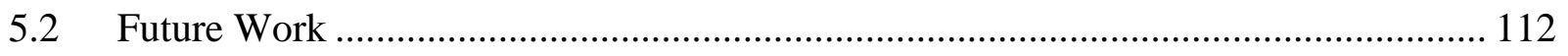




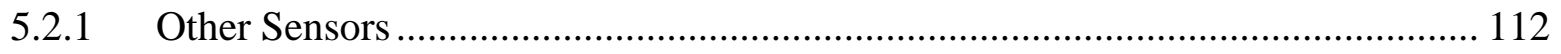

5.2.2 Workpiece Material ............................................................................. 114

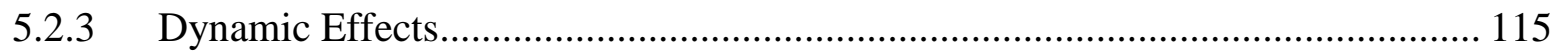

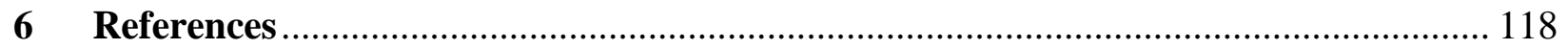




\section{List of Figures}

Figure 1.1: Illustration of the interaction between grinding grains and the workpiece [1] ........... 2

Figure 1.2: Comparison of up and down-grinding............................................................. 3

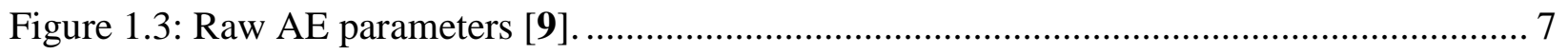

Figure 1.4: Common AE RMS signal observed during surface grinding.................................. 9

Figure 2.1: Typical raw AE signal during surface grinding [2] ........................................ 12

Figure 2.2: AE RMS calculated signal from a complete grinding pass, adapted from [2].......... 13

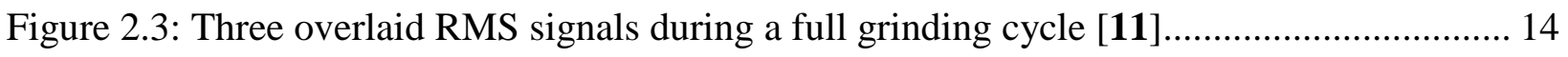

Figure 2.4: AE RMS response for a single chip formation in face milling [3]......................... 15

Figure 2.5: Raw AE signal when using a single tooth tool with a sharp cutting edge [4]........... 16

Figure 2.6: Spectral analysis of the continuous AE signal region in broaching ......................... 17

Figure 2.7: Burr shapes on the workpiece during surface grinding [18]. ............................... 18

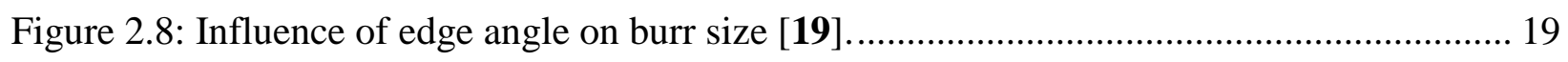

Figure 2.9: AE RMS amplitudes for burned and unburned components [2]........................... 22

Figure 2.10: Frequency content of a typical surface grinding pass [28]................................ 23

Figure 2.11: Illustration of cut in, steady state, and cut out regions for grinding [29]............... 24

Figure 2.12: Rowe's model of the grinding wheel and workpiece contact [34]....................... 27

Figure 2.13: Two-Half Slot grinding technique [36] ...................................................... 28

Figure 2.14: Peklenik method for measuring contact length [32]. ........................................ 29

Figure 2.15: Setup configuration of the thermocouple junctions [35].................................... 30

Figure 2.16: Contact length comparison using the Critical Contact State mode [32]................ 31

Figure 2.17: Contact length comparison using the Critical Contact State mode [36] ................ 31

Figure 2.18: Contact length results using the Applied Power Source method [35].................... 32 
Figure 2.19: Relationship between various grinding features and parameters. 34

Figure 3.1: BLOHM Planomat 408 surface grinder in the MSL of McMaster University. ......... 36

Figure 3.2: Relative tool path of an individual grinding pass............................................. 37

Figure 3.3: Workpiece engagement angles that define workpiece entry/exit geometry.............. 40

Figure 3.4: Frequency response of the AE sensor provided by the manufacturer [41] .............. 42

Figure 3.5: Experimental grinding setup with sensor positions........................................... 43

Figure 3.6: AE Raw and RMS signals of lead breaking using 1.2 ms time-constant [13]......... 45

Figure 3.7: RMS/Raw spike duration ratio as a function of RMS time-constant...................... 46

Figure 3.8: AE RMS entry and exit spikes are not significantly influenced by settling time ...... 47

Figure 4.1: AE RMS results from successive up-grinding passes (1-9) ................................ 50

Figure 4.2: Microscopic images of workpiece exit burr ...................................................... 51

Figure 4.3: Common AE RMS responses, comparing up-grinding to down-grinding................ 53

Figure 4.4: Illustration of the effect of workpiece entry and exit regions on burr formation ....... 54

Figure 4.5: AE RMS signals comparing the effect of coolant during up-grinding..................... 55

Figure 4.6: Comparison of AE RMS signals only changing workpiece edge geometry............. 58

Figure 4.7: Illustration of three different edge geometries using the same workpiece............... 59

Figure 4.8: Comparison of AE RMS signals only changing workpiece edge geometry............. 60

Figure 4.9: Factors contributing to the geometric contact length......................................... 64

Figure 4.10: AE RMS comparison at varying depths of cut................................................. 65

Figure 4.11: Comparison of an AE RMS entry spike and its derivative ................................. 66

Figure 4.12: Calculated wheel/work contact length vs. depth of cut for up-grinding. ................ 67

Figure 4.13: Calculated contact length vs. depth of cut for both up and down-grinding. ........... 69

Figure 4.14: Illustration of wheel/workpiece interaction at grinding wheel entry. ................... 70 
Figure 4.15: Geometric illustration of the grinding zone 71

Figure 4.16: The transition distance is dependent on workpiece geometry............................. 72

Figure 4.17: Results of AE RMS spike duration as function of workpiece geometry................ 73

Figure 4.18: Contact length measurement results obtained from RWTH Aachen University. .... 74

Figure 4.19: HSS workpiece with a physical gap introduced at its center. ............................. 75

Figure 4.20: Proposed principle of AE spike behavior at introduced gap ................................ 76

Figure 4.21: AE RMS results based on varying workpiece gap separation............................... 78

Figure 4.22: AE RMS signal comparison based on varying feedrate..................................... 80

Figure 4.23: Calculation of the contact length at varying feedrates. ...................................... 81

Figure 4.24: AE RMS signal comparison based on varying wheel speed................................. 82

Figure 4.25: Plot of raw acoustic emission data, with a post-processed RMS signal overlaid. ... 86

Figure 4.26: Raw AE signal representing initial grain contact during workpiece engagement. .. 87

Figure 4.27: Frequency content of the entire AE signal. .................................................. 88

Figure 4.28: Comparison of frequency content between AE signal regions. ............................ 89

Figure 4.29: Frequency comparison of grinding scenarios with burn and without burn [23]..... 90

Figure 4.30: Frequency content comparison of discrete AE signal regions. ............................ 92

Figure 4.31: Frequency response comparison based on a 140-160 kHz filtered signal. ............. 93

Figure 4.32: Illustration of sacrificial workpiece at workpiece entry region........................... 95

Figure 4.33: Filtered frequency results demonstrating the effect of a sacrificial workpiece........ 96

Figure 4.34: Illustration of workpiece edge geometry and bulk material................................ 99

Figure 4.35: Filtered AE RMS frequency component comparison. ..................................... 101

Figure 4.36: Illustration of different gap scenarios........................................................ 103

Figure 4.37: AE RMS filtered frequency components at the gap-region. ............................... 104 
Figure 4.38: AE RMS comparison of the three most dominant frequency components........... 106

Figure 5.1: Acceleration and normal force raw data........................................................ 113

Figure 5.2: Illustration of the setup problem involving static AE sensor positions................... 116 


\section{List of Tables}

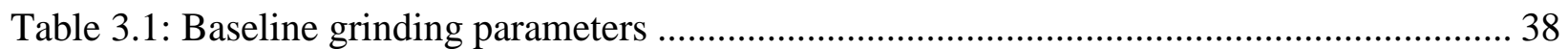

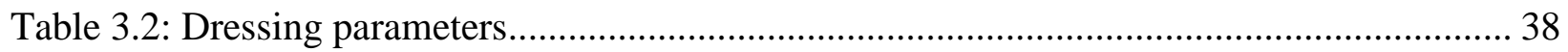

Table 4.1: Scenarios of AE spike existence and grinding burr formation ................................ 61

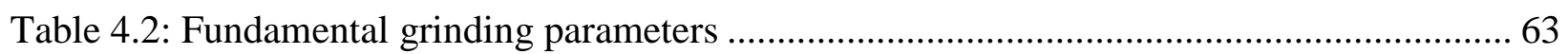


Dedicated to my loving family, who have always

supported me in everything that I do. 


\section{Introduction}

This chapter will present an overview of the components of this research project. A general introduction to surface grinding and acoustic emission follows, in terms of their significance to the scope of this work.

\subsection{Surface Grinding}

Grinding is an abrasive machining process that uses a rotating grinding wheel as the tool. It is commonly known for its ability to produce fine surfaces and accurate dimensions, but is also capable of removing large volumes of material quite rapidly as well. The most basic form of grinding which will be focused upon in this thesis is termed surface grinding, and is predominantly used to precisely finish flat surfaces. For this process, the machine tool typically consists of a magnetic reciprocating table that firmly holds the workpiece in place and a precision controlled grinding wheel. Surface grinders come in a variety of forms, from manually controlled machines, to fully programmable CNC configurations requiring very little operator involvement.

The grinding process can be largely compared to traditional metal cutting processes as individual grains that comprise the surface of the wheel act as microscopic single-point cutting edges. Tiny sheared chips are formed as these grains come into contact with the workpiece, similar to the much larger chips that are commonly produced in conventional machining. Figure 1.1 illustrates how abrasive grains on the wheel surface interact with the workpiece at the micro-scale. 

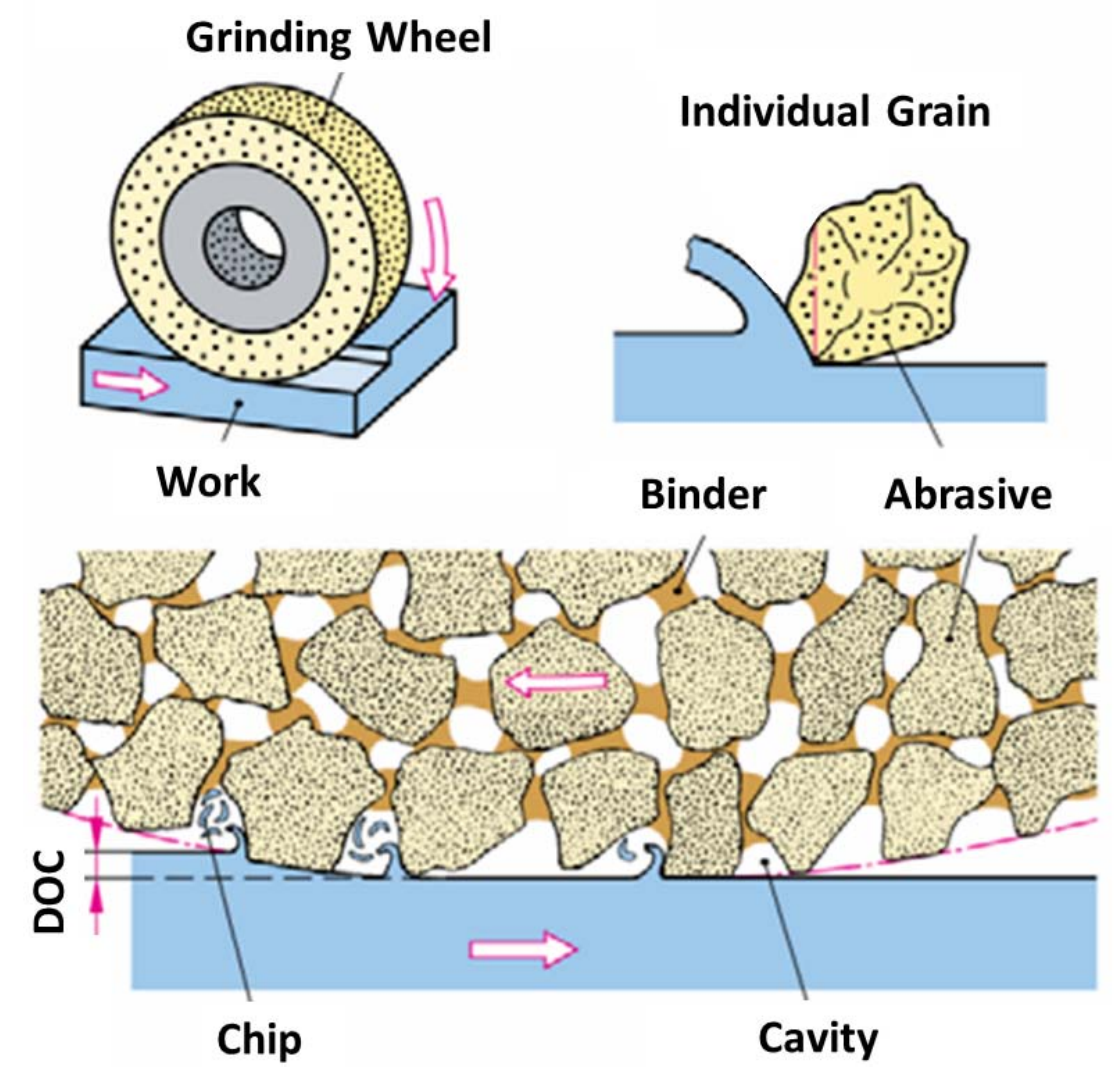

Figure 1.1: Illustration of the interaction between grinding grains and the workpiece [1].

The sense of scale must be clarified when observing the figure above. The diameter of a typical grinding wheel can range upwards of $300 \mathrm{~mm}$, while the wheel depth of cut (DOC illustrated here) is commonly on the order of tens of micrometers. This renders the chip removal process to be on the micro-scale, with hundreds of cutting edges engaged with the workpiece at any time. Similar to traditional cutting tools, these cutting edges can become dull during the course of machining. This is referred to as grinding wheel wear which affects performance. It can include wheel glazing through the formation of wear flats on individual abrasive grains, unintended grain fracture, or clogging of the open pores on the surface of the wheel. In order to remedy this problem before it begins to significantly affect grinding performance and surface quality, dressing techniques are implemented. 
Dressing refers to restoring the wheel to its original topography by dislodging worn abrasive particles to reveal the sharp grains underneath. The most common method is to use a diamond dressing tool that is traversed along the peripheral surface of the rotating grinding wheel. Due to diamond being the harder of the two materials, the exposed grinding grains are removed as they come into contact with the dressing tool. Grinding fluids can vary by type, but are commonly applied via high pressure nozzles directly into the grinding zone to lubricate, absorb heat, reduce wheel wear, and improve surface finish.

As the reciprocating table traverses back and forth during common surface grinding operations, the terms up-grinding and down-grinding become important to understand. Upgrinding is defined to occur when the tangential velocity of the grinding wheel is opposite to that of the workpiece velocity. Conversely, down-grinding occurs when both the directions of the workpiece and grinding wheel are the same. Figure 1.2 illustrates both up and down-grinding directions.

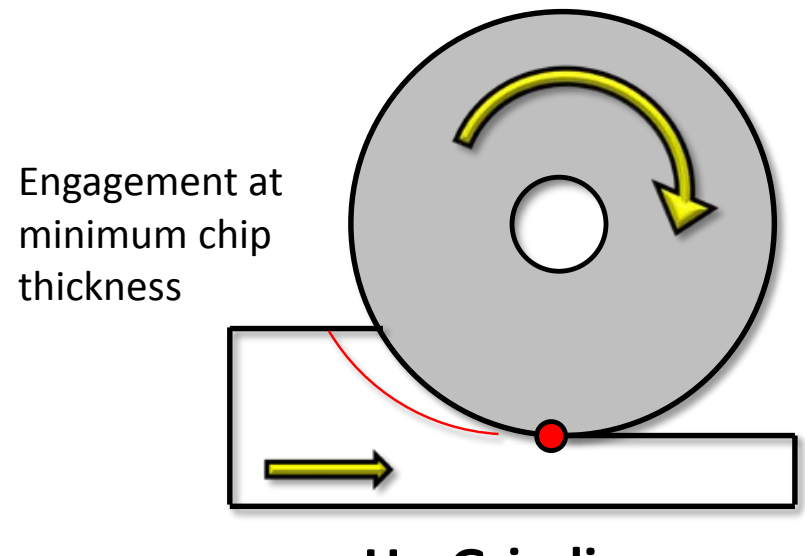

\section{Up-Grinding}

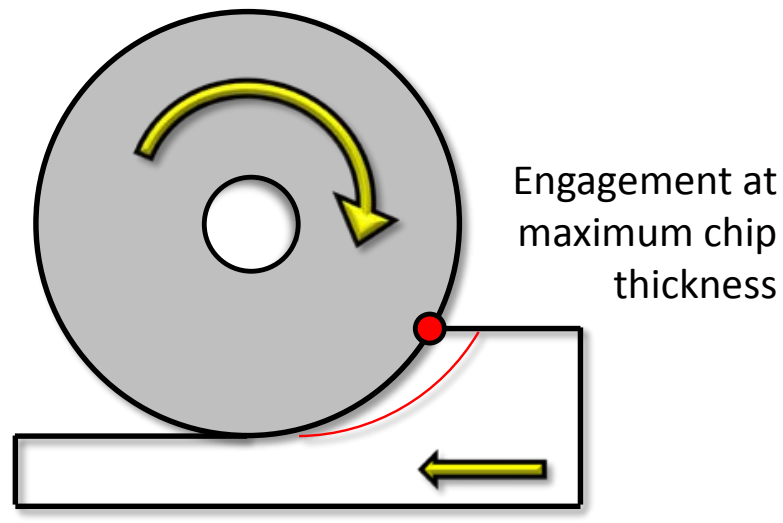

Down-Grinding

Figure 1.2: Comparison of up and down-grinding. Chip formation begins at its point of minimum thickness in the case of up-grinding, and begins at its point of maximum thickness in the case of down-grinding. Not to scale. 
There are significant differences in the material removal process that are a direct result of choosing either up-grinding or down-grinding. In the case of up-grinding, abrasive grains begin their cutting path from an initial chip thickness of zero and finish at the maximum chip thickness position. This trend is just the opposite in the case of down-grinding, whereby the abrasive grains enter the cut at the maximum chip thickness and exit at zero chip thickness.

One of the most notable differences between grinding and other metal cutting processes is that the position and geometry of each individual grain is probabilistic. Considering that grinding speeds are typically $30-60 \mathrm{~m} / \mathrm{s}$ or higher, and that the positions and morphology of grains are constantly changing due to wear and fracture, grinding processes are indeed complex. Monitoring the process is hence essential, especially when manufacturing valuable, high precision components.

One particular method of monitoring the grinding process involves acoustic emission, which will be detailed in the following section. Several recent publications report the occurrence of spikes in the acoustic signal at the workpiece entry and exit, but fail to clarify their origin $[2,3,4]$. Comprehension and potential application of this currently unexplained phenomenon comprises this thesis work. 


\subsection{Acoustic Emission}

There are several sensors currently being implemented in order to monitor the grinding process. Force, power, and acceleration sensors do provide useful information regarding the grinding system, but acoustic emission (AE) sensors have emerged to be one of the most suitable choices for grinding process monitoring [5]. Being able to detect workpiece contact, thermal effects, wheel wear, and machine chatter allows for greater process control. Process monitoring provides insight into the mechanical system, resulting in enhanced performance and improved quality.

Acoustic emissions are transient ultrasonic waves caused by rapid local stress redistributions [6]. AE is traditionally categorized as a non-destructive testing technique and can be implemented for either discrete testing or continuous monitoring. Surface-coupled piezoelectric sensors are most commonly used to obtain information regarding damage mechanisms, such as crack propagation, but they are far from being limited to this type of testing alone. AE sensors are ideal for machining process monitoring as they are extremely sensitive to the many dynamic interactions occurring between the tool and the workpiece.

One of the most notable differences between other sensor types and acoustic emission sensors is the operating frequency range. When comparing an $\mathrm{AE}$ sensor to a common accelerometer for example, the frequency ranges can differ by an entire order of magnitude. Whereas a standard accelerometer detects frequencies between $1 \mathrm{~Hz}-20 \mathrm{kHz}$, an $\mathrm{AE}$ sensor generally operates in the $50 \mathrm{kHz}-900 \mathrm{kHz}$ range. This leads into the main issue of acoustic emission signal processing: computer hardware and software must be adequate to handle sampling rates high enough to avoid aliasing the raw signal. 
Another notable detail is that acoustic emission sensors do not detect direct vibration. If an $\mathrm{AE}$ sensor were coupled to a vibrating object, its response would be generally unaffected. This is not to say that vibration cannot induce acoustic emission, but simply that most vibrations lie below the AE frequency range and go undetected [7].

The AE signal can be classified as either a continuous-type AE signal or a burst-type AE signal. In terms of machining process monitoring, continuous-type signals are generally associated with plastic deformation in ductile materials, while burst-type signals are more commonly observed during crack growth or fracture [8]. All of these detected AE events are added to give what is termed the raw AE signal.

\subsubsection{Raw Signal}

Acoustic emission generation is a transient phenomenon, meaning that acoustic events pass extremely quickly into and out of existence. The raw acoustic emission signal, when collected as a continuous waveform, is a compilation of all of the individual $\mathrm{AE}$ events that occur during the recorded time interval.

However, there are several $\mathrm{AE}$ features and parameters to recognize before simply collecting data. As a result of the random and rapidly changing AE signals, a way of differentiating useful data must be established. The number of times the signal crosses a set threshold is referred to as $\mathrm{AE}$ counts, and is sometimes used to quantify the overall $\mathrm{AE}$ activity of the system. 
Figure 1.3 illustrates some common terms associated with AE signals. The amplitude of the signal refers to the peak voltage of the response and is expressed on a relative decibel scale. Rise time is defined as the time between the first AE hit and the maximum peak amplitude, and the duration of the signal refers to the time interval between the first and last AE threshold crossing [9].

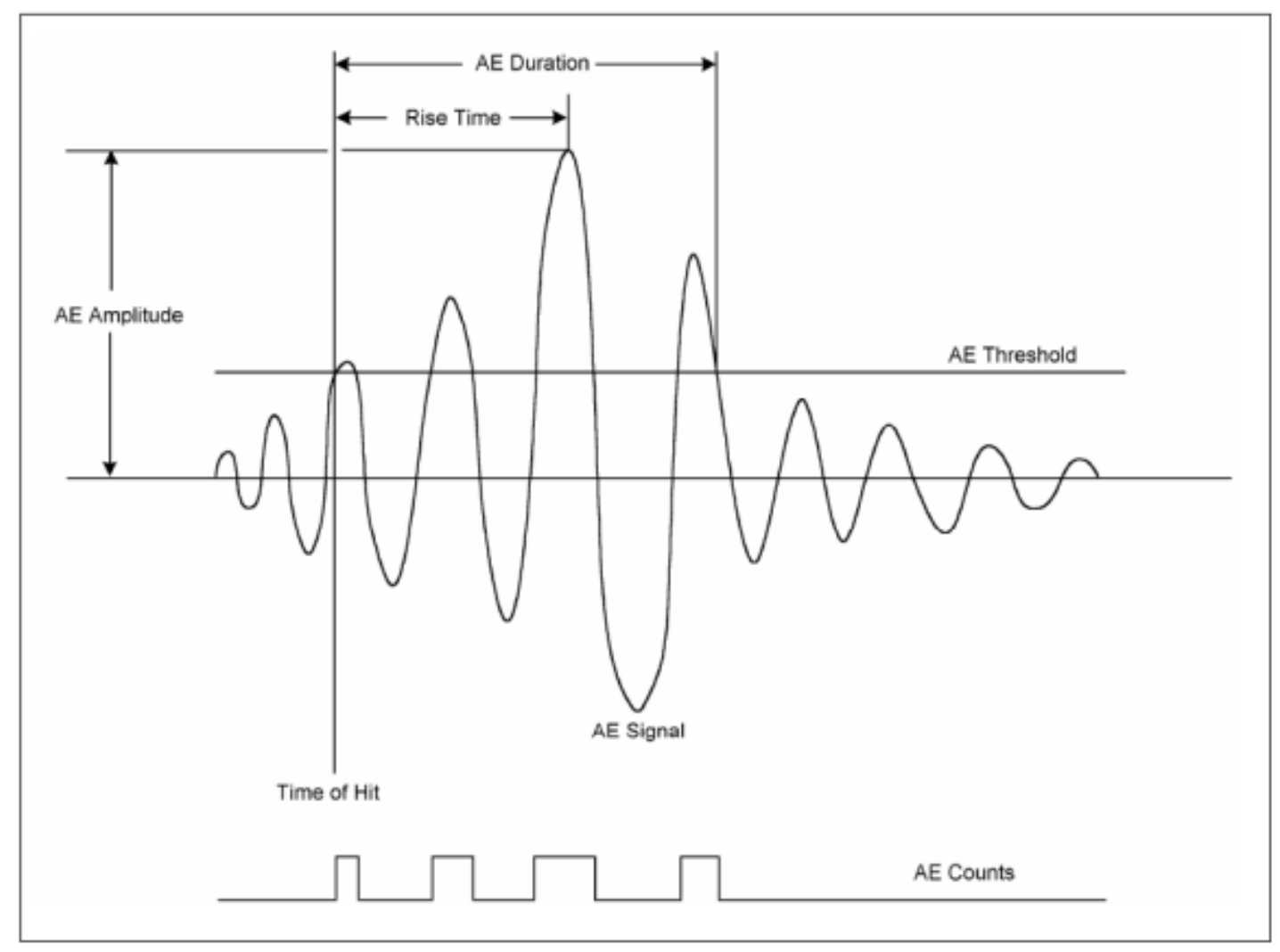

Figure 1.3: Raw AE parameters [9].

The raw signal proves to be very sensitive and can contain a significant amount of information regarding micro-scale events. Although AE research in grinding has been growing, there are a surprisingly low number of actual raw signal investigations. This is likely due to the complexity of the data collection process, which have led to the simpler time-averaged versions of the signal to be more commonplace. 


\subsubsection{RMS Signal}

The root mean square (RMS) provides a method of reporting continuously varying $\mathrm{AE}$ data in a much simpler way. It is defined as a rectified time-averaged value, calculated over a discrete interval, and is reported as a voltage in terms of $\mathrm{AE}$ sensor response. As soon as an $\mathrm{AE}$ hit is triggered, all of the features that make up the signal are 'averaged' over a predefined segment that is directly dependent on a chosen RMS time-constant. Typical AE RMS timeconstants range between 0.1 and 100 milliseconds [10] depending on the application, and can be implemented via analogue filters or through digital processing.

Although RMS signals do not provide as many of the individual details contained within the raw signal, its main advantage is the increased simplicity of its acquisition due to much lower sampling rates. The AE RMS signal is useful when monitoring varying amplitudes of the raw signal caused by unique events within the process. Although more economical in terms of viewing the AE data, its limitations must be understood to avoid making invalid assumptions. Frequency analysis of the raw signal is not possible if only the RMS data is collected, making it important to understand which signal is best suited to varying applications.

\subsection{Scope and Organization of Present Work}

Grinding has been an area of considerable research, but it still remains to be one of the least understood machining processes. With the increased industry reliance on CNC machining, it has become paramount that this process is carefully monitored. Simply monitoring the feedback signals from various sensors is indeed beneficial, but it is perhaps more important that the behavior of these signals and their fundamental causes are fully understood. Exploring a new phenomenon may not necessarily lead to an immediate or particular application, but by looking deeper into unexplained areas, new valuable methods can develop. 
One such phenomenon entails unexplained acoustic emission spikes that occur at the workpiece entry and exit during interrupted machining operations, most notably during surface grinding. These AE spikes are nothing new, as they have been reported to exist [2], yet they still remain to be explained in terms of their cause, or interpreted to yield useful information on the grinding process. A common AE RMS signal corresponding to a single grinding pass is presented in Figure 1.4.

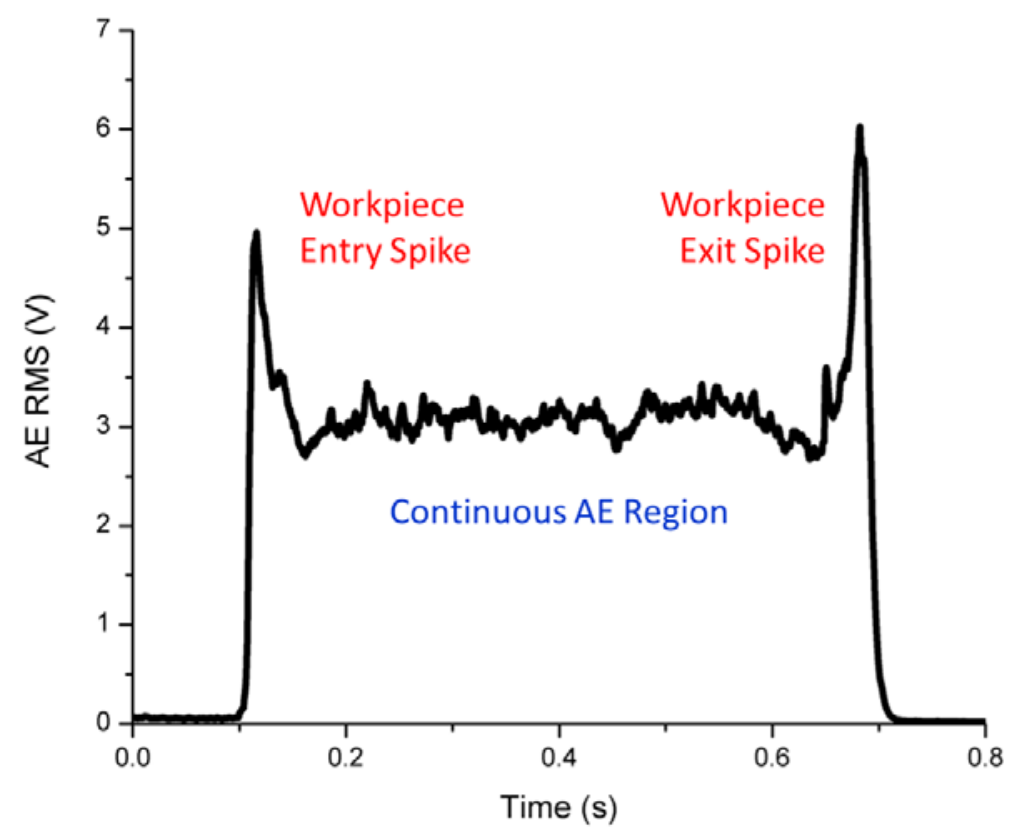

Figure 1.4: Common AE RMS signal observed during surface grinding.

This thesis work will hence focus on illuminating the physics referring to AE entry and exit spikes during surface grinding, which can be deemed to be a project that is purely curiosity driven. Experiments were carried out in order to determine the factors that influence the resulting $\mathrm{AE}$ spikes and to understand why they affect the signal so significantly at workpiece entry/exit. It was also a goal to determine if it was possible to understand the AE spikes in fundamental terms to provide beneficial information about the surface grinding process. 


\section{Review of the Literature}

To provide a background for the components of this work, a basic review of relevant literature is presented in this chapter. Information found here elaborates on the topics introduced in Chapter 1.

\subsection{AE Entry/Exit Spike Phenomenon during Grinding}

Grinding is commonly considered to be a finishing process in the manufacture of many components requiring high tolerances and fine surfaces. With the continued industrial emphasis on increased productivity and quality, real-time monitoring of the grinding process has been an area of considerable research. Due to the numerous and rapid interactions between the surface of the grinding wheel and the workpiece, acoustic emission has been recognized as one of the leading techniques for grinding process monitoring. Hundt et al. [5] states that AE signals contain "all of the information of interest about the micromechanical phenomena of the grinding process” and that they are far better suited for monitoring very fast events compared to traditional force measurements. Accordingly, studies have been conducted that utilize AE signal data to sense wheel wear, thermal damage, and contact detection during grinding $[\mathbf{8 , 1 0 , 1 1 ]}$.

However, typical acoustic emission monitoring refers to the RMS calculated response as it is far less intensive to implement compared to the raw signal. Webster [10] notes that the $\mathrm{AE}$ RMS signal may however leave many of the important instantaneous grinding features unidentified; this includes the "dynamic geometric error of the workpiece and the wheel, grinding burn and grinding cracks." He continues to state that a "fundamental study of the characteristics of the raw AE signal is very important” in terms of grinding process monitoring, but has this been a lacking area of research. 
Notwithstanding the lack of raw signal analysis, the much simpler RMS signals are frequently misinterpreted as well. The most significant factor when working with the RMS signal is the time-constant. Equation 2.1 governs the RMS value, where $\mathrm{N}$ is the discrete number of $\mathrm{AE}$ data points within the integration time-constant $\mathrm{T}$ [12].

$$
A E_{R M S}=\sqrt{\frac{1}{T} \int_{t-T}^{t} A E_{r a w}^{2}(\tau) d \tau} \approx \sqrt{\frac{1}{N} \sum_{i=1}^{N} A E_{\text {raw }}^{2}(i)}
$$

As the time-constant is increased, the length of the RMS averaged segment increases. This provides a cleaner signal, but will eventually filter out amplitude variation as $\mathrm{T}$ is continually increased. If the time-constant is set to the length of the entire AE signal, this refers to a single RMS value. Considering the opposite extreme, whereby the time-constant approaches zero, the RMS signal will mirror the raw AE signal, albeit only displayed on the positive Y axis.

In several works, the AE RMS time-constant is either arbitrarily selected or not reported $[\mathbf{1 0 , 1 1}]$. This is of concern as the corresponding AE RMS signals being analyzed may become completely misleading from what they are expected to represent, which is the raw signal.

Webster's work [10] outlines the initial role of the time-constant, showing its effect on the processed signal. His main finding is that the selected "integration time-constant has significant influence on the rise time” of the RMS response, with larger values of T resulting in a longer rise time. He concluded that in terms of the grinding process, a time-constant of $1 \mathrm{~ms}$ is recommended to appropriately represent the true raw $\mathrm{AE}$ signal. A more recent study by Jemielniak [13] was based on Webster's findings, but reported that "if an AE RMS signal is to be used for AE burst analysis in cutting, the integration time-constant should not exceed about 
0.1 ms." This discrepancy is important to understand in terms of this thesis research, and is addressed in Section 3.3.2.

There are very few actual reports on the raw acoustic emission signal during grinding, which is of concern as most works discuss RMS values without any basis for comparison. Webster's work is among the very few that include a typical raw AE signal that refers to a surface grinding pass, shown in Figure 2.1.

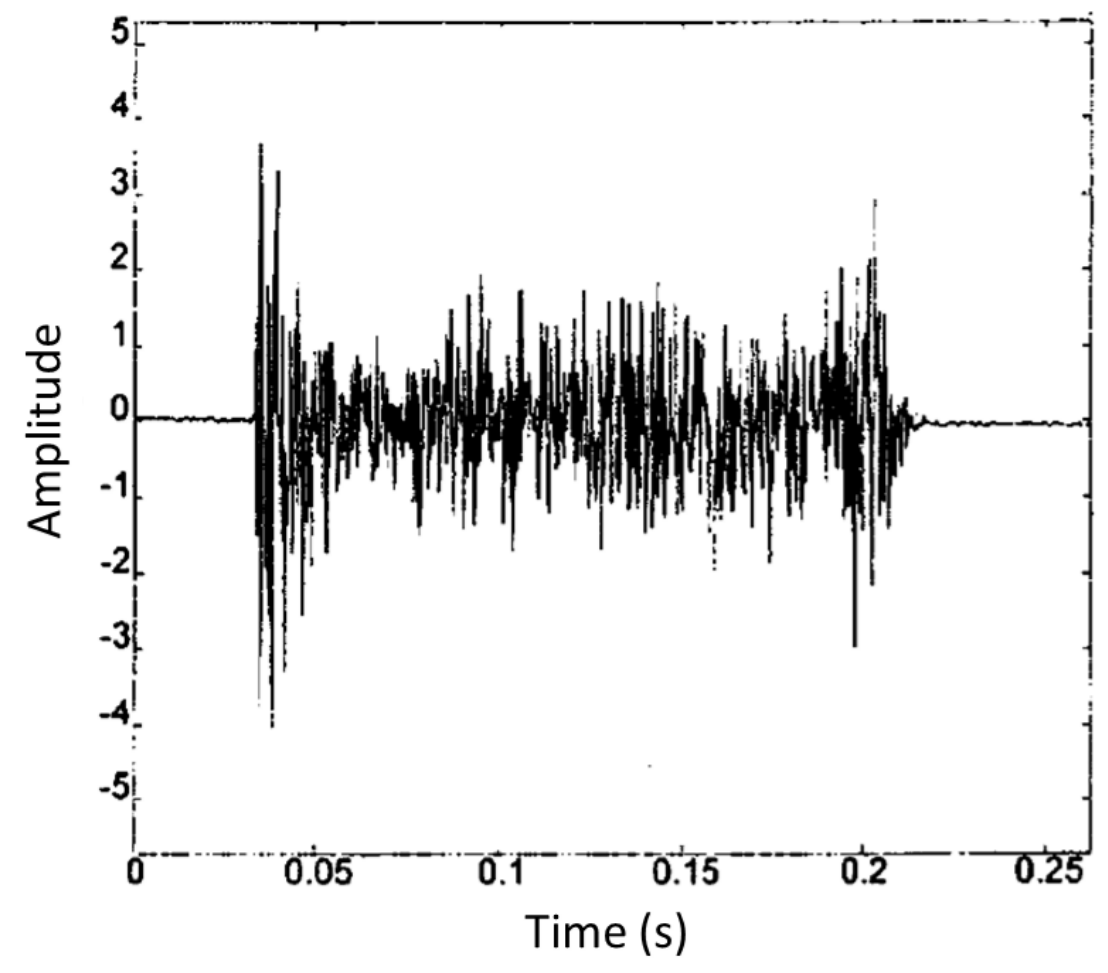

Figure 2.1: Typical raw AE signal during surface grinding [2].

In 1994, this work was quite remarkable, as the acquisition process results in a "huge amount of data, which soon fills a hard drive, leading most researchers to concentrate on the AE RMS filtered signal” [2]. Sampled at a frequency of $2 \mathrm{MHz}$, Webster's raw AE data clearly shows the phenomenon of signal 'spikes' occurring at workpiece entry and exit. He proceeded 
to digitally calculate the AE RMS signal from the raw data, yielding the result shown in Figure 2.2.

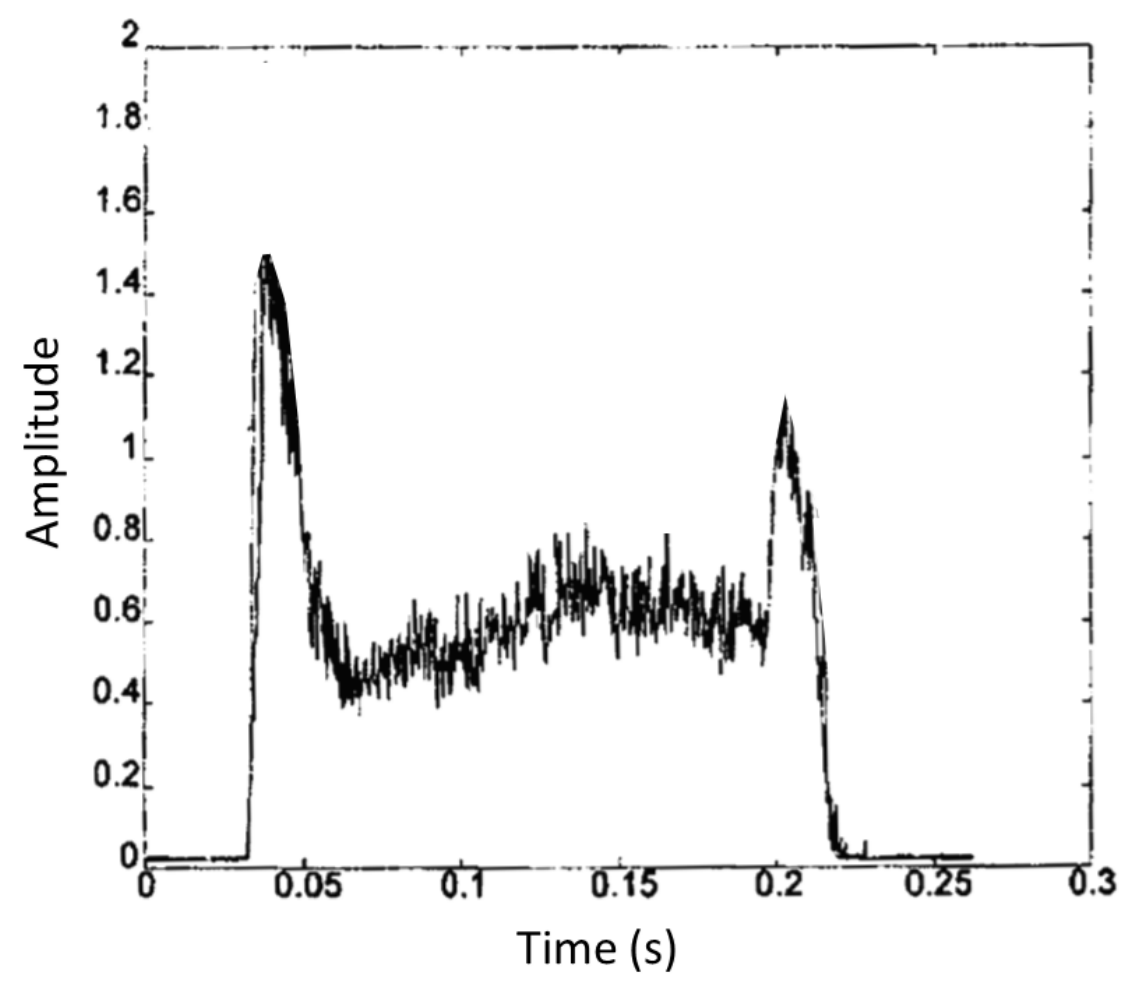

Figure 2.2: AE RMS calculated signal from a complete grinding pass, adapted from [2].

Webster's result shows that "the largest AE RMS amplitudes occur at the edges of the workpiece, which is an interesting characteristic of AE” [2]. This phenomenon is the basis for this thesis work, as it is not understood. Commonly dismissed as "an impulsive random signal that enters the workpiece at the contact zone,” or blamed on signal distortion, the AE spikes have actually been digitally filtered out in some cases with "means of recovering a signal which has been substantially altered in transmission” [2].

However, putting aside the unexplainable AE entry and exit spikes for now, a much more pressing issue relates to the general RMS analysis. Webster's result shown in Figure 2.2 accurately represents the changes in amplitude of the raw AE signal, as the RMS time-constant 
was properly selected. When comparing the results to other research, the AE RMS signals during similar grinding experiments show very minimal entry and exit spikes. Figure 2.3 shows the AE RMS signals reported by Hassui et al. [11] wherein the spikes are not as significant as seen in Webster's work.

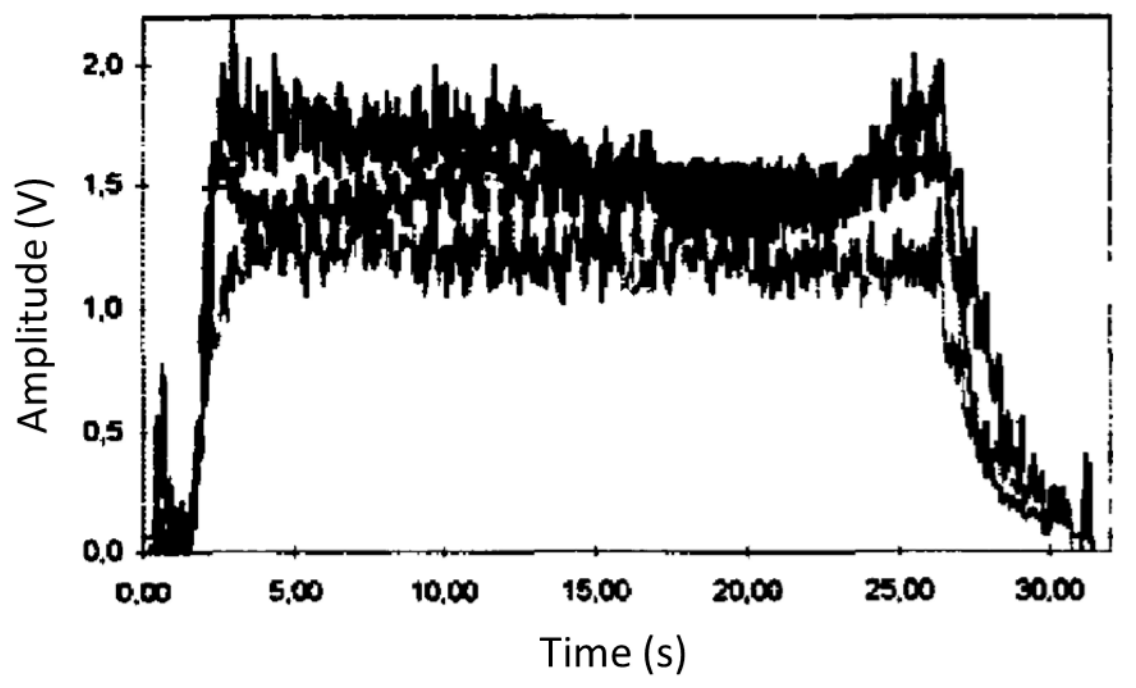

Figure 2.3: Three overlaid RMS signals during a full grinding cycle all showing a similar response [11].

Since the actual raw AE signal was not reported in [11], it is difficult to verify if the calculated RMS response is accurate. It is of concern that this method of AE analysis has almost become accepted, without understanding that the raw signal must first be considered. In Figure 2.3, the time-constants used for basing this result on were set to $2 \mathrm{~ms}$ and $37 \mathrm{~ms}$. With values this high, any amplitude spikes would be acutely averaged-out, yielding an RMS signal that may be completely misleading. Explaining the AE entry and exit spike phenomenon during grinding is perhaps not as important as being aware of proper data analysis techniques. The consequences of using an arbitrary RMS time-constant value must be understood before examining the resulting signal. This deceptive RMS signal may be responsible for the very low number of reports that identify how significant AE RMS entry/exit spikes can be during 
grinding. As a result of this, literature referring to other machining processes had to be considered to gain better insight into AE spike phenomenon.

\subsubsection{AE Entry/Exit Spike Phenomenon during Cutting}

Upon further investigation of reported AE entry/exit spikes, it was found that they are a common feature in many interrupted cutting processes as well, in addition to grinding. Jemielniak reported that AE "bursts can be generated due to tool engagement and disengagement with the workpiece" during interrupted turning "which can conceal useful signals normally generated by the tool breakage” [14]. In this case, the entry/exit phenomenon is not understood, and is instead disregarded in an attempt to focus on only the AE spikes associated with catastrophic tool failure.

Similar reports are documented during face milling [15], whereby "the discontinuous nature of the cutting introduces two additional sources of $\mathrm{AE}$ at the tool entry into and exit from the workpiece." Dornfeld's work is referenced in Figure 2.4; in which a "significant increase in $\mathrm{AE}$ signal upon the initiation of the chip formation (due to initial impact of the insert with the workpiece) and exit of the insert from the workpiece” has been observed [3].

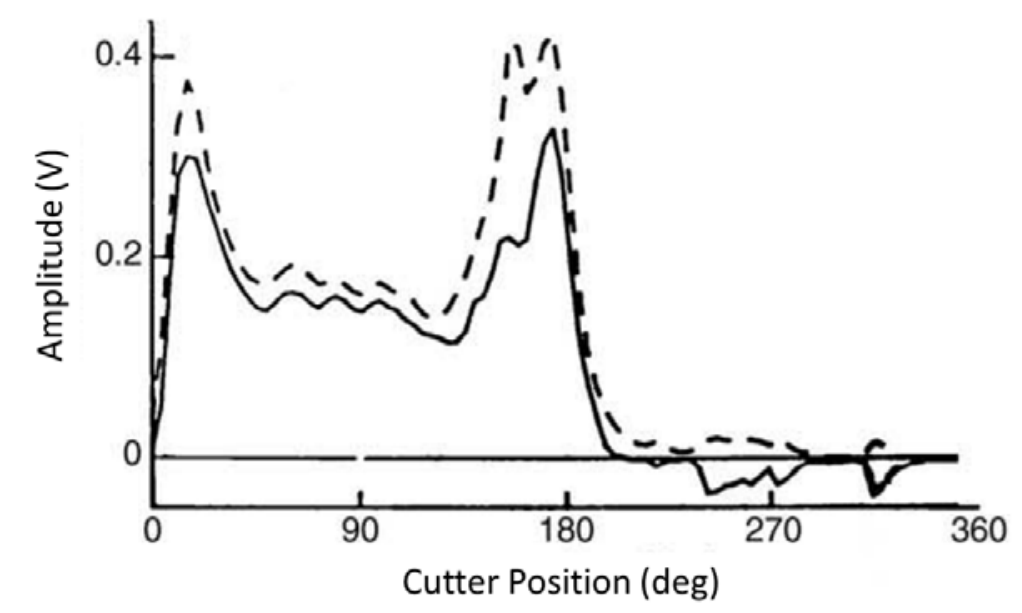

Figure 2.4: AE RMS response for a single chip formation in face milling [3]. 
The most common conclusion of the existence of AE spikes in these reports is to do with the tool impact with the workpiece. However, it is rare to find any elaboration on this, with most works simply mentioning the phenomenon as opposed to an explanation of their occurrence. Axinte's work [4] is interesting in this regard, as he reports similar AE entry/exit signal bursts during broaching, but includes an analysis of the raw signal during interrupted cutting as well. Also provided in his work are possible reasons for these acoustic events: including damping, vibration, chatter and even burr formation.

Figure 2.5 shows the raw AE burst signals corresponding to tool entry and exit during broaching. This signal represents the removal of a single continuous chip. Axinte states that it is likely that the burst signal at the start of the cutting process is related to the start of plastic deformation occurring at the beginning of chip formation, and the burst signal at the end of the process is related with chip disengagement from the workpiece leading to burr formation [4]. Broaching is a very different machining process when compared to surface grinding; however, the raw AE signal presented here can be seen to generally behave similar to that shown in Figure 2.1.

Tool entrance

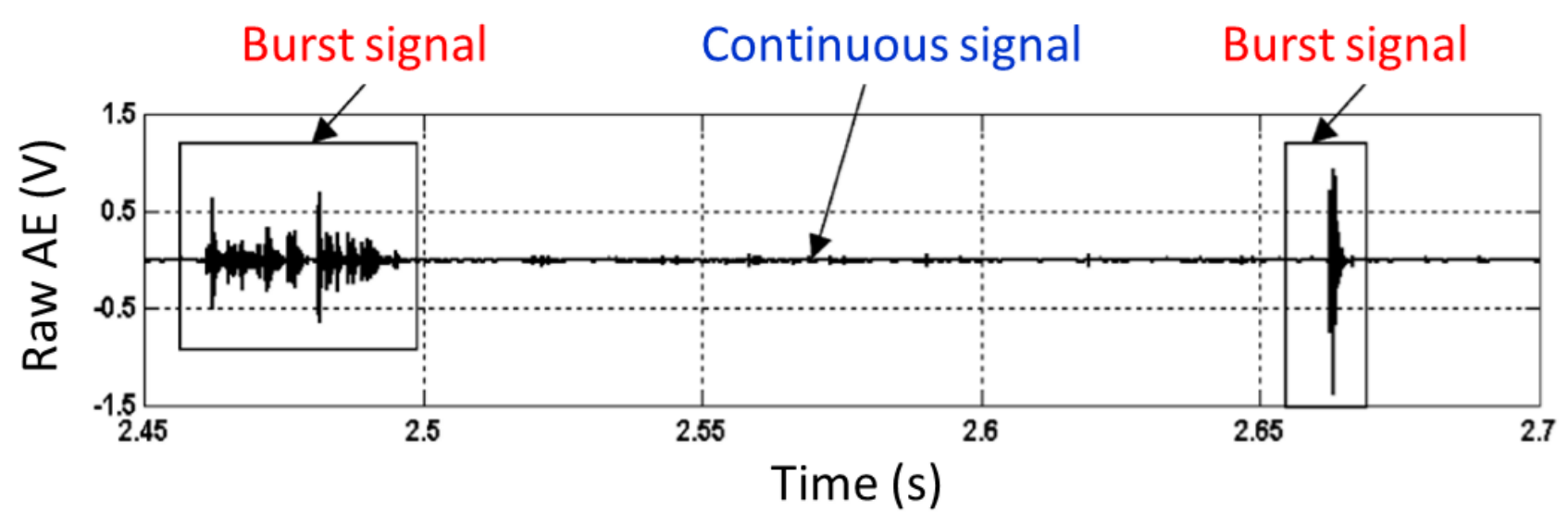

Figure 2.5: Raw AE signal when using a single tooth tool with a sharp cutting edge [4]. 
Additional work was performed by Axinte [16] regarding a frequency analysis of the raw AE during broaching and is presented in Figure 2.6. Note that the dominant frequency bands are on the low end of the typical AE spectrum, close to the $100 \mathrm{kHz}$ range. This will be compared to grinding AE frequency content in Section 2.4, as it relates to thermal effects.
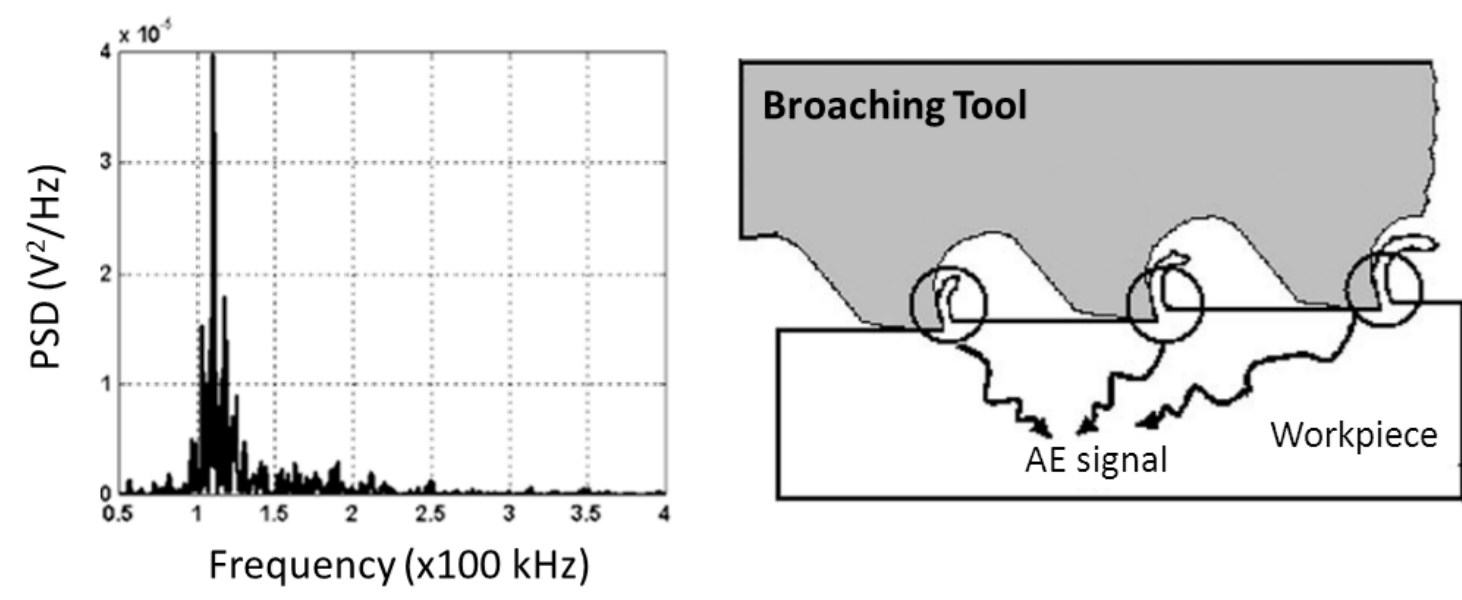

Figure 2.6: Spectral analysis of the continuous AE signal region with three broaching teeth cutting simultaneously [16].

Although the findings presented by Axinte are strictly based on broaching, he introduces several interesting ideas that may translate to the AE signal behavior during surface grinding. Concepts of system excitation "by entrances/exits of cutting teeth into/from the workpiece...that result in damped vibrations" [17] as well as the occurrence of chatter may also be relevant to the grinding process. Furthermore, Axinte’s earlier mentioned remark [4] regarding AE burst events and burr formation is worth further investigation. Grinding burrs at workpiece entry and exit can become quite significant, and may have an effect on the corresponding AE signal. The following section will hence discuss literature pertaining to the growth and influence of workpiece burrs during grinding. 


\subsection{Grinding Burrs}

There are several definitions of burr formation in the literature: from an "undesirable projection of material," to an edge or surface "which lies outside the desired geometry" [18]. In most cases however, "burrs are defined as the unwanted projections of material that form as the result of the plastic flow from cutting and shearing operations” [18]. In terms of the surface grinding operation, burrs are classified as either entrance burrs, side burrs or exit burrs and are located at the edges of the workpiece as illustrated in Figure 2.7.
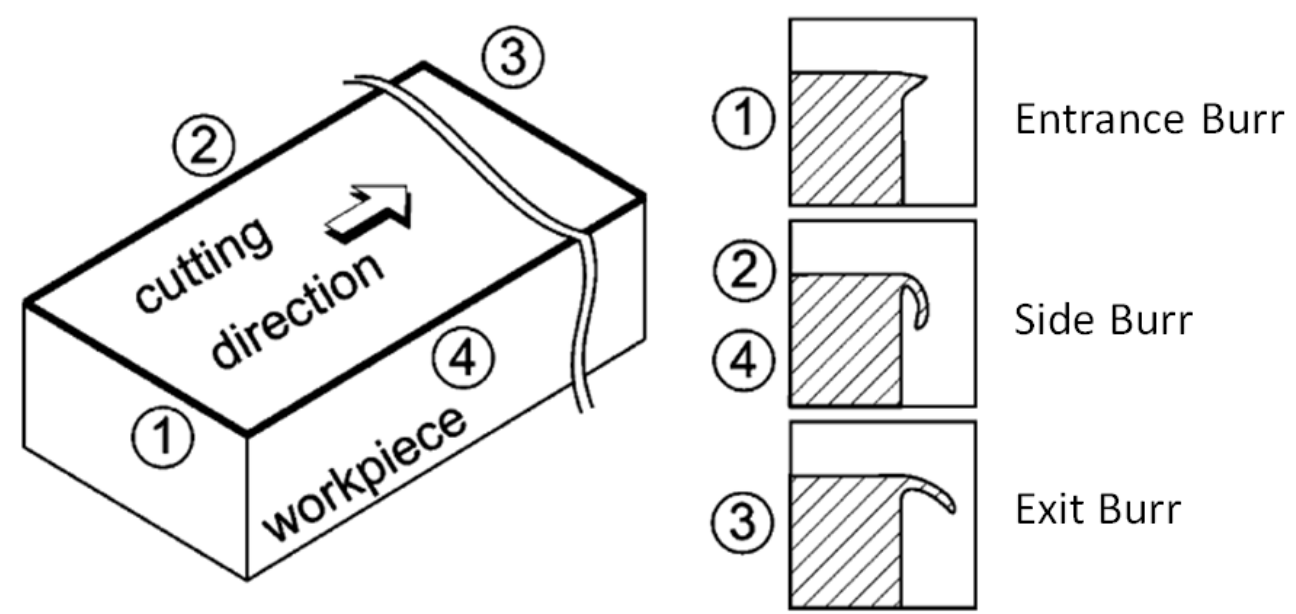

Figure 2.7: Burr shapes on the workpiece during surface grinding [18]. Note that exit burrs are generally more dominant than entry burrs.

Grinding burr formation is of particular interest as the associated plastic deformation is a known source of acoustic emission within the workpiece material. Reports that "a burr always forms if the material escapes the cutting process and occurs at tool entry and exit" [18] are of significance, especially when considering the AE entry/exit spike phenomenon that occurs in the same location. It is also of interest that burr formation in grinding is dependent on the entry/exit geometry, becoming "lower if the material is restricted to deform due to workpiece geometry conditions” [18]. 
Kawamura et al. [19] investigated burr behavior in grinding as a function of the workpiece edge geometry. Results from their work are shown in Figure 2.8, with reference to burr height and root thickness. It was reported that there was a tendency for the burr size to rapidly decrease as the edge angle becomes greater than $90^{\circ}$ as the expansion of the plastic zone is prevented [19]. This result was also supported by Barth et al. [20], with the explanation that "the edge of the workpiece does not offer sufficient resistance against plastic deformation."
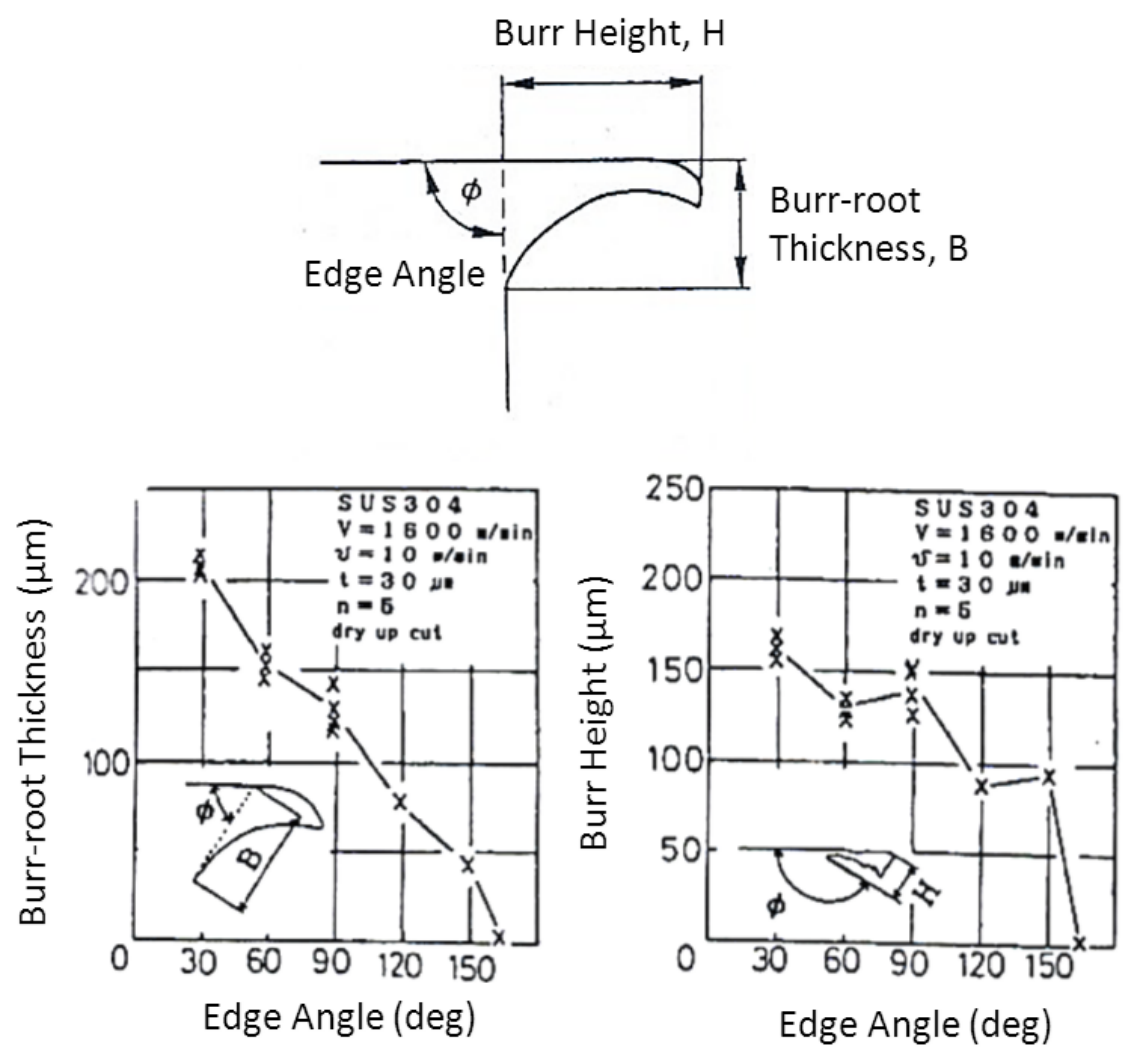

Figure 2.8: Influence of edge angle on burr size [19].

Increased plastic deformation at the workpiece edges due to grinding burr formation may be a contributing factor to corresponding $\mathrm{AE}$ entry/exit spikes as proposed by Axinte [4]. However, the formation of grinding burrs also effects thermal variations within the workpiece and is worth investigating in addition to plastic deformation. 
Aurich et al. [21] found that the temperature within the burr can strongly increase during its short grinding time, occasionally leading to "martensite hardening generated by a fast cooling rate." Van Behemen et al. [22] discussed the possibility in which "dislocation motion can be regarded as the origin of acoustic wave generation during martensitic transformation,” reporting it as a detectable source of $\mathrm{AE}$ within the material. This may perhaps contribute to entry/exit spike amplitude as grinding zone temperatures can reach levels in excess of $800^{\circ} \mathrm{C}$, well above the workpiece austenization temperature [21].

Returning to Kawamura's [19] work which found that burr size increases as the edge angle decreases, a relevant report by Barth [20] found that the workpiece temperature behaved in the same way. He found that the local temperature rise is higher when the edge angle is reduced "as the amount of heat-absorbing material decreases when the grinding wheel approaches the edge of the workpiece". A conclusion was made relating burr formation to "the material behavior of the workpiece which is a function of temperature" [20]. Aurich's work [21] agrees with these statements, finding that "the reduced surrounding material volume leads to a local temperature increase” at the instances of workpiece entry and exit.

With an understanding of how workpiece geometry influences both burr formation and the thermal effects during grinding, its influence on the AE entry/exit phenomenon will be an area of investigation in Chapter 4. With the gained knowledge of elevated workpiece edge temperatures, a relevant publication by Liu et al. [23] found that "thermal expansion accompanied by grinding burn triggers acoustic waves.” This indicates that grinding burn may also contribute to the $\mathrm{AE}$ spikes at workpiece edges, and so the following section will introduce its significance. 


\subsection{Grinding Burn}

Grinding requires a very large amount of energy to remove a unit volume of the workpiece, with almost all of it being dissipated as heat $[\mathbf{2 4 , 2 5 ]}$. Consequent high temperatures within the grinding zone can cause workpiece burn, phase transformations, surface softening and residual tensile stresses. Malkin [24] reports that "thermal damage is one of the main factors which affects workpiece quality and limits production rates” during grinding, and so its avoidance becomes critical during industrial applications.

Workpiece burn is the most common form of thermal damage and is typically the most undesirable. It can significantly reduce the fatigue life of the component, resulting in the mechanical integrity of ground parts to become compromised. Grinding burn is generally identified by visible changes in surface colour due to oxide-layer formation and has been found to be accompanied by workpiece reaustinization [24]. However, this visual method of classification becomes unreliable and difficult to standardize in terms of the degree of thermal damage being experienced. "The most accurate way to detect grinding burn is by examining the metallographic picture of the ground surface," but this is a very expensive and time-consuming task as it relies on destructive testing [23]. Therefore, the implementation of online monitoring to detect workpiece burn has become a recent area of investigation, with particular interest in acoustic emission techniques.

Eda et al. [26] studied the behavior of AE signals corresponding to varying levels of visual grinding burn, noting a general rise in signal amplitude with the development of workpiece burn. Webster's work [2]was found to support this result, as he reported that "burned workpieces have a higher RMS amplitude than unburned ones” as shown in Figure 2.9. 


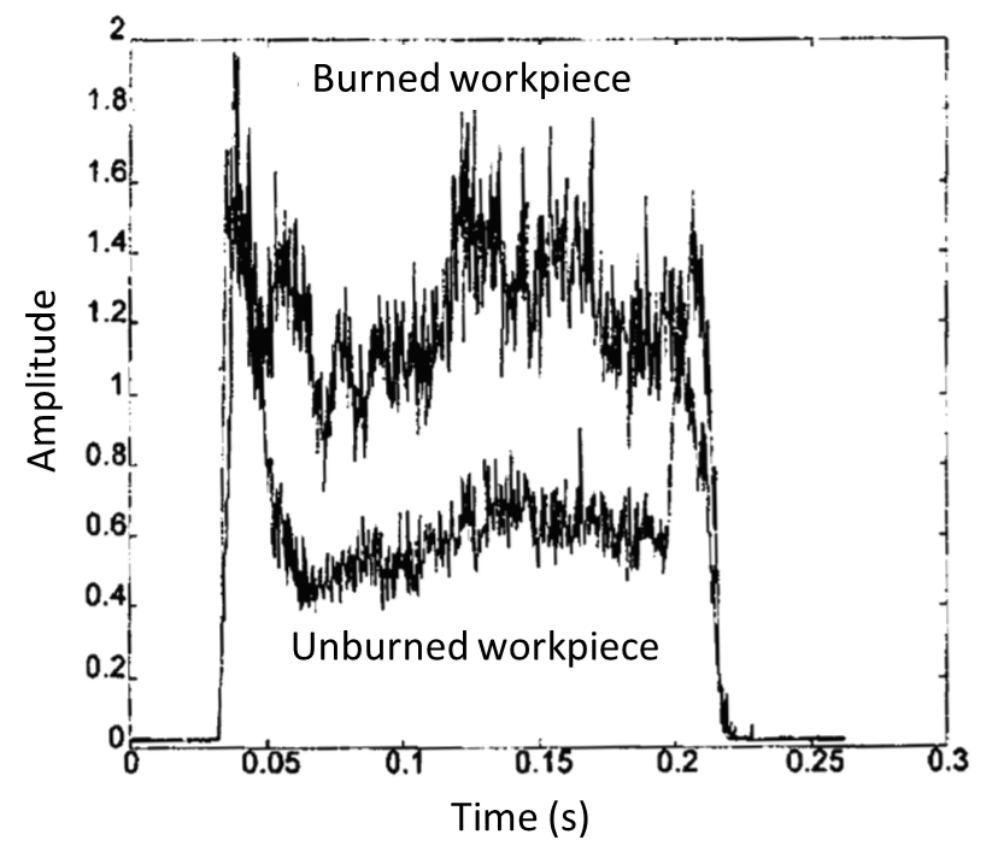

Figure 2.9: AE RMS amplitudes for burned and unburned components [2].

Eda reported that under constant grinding conditions, there is a possibility of detecting burn by utilizing the relative changes in signal level [26]. Although this method of analysis showed promise, it was primarily based on initial establishment of individual AE threshold levels corresponding to the level of thermal damage for every set of grinding conditions. This proposed method can soon become laborious, however, it must be recognized that this work was published in 1984, making it one of the earliest documentations of this phenomenon.

In addition to the overall $\mathrm{AE}$ response trends, Eda's work was more remarkably successful in investigating sections of the raw AE spectrum. Although digital processing was completed using less sophisticated equipment than is commonly used today, he was able to conclude that "frequency components of AE over $100 \mathrm{kHz}$ increase with the generation and progression of grinding burn” [26]. This is of particular interest as it was not until approximately 10 years later that Konig et al. [27] reported a similar result that "grinding burn can be detected 
in the selected frequency band (200-289 kHz)." It was also reported in this work that "RMS signals fail to supply a direct indication of grinding burn,” suggesting that raw AE frequency components are best used for monitoring thermal phenomena [27].

Figure 2.10 shows the typical AE frequency content of a surface grinding pass reported by Griffin et al. [28]. This result is of particular interest when compared to the AE frequency data during broaching presented earlier in Figure 2.6. Both sets of data show a dominant frequency component in the $100 \mathrm{kHz}$ range, however there is an additional higher frequency component observed during grinding just past $400 \mathrm{kHz}$.

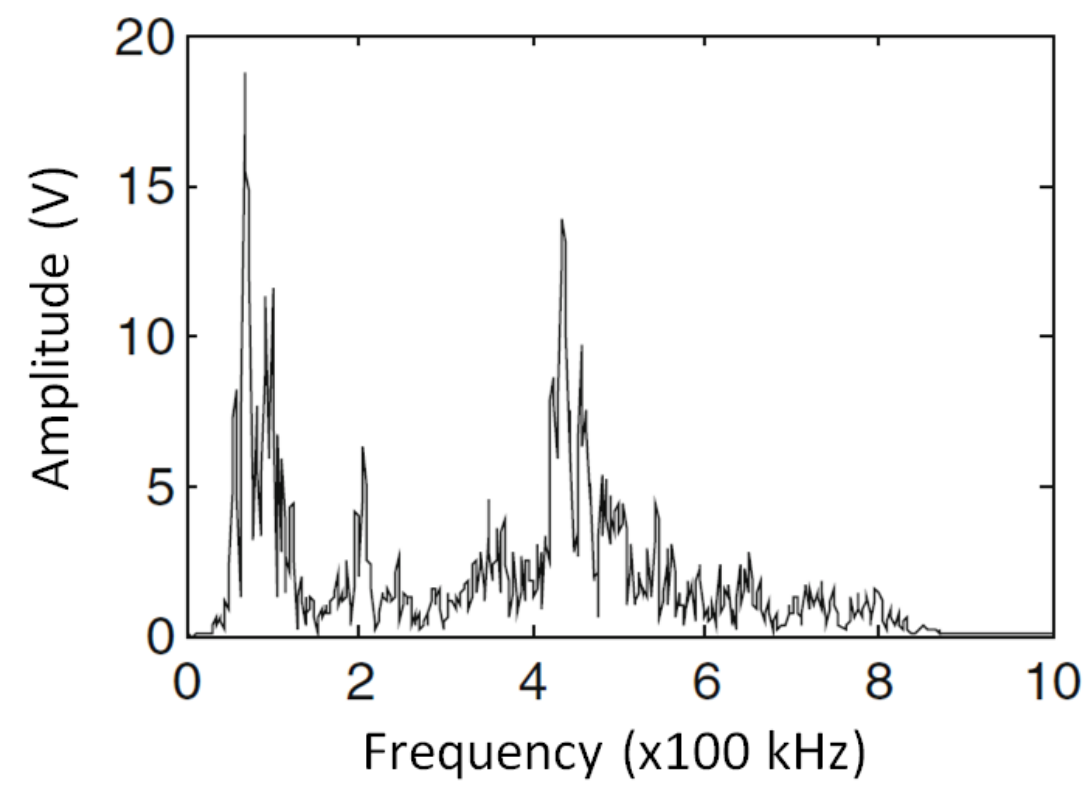

Figure 2.10: Frequency content of a typical surface grinding pass [28].

Solely considering thermal effects, high workpiece temperatures are relatively insignificant during low speed broaching, but well pronounced during grinding. If the higher frequency component is indeed related to thermal effects, it lends credence to both Eda's [26] and Konig's [27] earlier findings. This may suggest that there are separate AE events 
responsible for individual frequency components during machining, with those greater than $100 \mathrm{kHz}$ relating to an increase in thermal effects—-typical to grinding.

Online detection of grinding burn using acoustic emission has been discussed in $[2,23,26]$, but it remains an area of complexity as it is process specific. An alternative to this method comes via thermal modeling, and is a more common technique to control and prevent thermal damage from occurring in the first place. Several comprehensive studies regarding workpiece temperature simulations have been reported and experimentally tested, all yielding similar conclusions.

Temperature analysis during surface grinding is highly dependent on transient effects [29]. In cases where "the workpiece is long enough for the temperature within the grinding zone to reach a quasi-steady state...transient temperatures occur as the grinding wheel engages and disengages from the workpiece” [24]. These workpieces are 'geometrically long' if their length exceeds the wheel-workpiece contact length at the full depth of cut [24]. For this grinding scenario there are three distinct regions which can be considered; cut in, steady state, and cut out $[24,25,29]$. Each of these regimes correspond to unique thermal behavior, and are illustrated in Figure 2.11.

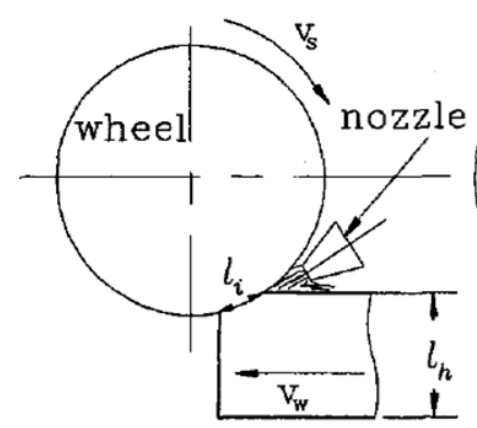

cut in

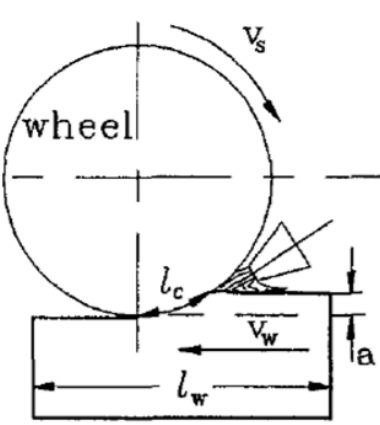

steady state

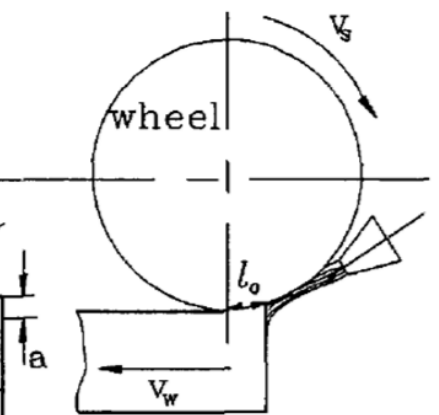

cut out

Figure 2.11: Illustration of cut in, steady state, and cut out regions for grinding a geometrically long workpiece [29]. 
It has been reported that "the temperature rises rapidly during initial wheel-workpiece engagement (cut in), reaches a quasi-steady state value only if the workpiece is sufficiently long, and increases still further during final wheel-workpiece disengagement (cut out) as the workpiece material is suddenly unavailable to dissipate heat” [24]. This finding is of relevance to the $\mathrm{AE}$ entry and exit spike phenomenon, as it has been already been shown by Webster [2] that acoustic amplitudes are increased as a result of thermal damage.

Perhaps the most interesting remark made by Malkin [24] in the previous quotation is that workpiece temperatures rise when the surrounding material becomes instantly unavailable to conduct away excess heat. This raises the new concept of variable engagement/disengagement geometry which would similarly affect workpiece temperatures in this area. Snoeys et al. [30] summarized several studies regarding thermal damage in grinding, noting that in the case of a wedge-shaped workpiece edge, the maximum temperature can be modeled as a function of the wedge-angle. It was also found that "very high temperatures are to be expected" at the edges, mainly due to less bulk material acting as a heat sink. These ideas will be used as a basis when investigating AE spike behavior in Chapter 4.

Many of the current thermal simulations can become complex, especially with the growing need for increased model performance. This introduces the new challenge of accurately selecting the physical system parameters as opposed to using previously simplified values. The term 'wheel-workpiece contact length' has occurred several times thus far, but its importance has not yet been adequately stated. Malkin et al. state that the "heat input to the workpiece occurs over the wheel-workpiece arc length of contact which can be approximated by the chord length” [24], while Mao et al. report that "the geometric contact length is used to approximate the real heat source length for simplifying the model” [31]. 
With one of the most significant parameters over the outcome of the theoretical grinding temperature model being the size and shape of the heat source zone [31], the actual contact length becomes paramount. The following section will hence introduce the role of the contact length in grinding and discuss current methods of its evaluation.

\subsection{Contact Length}

The contact length between the wheel and the workpiece is a fundamental parameter in grinding, and it has been an area of research for decades [32]. As previously mentioned, there is a common misconception regarding the actual size of the contact length, with many approximations based on the variations of the geometrically calculated value. This simplification can "cause an unexpected deviation" [32] from reality, as well as result in overpredicting grinding temperatures if a misleadingly small contact length is input into common thermal models [33].

Rowe reports that the "total contact length is typically 1.5 to 3 times the geometric contact length”, but this value has been seen to reach even higher values depending on grinding conditions $[33,34,35]$. The main reason for this is the elastic deformation within the grinding zone due to the applied normal force at the interface. Figure 2.12 illustrates Rowe's contact length model, which is dependent on three individual components; "[i] the geometric grinding contact zone, [ii] the elastic deflection between the wheel body and the workpiece and [iii] the microscopic contact at the grain level” [34]. 


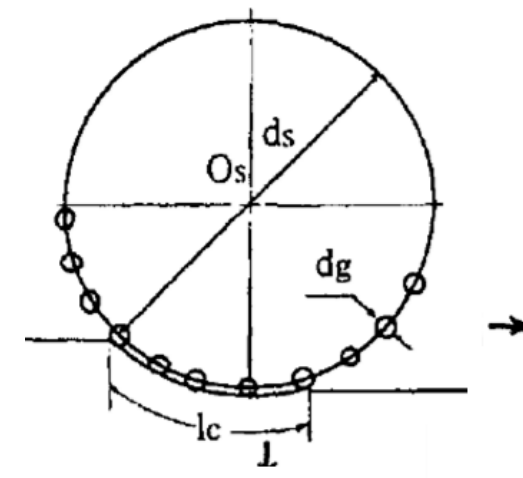

(a) The grinding wheel and workpiece contact during grinding

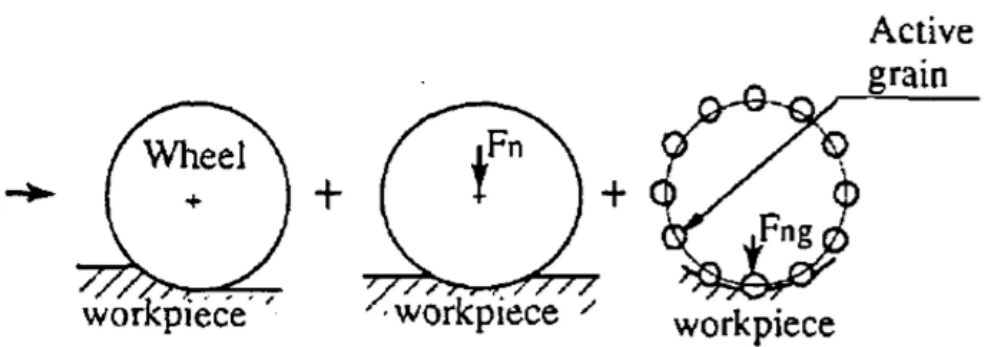

(b) The geometric contact (c) The elastic contact (d) The microscopic contact level

Figure 2.12: Rowe's model of the grinding wheel and workpiece contact [34].

The radius of the grinding wheel and the workpiece contact length each increase as the normal force is exerted during grinding. This elastic deformation results in the actual contact length to become greater than the geometrically calculated value, introducing the need for accurate measurement techniques. There have been several different developments over the past two decades for measuring the actual contact length during surface grinding. These techniques can be categorized as either a static or dynamic method, depending on if the calculation is performed post-process or during actual machining.

Static techniques involve gaining "information about the contact length and the profile of the contact zone by obtaining traces of the grains left on the surface of the workpiece through suddenly interrupting the grinding process” [32]. These methods include utilizing quick-stop devices, similarly employed for chip formation analysis during cutting, or Wager's “Two-Half Slot” grinding technique illustrated in Figure 2.13. 

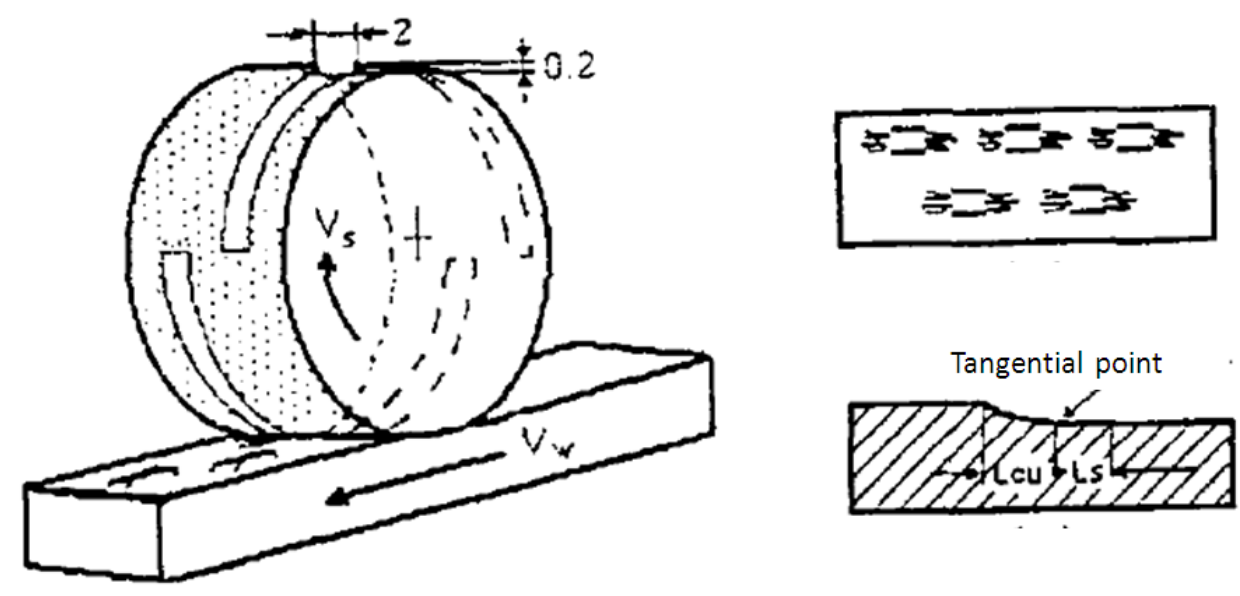

Figure 2.13: Two-Half Slot grinding technique [36].

This method is based on conventionally grinding a workpiece sample, but using a strategically slotted wheel. The consequence of the slotted regions result in sections of the workpiece remaining unground which can be analyzed post-process. The geometry and contour of the raised workpiece edges can be used to gather an understanding of the contact zone and approximate the actual contact length. However, this method can become extremely tedious and expensive if multiple grinding wheels are to be investigated under a wide variety of machining parameters.

Dynamic measurement techniques are more practical in the sense that they do not require an additional step of workpiece analysis following the grinding pass. The 'Peklenik technique' [32] appears to be the first to measure the contact length in-process, primarily based on thermocouple response, and is illustrated in Figure 2.14. 

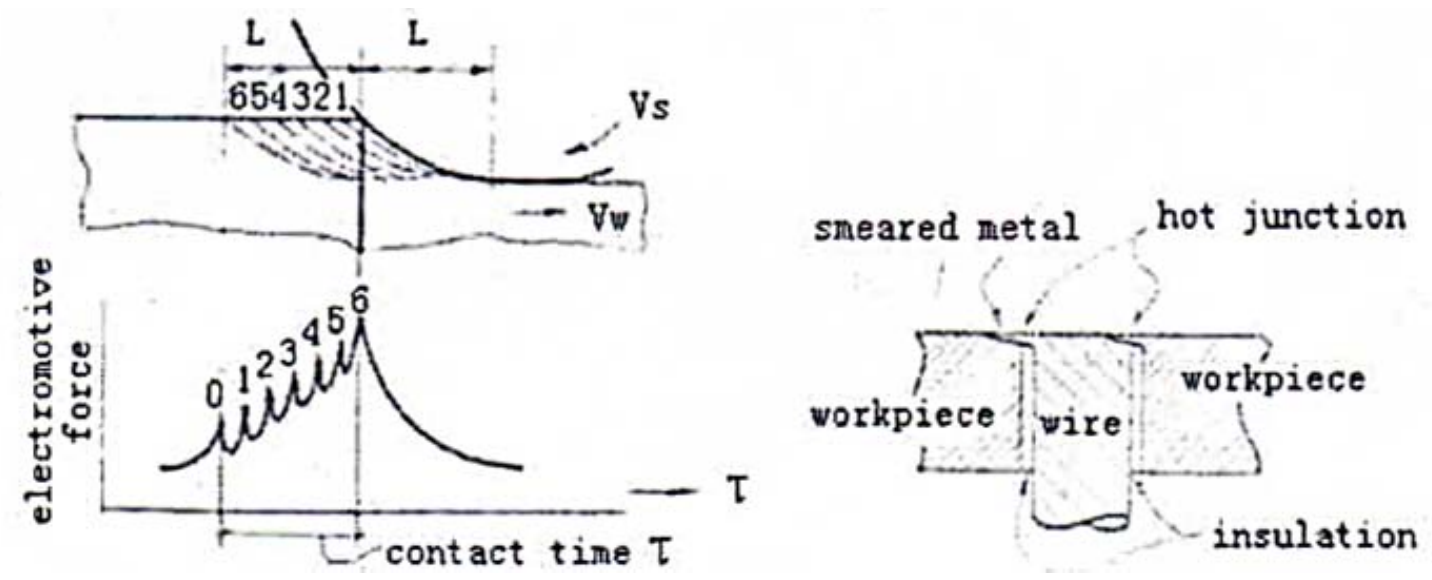

Figure 2.14: Peklenik method for measuring contact length [32].

This method relies on a specially prepared workpiece which has a small insulated wire embedded into it, forming a thermocouple. When "the workpiece is ground, a small amount of platinum from the tip of the wire is smeared over the workpiece surface by the rubbing of cutting action of the grinding grains” [32]. The contact length can be determined using the workpiece feedrate and contact time which corresponds to the distance that the hot junction travels relative to the grinding wheel surface.

However, this technique can occasionally become unstable if the thermocouple extends too deeply under the workpiece surface, causing a partial short-circuit of the temperature response [32]. This issue was later resolved by increasing the thickness of the insulation material shown in Figure 2.14, introducing the new widely accepted Critical Contact State mode of measuring the contact length.

Although other dynamic measurement methods exist, such as the “Applied Power Source (APS) method” $[32,35]$, they are all based on the original Peklenik technique. This approach is the most recent adaptation of the thermocouple method, but employs a single chromel foil pole acting as the power source contact probe. Figure 2.15 shows the experimental setup 
configuration for the APS and traditional thermocouple measurement procedure which is currently "the most reliable technique for sensing contact between the grinding wheel and the workpiece” [35].
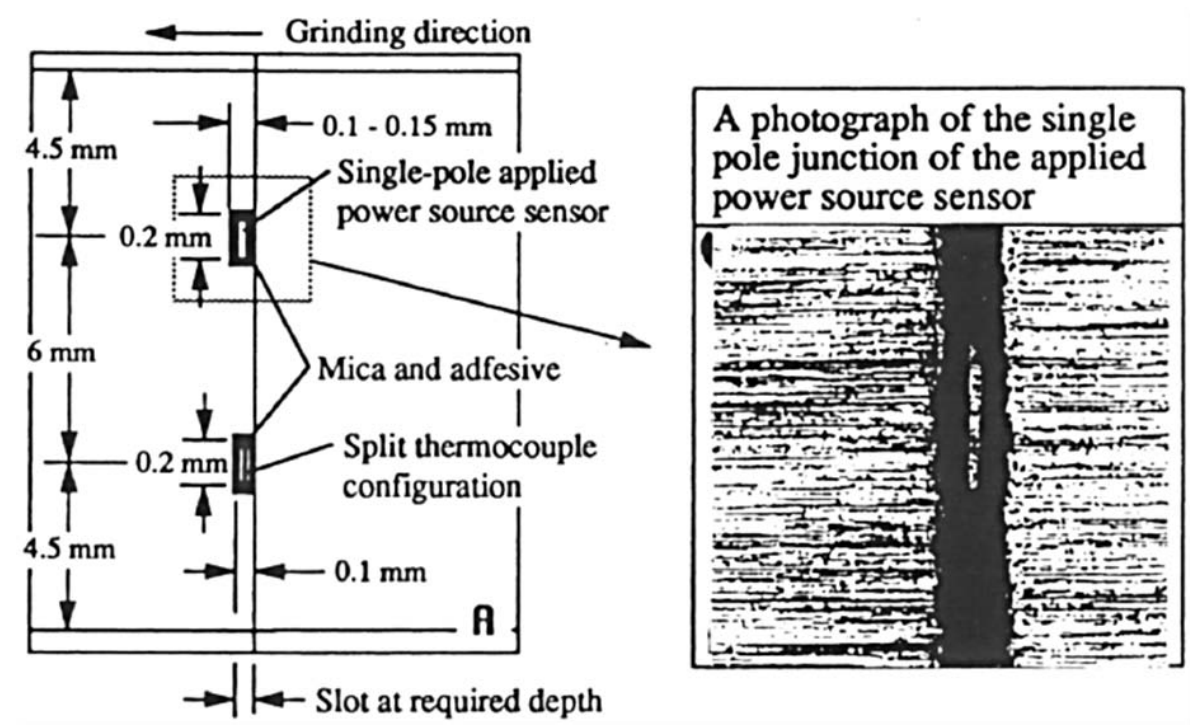

Figure 2.15: Setup configuration of the thermocouple junctions [35].

The unique workpiece setup for contact measurement can be seen to be quite intricate. Testing must be performed on these specifically designed samples and not the actual part being ground, which is the biggest drawback to all currently available contact length measurement techniques. Even though this dynamic method is more reliable than static methods, it still involves a generally difficult and complex procedure that must be re-examined for every change in grinding conditions.

However, these methods are the only way to physically verify models of contact length. The experimental results are expected to be greater than the geometric contact length as previously discussed, and are shown in Figures 2.16, 2.17, and 2.18, comparing individual works on the subject. 


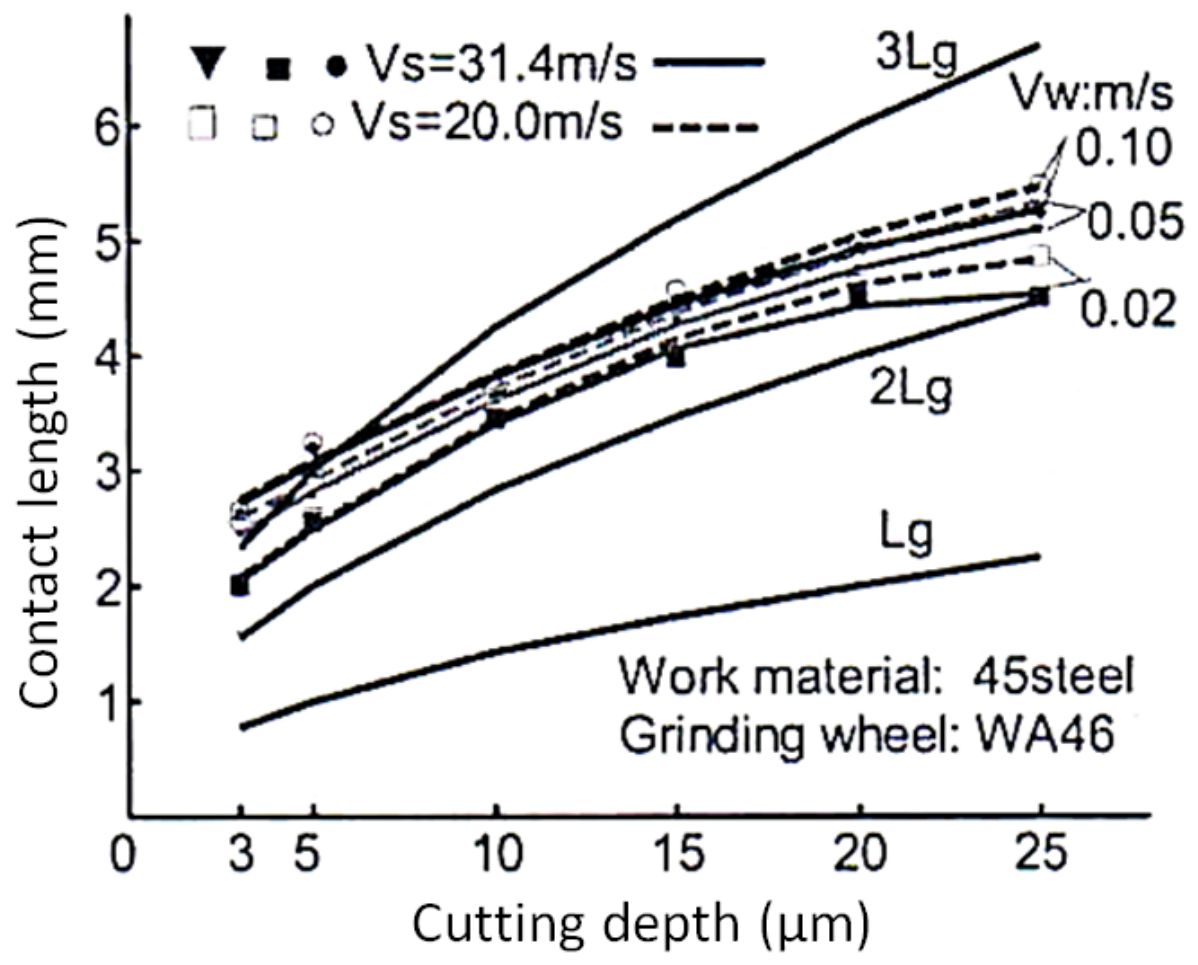

Figure 2.16: Contact length comparison using the Critical Contact State mode by Mao et al. [32]. Lg refers to the geometric contact length, calculated using ideal wheel-workpiece contact geometry.

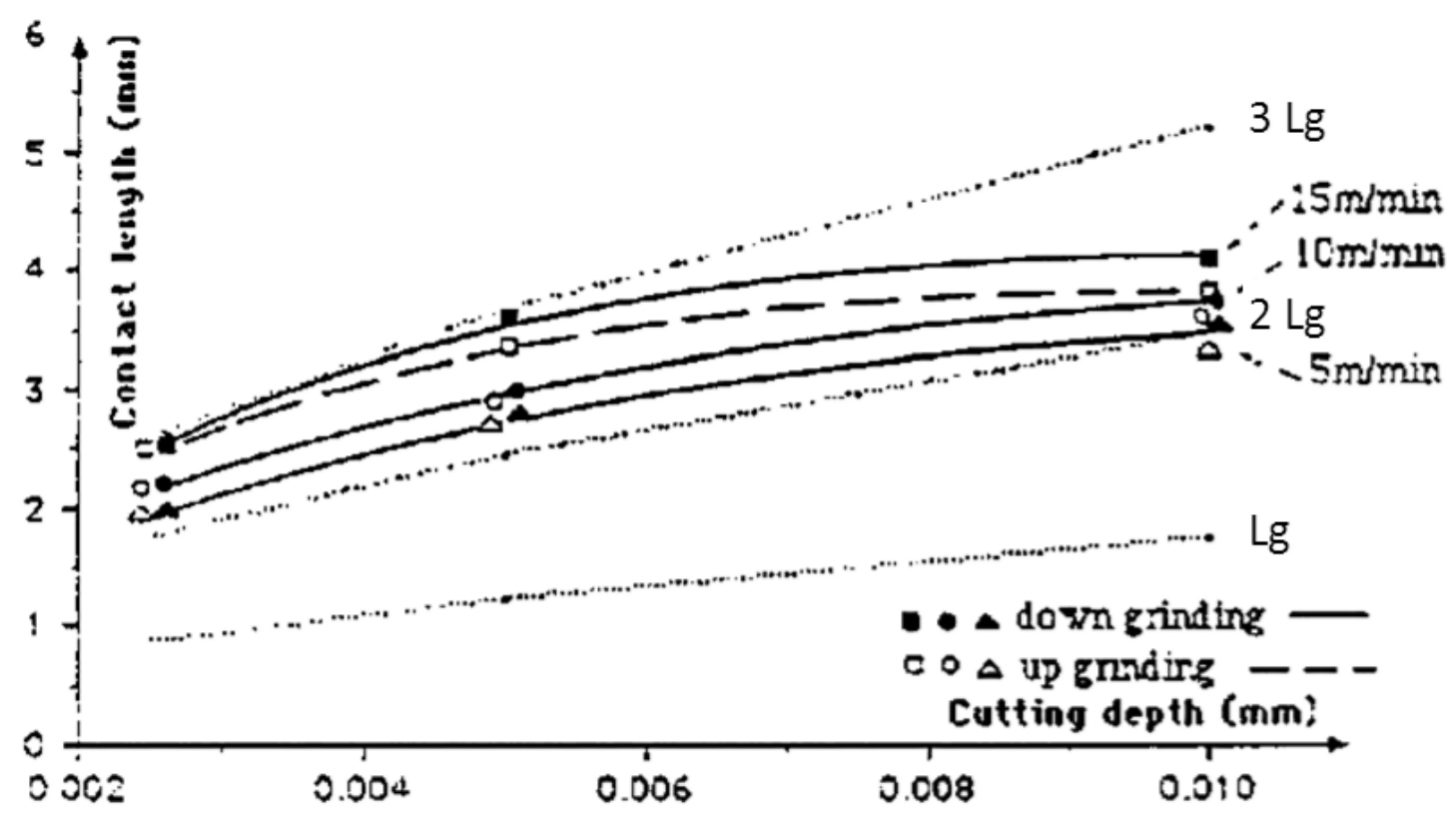

Figure 2.17: Contact length comparison using the Critical Contact State mode by Wager et al. [36] 


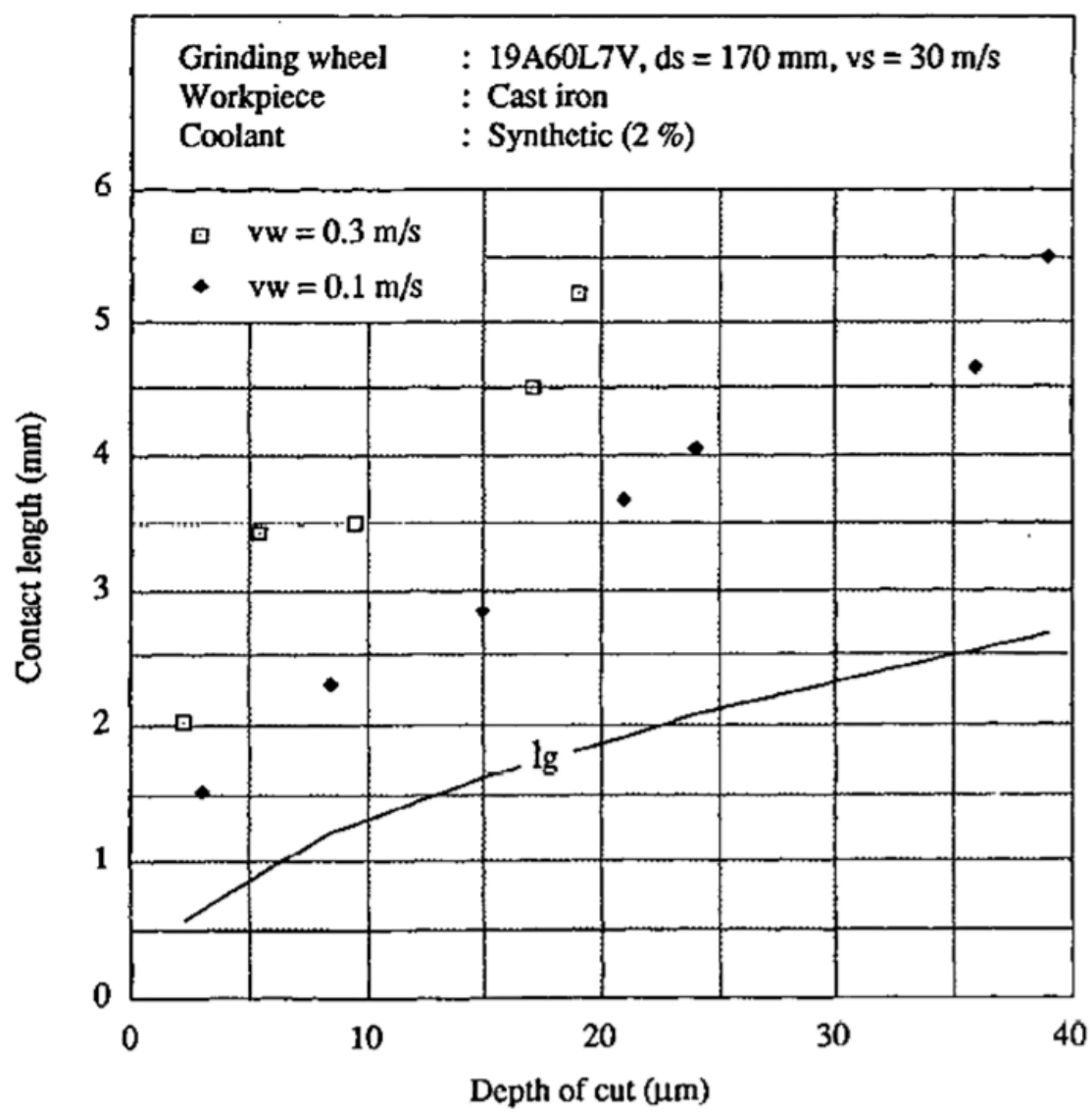

Figure 2.18: Contact length results using the Applied Power Source method by Qi et al. [35]

These independent results all show a similar trend concerning the measured contact length during surface grinding. The contact length can be seen to be "much larger than the value predicted from geometry" [35] alone, typically ranging between two and three times the geometric contact length. The results also show that the contact length increases at higher table feedrates, agreeing with theory referring to the relationship between normal force and elastic deformation.

Additional comprehensive reports of the contact length under varying grinding conditions can be found in the literature $[32,34,35,36]$, but they all generally conclude the same. The most important finding among all works is that the difference between the actual and geometric 
contact length can range between $101 \%$ and $600 \%$ [37] depending on the grinding application, with most ratios falling between $150 \%$ and $300 \%$. This large variance becomes problematic when accurately attempting to predict grinding temperatures, making the development of a new practical technique to measure the correct contact length value of much significance.

The contact length is not only useful for thermal modeling, but is also essential in understanding grinding system dynamics; including contact stiffness, chatter, and damping, as detailed in the following.

\subsection{Chatter, Damping and Grinding Dynamics}

In dealing with the surface grinding process, it becomes paramount that machining accuracy and productivity is controlled and optimized during production. Yamada et al. [38] state that "one of the problems in grinding operation is the elastic deformation of the grinding wheel by the influence of contact stiffness." This term refers to the relationship between the normal compressive force and the elastic deformation of the grinding wheel in the contact zone [39]. Inasaki reports that in general, "the grinding system becomes less stable as grinding stiffness increases" and that increases of the depth of cut, speed ratio $\left(\mathrm{v}_{\mathrm{w}} / \mathrm{v}_{\mathrm{s}}\right)$, and wheel hardness all lead to increases in contact stiffness [39].

However, in mass, spring, damper models of the grinding system, more than just the contact stiffness becomes important. Grinding damping is generalized as the "coefficient between the grinding force and the mutual approaching speed" of the grinding wheel and the workpiece [39]. Proportional increases in damping are due to relative increases of the contact length in the contact zone, which "has the effect of making the system more stable" [39]. 
Self-excited grinding chatter is directly associated with the stability of the process, being influenced by both the contact stiffness and damping parameters. This undesirable phenomenon has been a popular area of machining research, with numerous developments on its detection and control. Although it has been found that "normal force is more sensitive to chatter vibration than AE” techniques [40], it has also been reported that tool chatter indirectly influences the $\mathrm{AE}$ sources, causing some changes to the signal [7].

This is of particular interest when the AE spike phenomenon is considered, as many of the features associated with grinding can be related, as shown in Figure 2.19.

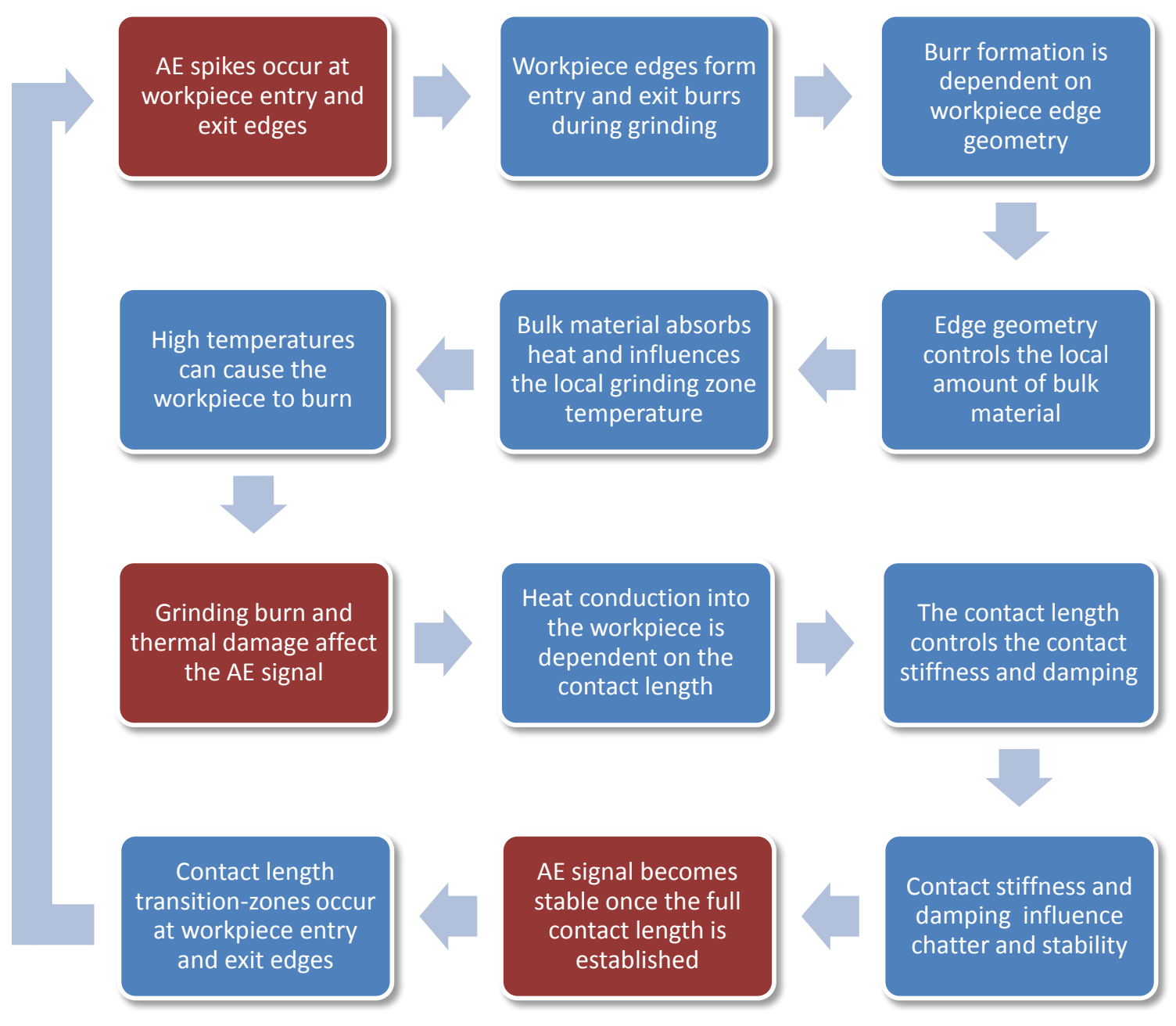

Figure 2.19: Relationship between various grinding features and parameters in terms of the AE spike phenomenon. 
A review of the literature relating to the acoustic emission phenomenon and surface grinding has been presented in this chapter. The following chapters will next outline the experimental methods used in this work, discuss the corresponding results, draw conclusions and outline avenues for future work. 


\section{Experimental Details}

The focus of this thesis is on interpreting the AE RMS spike phenomenon that occurs at workpiece entry and exit during surface grinding. As the project is curiosity-based, experiments were designed and performed to gain insight into AE spike formation. Specific details of the experimental setup, including equipment and its implementation, as well as the various experimental and data processing techniques used in the work are presented in this chapter.

\subsection{Machine Tool Setup}

Surface grinding experiments were performed using the Blohm Planomat 408 in the Machining Systems Laboratory of McMaster University. The machine tool, shown in Figure 3.1, is fully programmable and computer numerically controlled, capable of executing intricate and accurate movements.

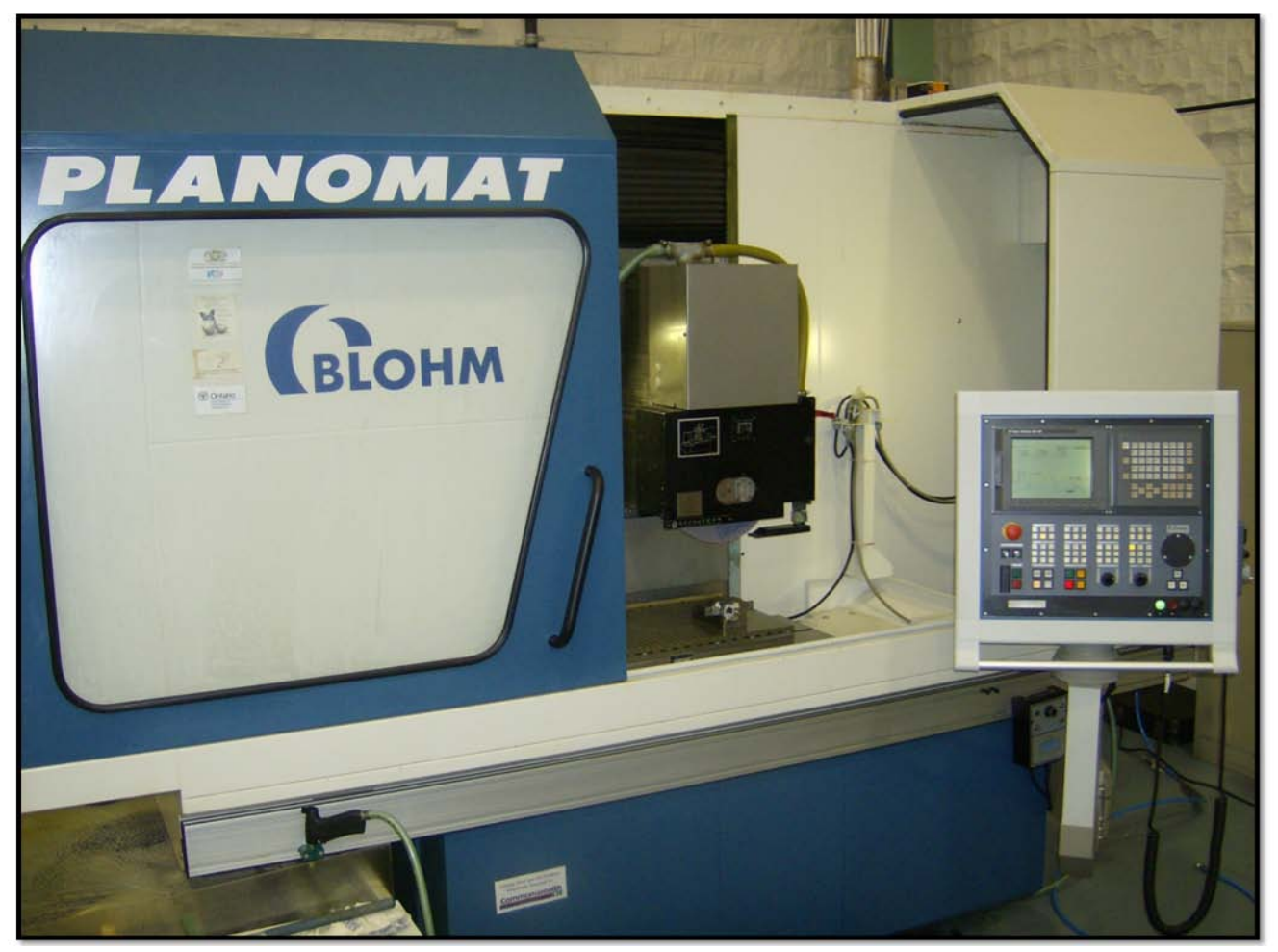

Figure 3.1: BLOHM Planomat 408 surface grinder in the MSL of McMaster University. 
Simple G-codes were input into the machine tool console to define grinding passes across the surface of a workpiece. Figure 3.2 illustrates the general path of the grinding wheel with respect to the workpiece.

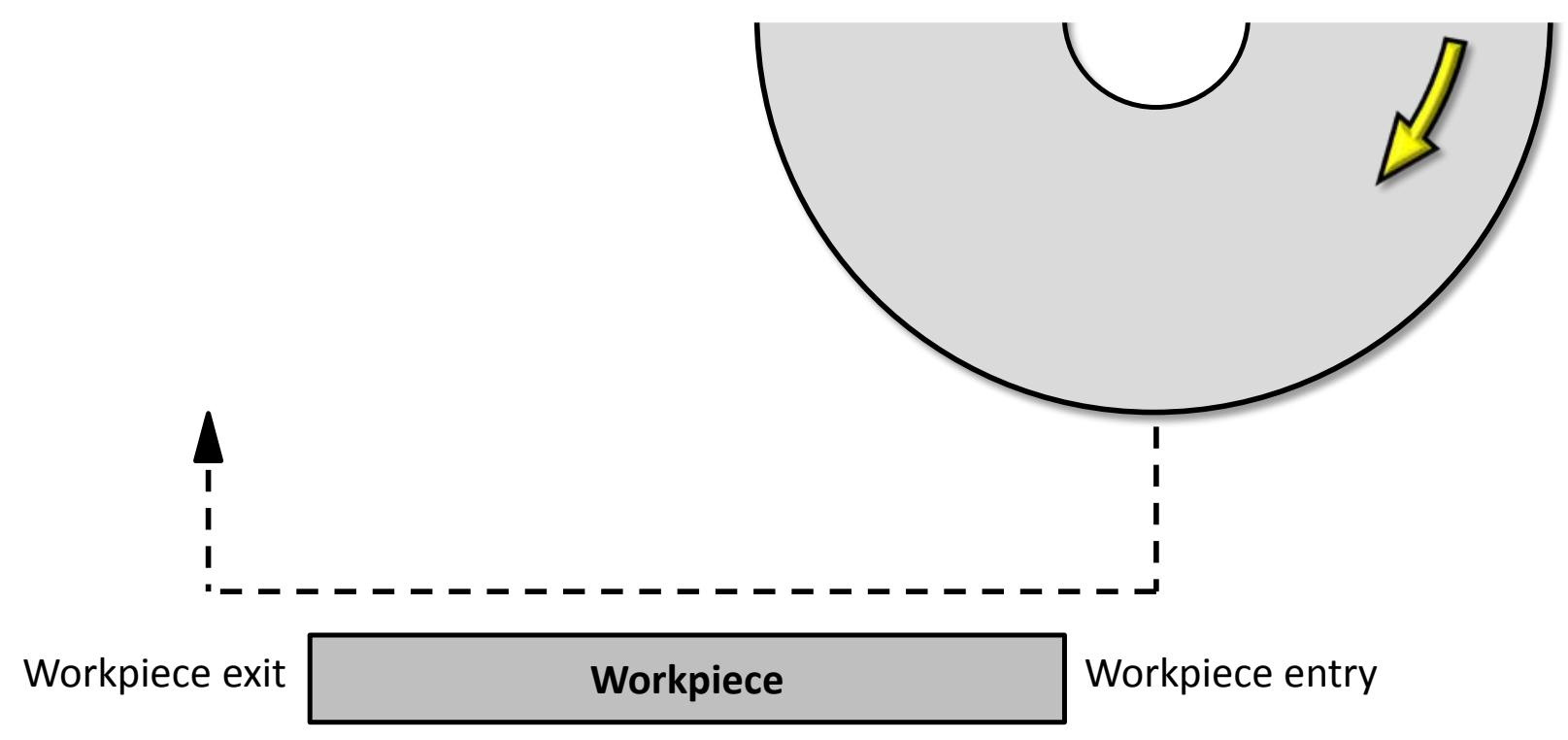

Figure 3.2: Relative tool path of an individual grinding pass. This direction represents up-grinding.

Note that the wheel does not plunge directly down into the workpiece upon approach, but enters and exits outside of the confines of the workpiece. It should also be clarified that this represents the relative tool path of the grinding wheel, as it is the workpiece being fed laterally during the pass.

Many parameters are included within the program, but the most significant ones are set to define spindle speed, table feedrate, depth of cut, and whether the grinding pass will execute as either up-grinding or down-grinding. Table 3.1 lists the baseline grinding conditions that were utilized for most experiments. 
Table 3.1: Baseline grinding parameters

\begin{tabular}{|l|l|}
\hline \multicolumn{1}{|c|}{ Parameter } & \multicolumn{1}{c|}{ Value } \\
\hline Grinding Wheel Surface Speed & $20 \mathrm{~m} / \mathrm{s}$ \\
\hline Table Feedrate & $10.2 \mathrm{~m} / \mathrm{min}$ \\
\hline Depth of Cut & $10 \mu \mathrm{m}$ \\
\hline Grinding Pass Direction & Up-Grinding \\
\hline Coolant & ON \\
\hline Workpiece Entry/Exit Geometry & $90^{\circ}$ \\
\hline
\end{tabular}

A straight-line wheel dressing program was also defined for the experiments, utilizing a single-point diamond dressing tool. Upon execution, a layer of the grinding wheel would be removed at specified wheel and table speeds, exposing new grinding grains. The machine is also programmed to recognize the decreasing wheel diameter after each dressing pass. This not only compensates for the work offset positions, but also regulates the rotational spindle speed in order to maintain a constant grinding surface speed. Table 3.2 lists the baseline dressing conditions that were used.

Table 3.2: Dressing parameters

\begin{tabular}{|l|l|}
\hline \multicolumn{1}{|c|}{ Parameter } & \multicolumn{1}{c|}{ Value } \\
\hline Grinding Wheel Surface Speed & $20 \mathrm{~m} / \mathrm{s}$ \\
\hline Cross-feed rate & $7.87 \mathrm{~cm} / \mathrm{min}$ \\
\hline Dressing Depth per Pass & $\sim 18 \mu \mathrm{m}$ \\
\hline Number of Dressing Passes & 7 \\
\hline Total Dressing Depth & $127 \mu \mathrm{m}$ \\
\hline
\end{tabular}


Referring to Table 3.2, the total dressing depth of $127 \mu \mathrm{m}$ is a radial value. This corresponds to the actual diameter of the wheel being reduced by approximately $254 \mu \mathrm{m}$. It is important that the dressing spindle speed is set to the same value that is desired during actual machining to avoid dimensional inaccuracy, as the diameter of the grinding wheel can vary with rotational speed due to the induced centrifugal force.

Grinding wheels come in a variety of shapes and types, but the most common straight edge wheel was used. The grinding wheel that was used for the majority of the testing phase is specified as grade 32-A-46-G-V-40P and supplied by Carborundum Abrasives. The grit size of the aluminum oxide wheel being used is fairly coarse with a very open grain structure. The increased porosity and tougher grains make this wheel suited towards grinding tool steels and other very hard materials. The size of the new wheel was 14 " $\times 1.5$ " $\times 5$ ", where the values indicate the dimensions of the outer diameter, wheel thickness, and inner bore diameter respectively.

It is very important to balance the grinding wheel before it is used to avoid eccentricity and run-out errors. The MPM BMT100 electronic grinding wheel balancer was used to identify the circumferential spindle positions at which balancing weights were to be attached. Aside from the grinding wheel setup, the coolant tank was also monitored to ensure proper concentration and fluid level. A handheld refractometer was used to check the concentration of the water-based full synthetic coolant, with an optimal value reading between $8 \%$ and $12 \%$. 


\subsection{Workpiece Setup}

Surface grinding tests were largely performed on hardened high speed steel (HSS) workpiece samples. This material was chosen for several reasons; its properties are similar to the high hardness alloys (50-55 HRC) that are commonly ground in industry; past research $[2,10,11]$ studies involving acoustic emission typically use HSS and other equally hard workpiece samples such as 52100 bearing steel; acoustic emission entry and exit spikes have been reported to exist during grinding of this type of steel.

HSS blanks of different sizes were used, but most workpieces came with the initial dimensions of approximately $12 \mathrm{~mm} \times 12 \mathrm{~mm} \times 100 \mathrm{~mm}$ in length. The surfaces were first preground and the two ends were ground to precise angles ranging from $5^{\circ}$ up to $135^{\circ}$ as seen in Figure 3.3. However, baseline experiments used $90^{\circ}$ entry and exit workpiece angles as noted in Table 3.1.
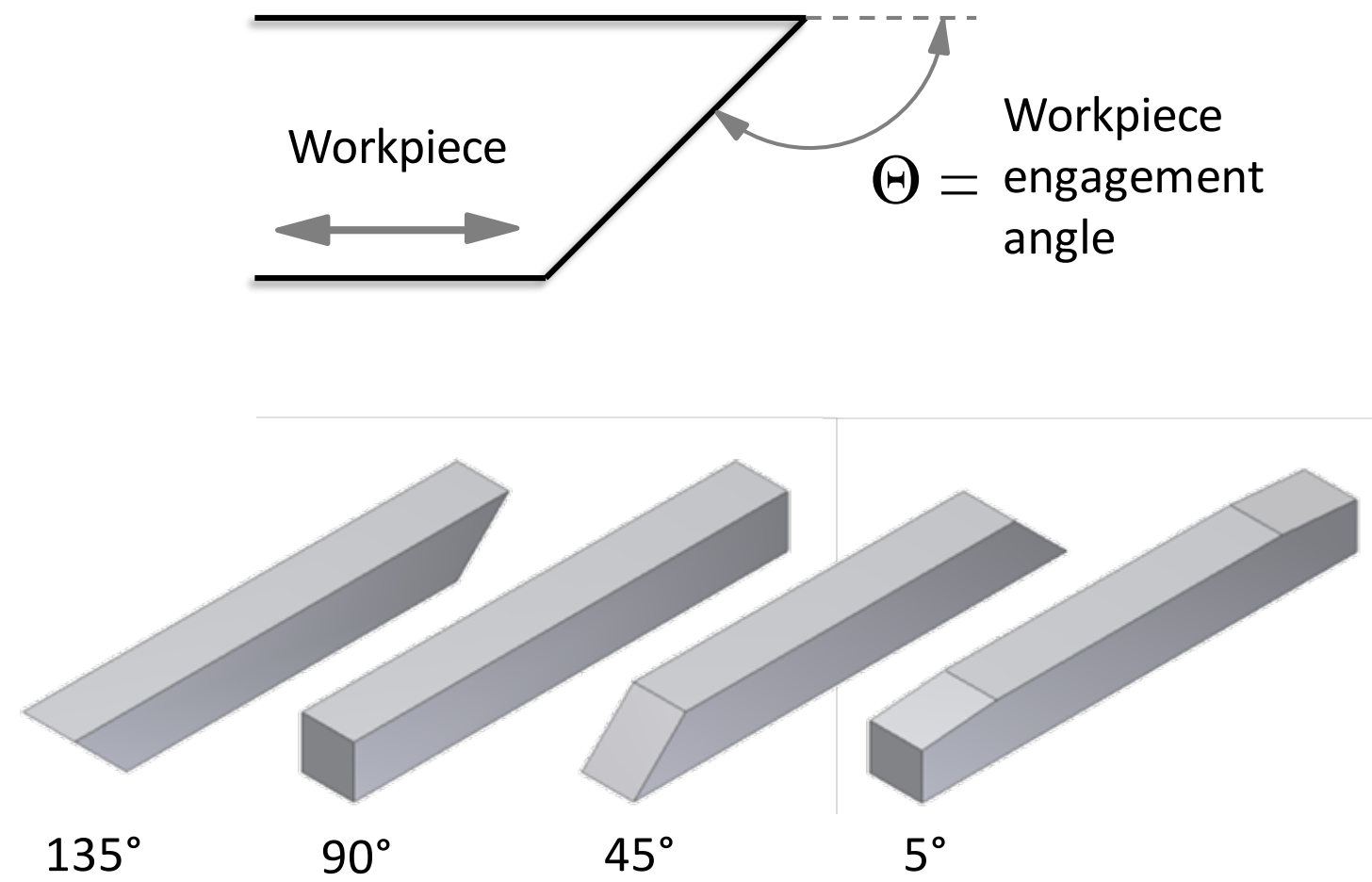

Figure 3.3: Workpiece engagement angles that define workpiece entry/exit geometry. 
The completed workpieces to be tested were held in a 3” grinding vice and magnetically mounted to the grinding table. Precision parallels were used underneath the workpiece in order to expose only a few millimeters above the top surface of the vice. The length of the workpiece was positioned parallel to the table feed direction so that a single grinding pass would completely cover the complete surface.

In order to obtain usable AE feedback of the surface grinding process, the proper use of both the sensors and data acquisition equipment must be assured. The following section will detail the procedure regarding this setup.

\subsection{Equipment Setup}

Care must be taken when configuring the acoustic emission data acquisition system. Keeping in mind that raw acoustic emission signal analysis requires sampling rates that can exceed $1 \mathrm{MHz}$, a powerful workstation must first be assembled with appropriate data acquisition hardware. A custom PC was built, supporting a 2 channel AE data acquisition and digital processing card from the Physical Acoustics Corporation (PAC). The 18-bit PCI-2 card is designed for AE analysis, capable of continuous waveform streaming to hard disk at up to 10 million samples per second. However, at sampling rates this high, hard drive write speed becomes one of the limiting factors to reliable data collection. Using a redundant array of independent hard disks (RAID) is a common method to account for increased write-speeds, but can include problems of synchronizing the time sensitive data afterwards. Therefore, a single solid state hard drive, capable of reading up to $250 \mathrm{MB} / \mathrm{s}$, was built into the PC, which replaces the need for a RAID setup. This additional hard drive is more than sufficient for the temporary 
storage of raw AE data. The PC was also equipped with the PAC AEwin software suite, as well as LabVIEW and MATLAB for data processing.

Kistler type 8152B111 acoustic emission sensors were used during the surface grinding experiments. The frequency response of the sensors is shown in Figure 3.4, which indicates an optimal sensitivity in the range of 50 - $400 \mathrm{kHz}$.

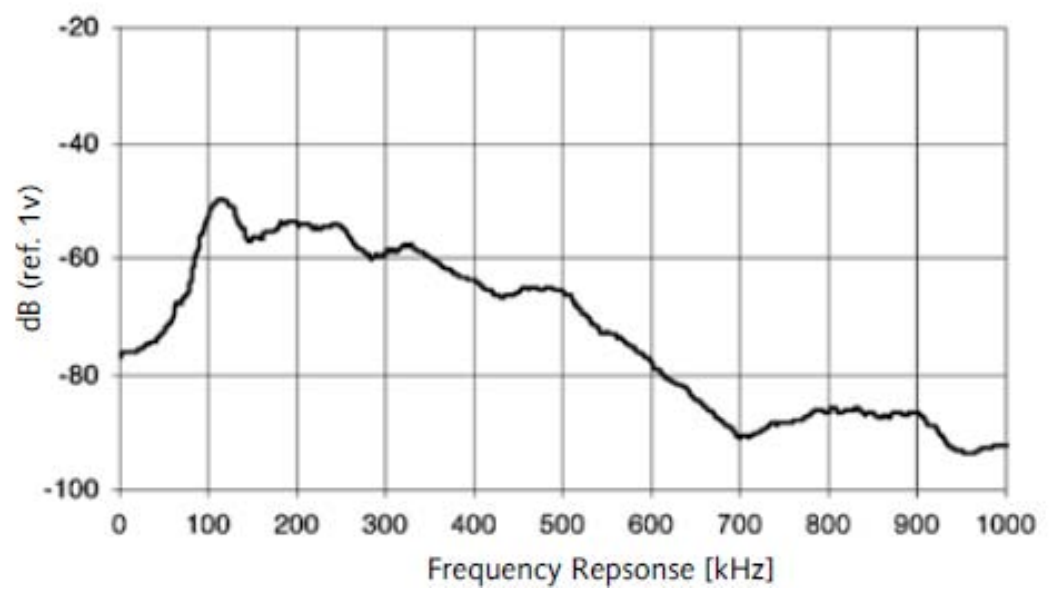

Figure 3.4: Frequency response of the AE sensor provided by the manufacturer [41].

The sensor setup must also include the 5125B1 Piezotron Coupler, which houses modular analogue high and low-pass filters, as well as an RMS converter with corresponding timeconstant. A $50 \mathrm{kHz}$ high-pass filter and $1000 \mathrm{kHz}$ low-pass filter were used, as well as a $1.2 \mathrm{~ms}$ RMS time-constant chip. This coupler also houses a physical gain jumper. It is vitally important that this gain be set to the $\times \mathbf{1}$ position to avoid over-saturating the AE signal during machining.

The AE coupler requires a $24 \mathrm{~V}$ power supply, and provides two useful output signals. The first is the Raw AE data which connects directly to the PCI-2 DAQ card, sampled at $2 \mathrm{MHz}$ per channel. The second is the calculated RMS data, connected to a more common USB data acquisition device. The National Instruments USB-9162 was used, with a sampling frequency of $20 \mathrm{kHz}$ for acquiring the RMS signal. 
The AE sensors were magnetically mounted to the vice as well as the grinding table in a position that minimizes acoustic interference. For the most effective AE monitoring, the sensor should be positioned as close to the grinding zone as possible. However, due to the high acoustic levels during machining, care must be taken not to position the sensors too close either, or portions of the signal may saturate the sensor output. Also, as the grinding wheel moves across the surface of the workpiece, the position of the acoustic event changes with respect to the stationary sensor. This can result in the signal amplitude increasing or decreasing as the grinding zone moves toward or away from the AE sensor. Therefore, by positioning the sensors along the midpoint of the workpiece length, as well as at an appropriate distance from the grinding zone, useful AE data can be collected. Figure 3.5 shows the general experimental setup used with physical locations of the sensors.

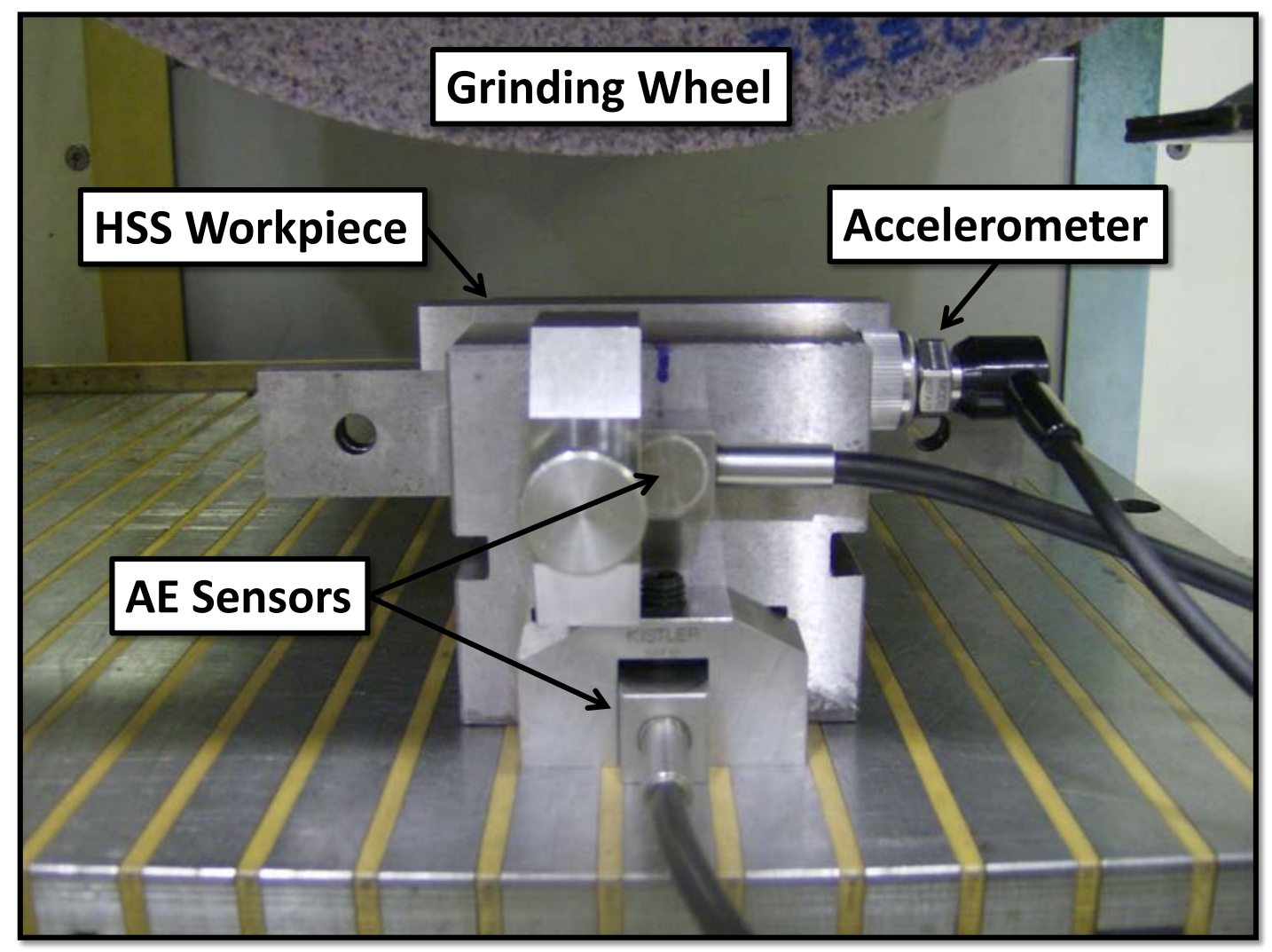

Figure 3.5: Experimental grinding setup with sensor positions. 
A common accelerometer was employed in addition to the AE sensors for comparative purposes. It was mounted to the vice parallel to the grinding direction at workpiece entry and exit locations.

\subsubsection{Note on AE Sensor Positioning}

Two AE sensors were implemented as seen in Figure 3.5. The first was positioned on the side of the grinding vice and the second was mounted directly to the table. The AE response of the primary sensor was found to be better suited for presenting data in this work as its location was closest to the grinding zone, providing a 'louder' and clearer representation of the wheel/workpiece interaction. The secondary table sensor was generally relied on for verification purposes.

In the case of some experiments, the primary AE sensor had to be positioned lower on the vice (further from the grinding zone) to prevent signal saturation. This becomes relevant for the data presented in Figure 4.10 for example, which was compared for varying depths of cut. More aggressive grinding parameters typically cause the AE signal amplitude to increase, and so the sensor had to be appropriately positioned to avoid saturating the sensor during several of these experiments. This explains why some of the AE amplitude axes differ between individual sets of results presented in Chapter 4.

As a focus of this work includes identifying relative $\mathrm{AE}$ trends under various grinding conditions, all comparisons are valid as long as the sensor position remains constant for each set of experiments. Therefore, all comparative sets of data presented in this thesis have been collected using a constant sensor location, granted that it may vary between specific cases to account for instances which cause the AE signal to otherwise exceed the range of the sensor. 


\subsubsection{AE Verification}

The validity of the collected experimental data is important to verify. In particular regards to acoustic emission, the hardware and software parameters must be appropriately set for the grinding experiments. As the main focus of this project is the interpretation of the RMS spikes which occur at workpiece entry and exit, it is crucial that the time-constant is such that the resulting RMS signal is a valid representation of the actual signal spikes that occur in the raw AE signal.

In previous research, Webster states that an integration time-constant of approximately $1.0 \mathrm{~ms}$ is sufficient for RMS signal analysis of the grinding process [10]. However, Jemielniak [13] later reported that if the RMS signal is to be used for AE burst analysis, the integration timeconstant should not exceed 0.1 ms. This is a significant discrepancy. The analogue RMS output from the Kistler equipment is calculated based on a $1.2 \mathrm{~ms}$ time-constant, so it must be determined if this signal is an accurate representation of the raw signal, or is distorted due to an excessive time-constant value as stated by Jemielniak. Figure 3.6 shows an example of Jemielniak's results. The raw AE data is displayed above the corresponding RMS signal outputted by the same Kistler equipment and time-constant $(1.2 \mathrm{~ms})$ used for current testing.

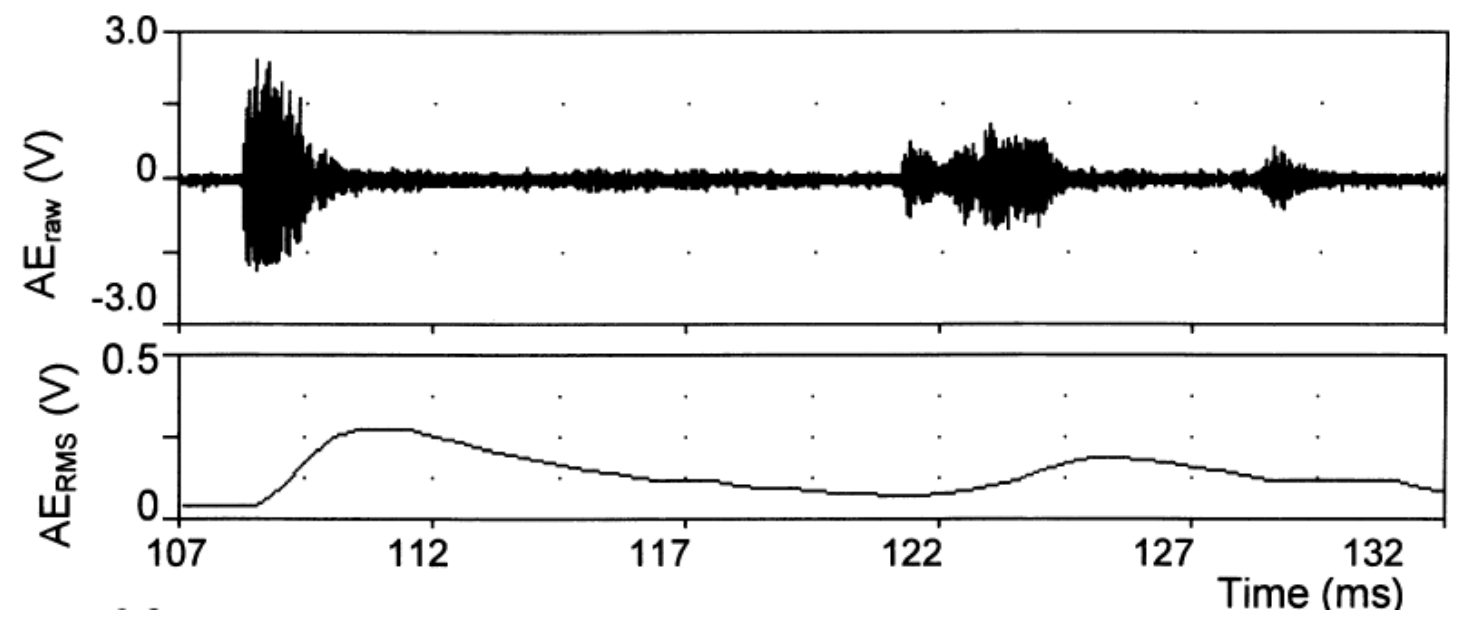

Figure 3.6: AE Raw and RMS signals of lead breaking using 1.2 ms time-constant [13]. 
It can be seen that the raw $\mathrm{AE}$ burst is not accurately represented by the RMS characteristic using this time-constant. However, the AE bursts reported here are approximately 2-3 ms in duration. When looking at the typical duration of workpiece entry and exit spikes being investigated during surface grinding, the time length is approximately 10 times longer; ranging between 20-30 ms. This proves several key points. The first is that AE entry/exit spikes seen during grinding are not typical AE burst-type signals. These spikes are not simply caused by a single event, but are sustained over a certain time length. Because the spike duration is approximately $10 \times$ longer than the 2-3 ms duration of single AE bursts, the corresponding timeconstant can be $10 \times$ larger than the Jemielniak's proposed $0.1 \mathrm{~ms}$. Figure 3.7 shows how the ratio between the raw spike duration and the RMS spike duration changes as the time-constant is varied.

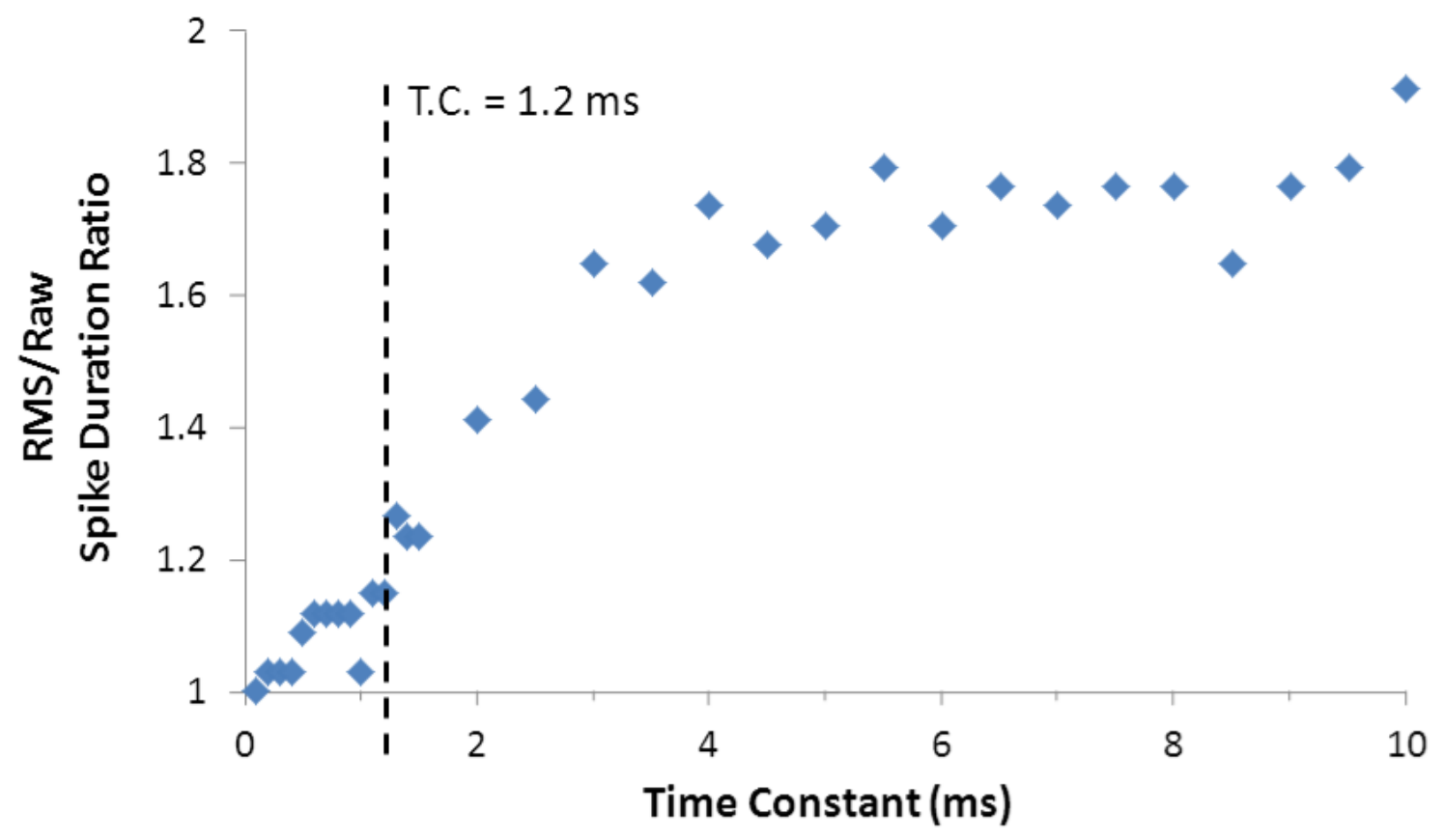

Figure 3.7: RMS/Raw spike duration ratio as a function of RMS time-constant. Significant divergence begins above $1.2 \mathrm{~ms}$. 
Using a time constant of 0.1 to $1.2 \mathrm{~ms}$ provides an RMS spike length that falls approximately within $15 \%$ of the raw spike length values. It can therefore be said that the $1.2 \mathrm{~ms}$ time constant will provide accurate results during surface grinding experiments. However, note how error accrues as the time constant is increased, agreeing with Jemielniak’s findings.

It may be argued that the AE spike is influenced by the settling time of the sensor. This can be proved to be of no consequence by simply examining a typical RMS response, presented in Figure 3.8.

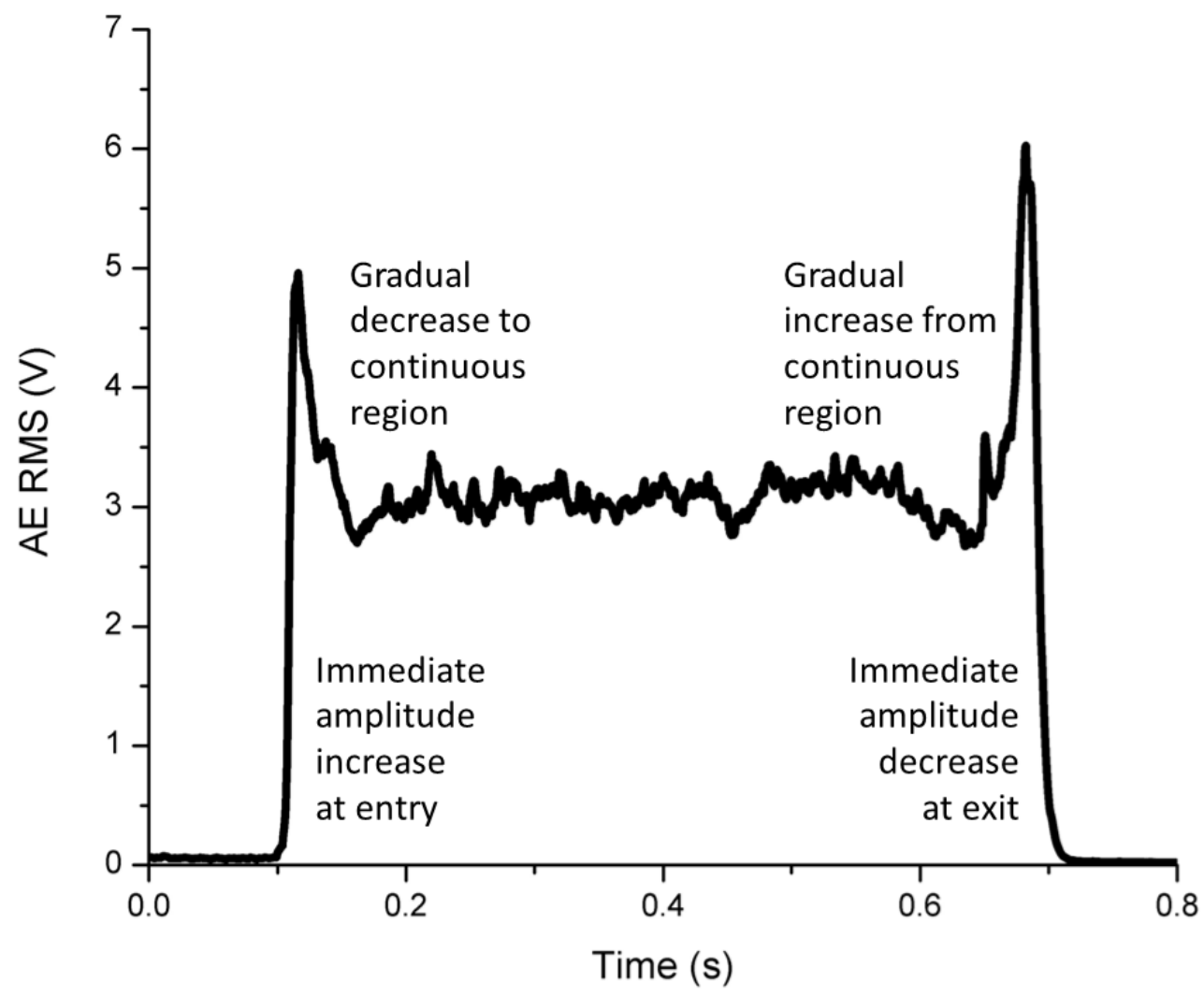

Figure 3.8: AE RMS entry and exit spikes are not significantly influenced by the settling time of the sensor.

It can be seen that the entry spike rises to its maximum almost immediately, followed by a more gradual decrease to the continuous region. If this was indeed a result of settling time, then the exit spike would behave in the same way. However, it can be seen that for this spike, its 
amplitude gradually increases to the maximum, and then almost immediately drops to zero, proving that signal settling time is of insignificant influence. In addition to this, the attenuation of a single AE burst is typically in the range of a millisecond or two. This means that AE bursts rapidly occur and then disappear almost immediately due to their transient nature. As previously stated, the typical entry and exit spike durations range upwards of $20 \mathrm{~ms}$, further proving that an attenuation time of only 1-2 ms can be considered insignificant.

Another aspect of AE signal processing to consider also refers back to Jemielniak's reports [13]. He states that the AE amplifier gain should be as small as possible to avoid overloading the pre-amplifier and distorting the signal. This is indeed true, as preliminary tests failed to yield any AE spikes due to incorrect gain settings, causing saturation of the entire signal.

Other researchers [42] have reported that AE spikes are not at all related to the grinding process, but are a result of increased signal gain during workpiece entry and exit. This is a prime example that shows how this phenomenon is indeed misunderstood, commonly being avoided or passed off on as a signal processing issue. The following chapter will outline the findings based on the workpiece entry and exit spikes, illustrating that they are not merely a random occurrence, but can be used to provide information that is of remarkable practical significance. 


\section{Results and Discussion}

As detailed in the previous chapters, acoustic emission spikes at workpiece entry and exit during surface grinding are a phenomenon that is yet to be explained or interpreted to yield useful information. Therefore, the scope of this project referred to understanding the spike occurrence, possibly leading into potential applications. The results obtained to this end are presented in this chapter, and will first pertain to RMS observations, with an investigation of the factors contributing to $\mathrm{AE}$ entry/exit spike behavior. A more in-depth analysis of the raw $\mathrm{AE}$ signal follows.

\subsection{AE RMS Signal}

This section focuses on the AE RMS signals observed during surface grinding experiments. Trends and observations relating to acoustic emission entry and exit spike behavior are presented to demonstrate the effect of various grinding conditions.

\subsubsection{Effect of Successive Grinding Passes and Burr Formation}

Grinding burr formation was noticed almost immediately following preliminary surface grinding experiments. Early hypotheses were made that linked the AE entry and exit spikes to grinding burr formation at the corresponding workpiece locations. Experiments were performed to study the effect of successive grinding passes on burr growth and the AE response, as well as the effect of pre-existing entry and exit burrs, for comparison with deburred workpiece edges.

Figure 4.1 shows the AE RMS signals corresponding to nine individual surface grinding passes using the baseline machining parameters listed in Table 3.1. A single HSS workpiece with $90^{\circ}$ entry and exit angles was used for this set of observations. 


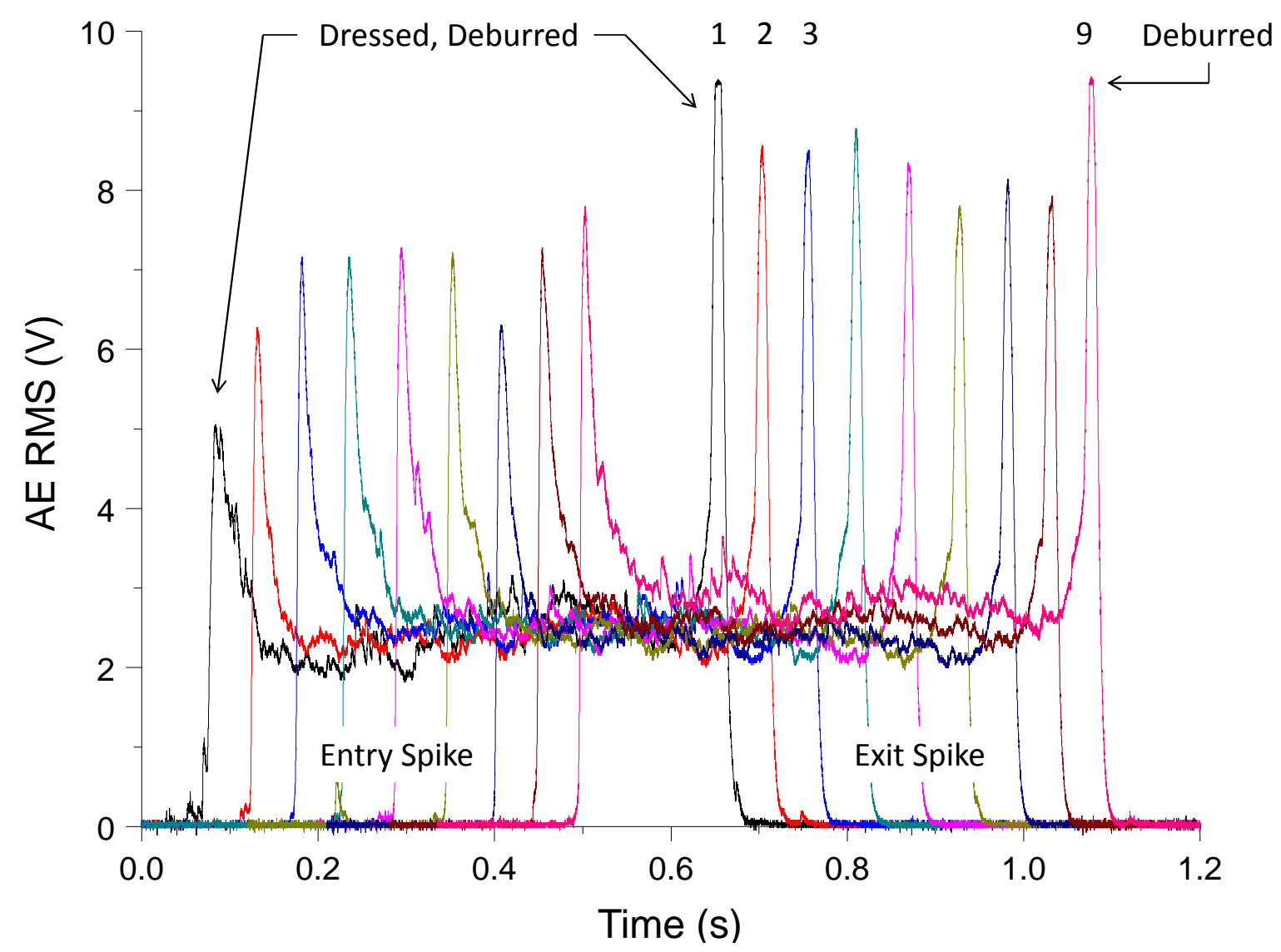

Figure 4.1: AE RMS results from successive up-grinding passes (1-9). Grinding wheel dressed prior to Pass 1, workpiece exit edge deburred before Pass 1 and Pass 9 only.

The first signal corresponds to a grinding pass using a freshly dressed wheel across a fully deburred workpiece. The following RMS signals (passes 2-8) relate to consecutive grinding passes without redressing the wheel or removing any workpiece burrs in between. The exit burr was removed just before the very last RMS signal was collected (pass 9) to elucidate the effect of burr formation.

It can be seen that burr formation has a noticeable effect on the amplitude of the RMS spikes. Exit burrs were found to be more significant than entry burrs during up-grinding, and so the corresponding AE exit spikes will be discussed first. 
The first grinding pass, shown in Figure 4.1, with an initially deburred workpiece exit edge yields the greatest AE exit spike. The amplitude of the exit spikes tends to become lower in the following grinding passes, until it instantly increases again once the exit edge is deburred (pass 9). This could be due to several reasons. The first refers to plastic deformation and material flow at the deburred workpiece exit edge. The majority of the burr growth occurs immediately following this grinding pass, as the workpiece edge goes from having no burr at all to one with noticeable overhang. Microscopic images of the exit burr are shown in Figure 4.2, taken after select passes from Figure 4.1.

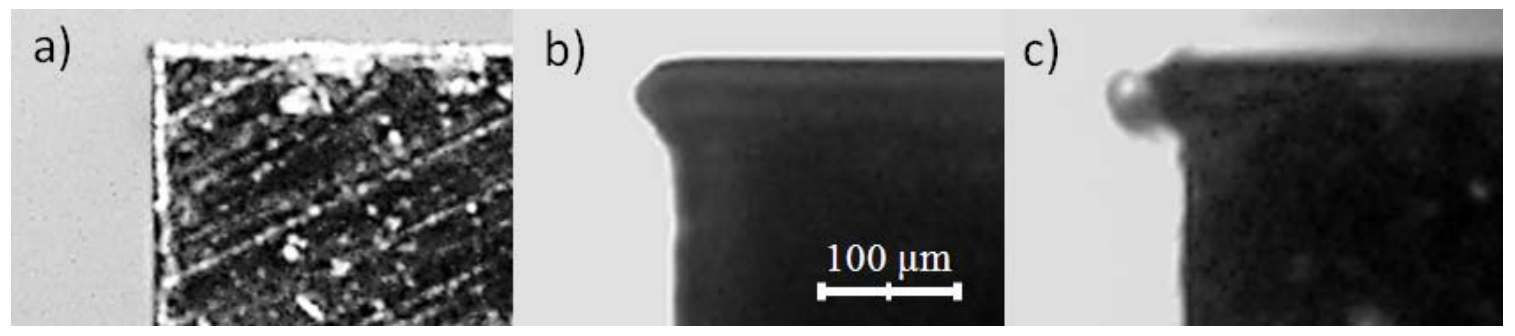

Figure 4.2: Microscopic images of workpiece exit burr. Image a) shows the deburred exit edge, b) and c) are taken of the same edge following grinding passes 1 and 5 of Figure 4.1, respectively.

A comparison of the deburred exit edge to that following grinding pass 1 and pass 5 is shown. Significant formation of the exit burr is noticed immediately after deburring. Successive passes only grow the burr slightly, but not with the same magnitude as the initial pass. As plastic deformation within a material is a source of acoustic emission, this indicates that it is a contributing factor in the increased $\mathrm{AE}$ exit spike following workpiece deburring. Another reason that could explain this occurrence is discussed in a later section as it refers to grinding burn. 
An interesting observation from Figure 4.1 can also be made regarding the entry spikes. It can be seen that the AE entry spike amplitude gradually increases over the first several passes. The initial up-grinding pass after deburring does not yield any significant entry burrs, however, they do become slightly evident after several successive grinding passes. As the entry burr gradually grows, the amplitude of the AE entry spike also increases. This is in contrast to the immediate jump in the AE exit spike amplitude following deburring, but is still based on the same principle.

Entry burr formation is much more gradual during up-grinding than exit burr formation on an initially deburred workpiece. This progressive rate of burr formation follows patterns in the AE RMS signals, with the difference between consecutive spike amplitudes seemingly related to relative burr growth. As these results are solely based on up-grinding experiments, the following section will hence introduce the effect of grinding direction on both burr formation and the AE spike phenomenon. 


\subsubsection{Effect of Grinding Direction}

In order to investigate the effect of grinding direction on the AE RMS entry and exit spikes, the baseline grinding parameters listed in Table 3.1 were kept constant, while changing the feed direction. Figure 4.3 shows the common AE RMS results of up-grinding compared to down-grinding following workpiece deburring.

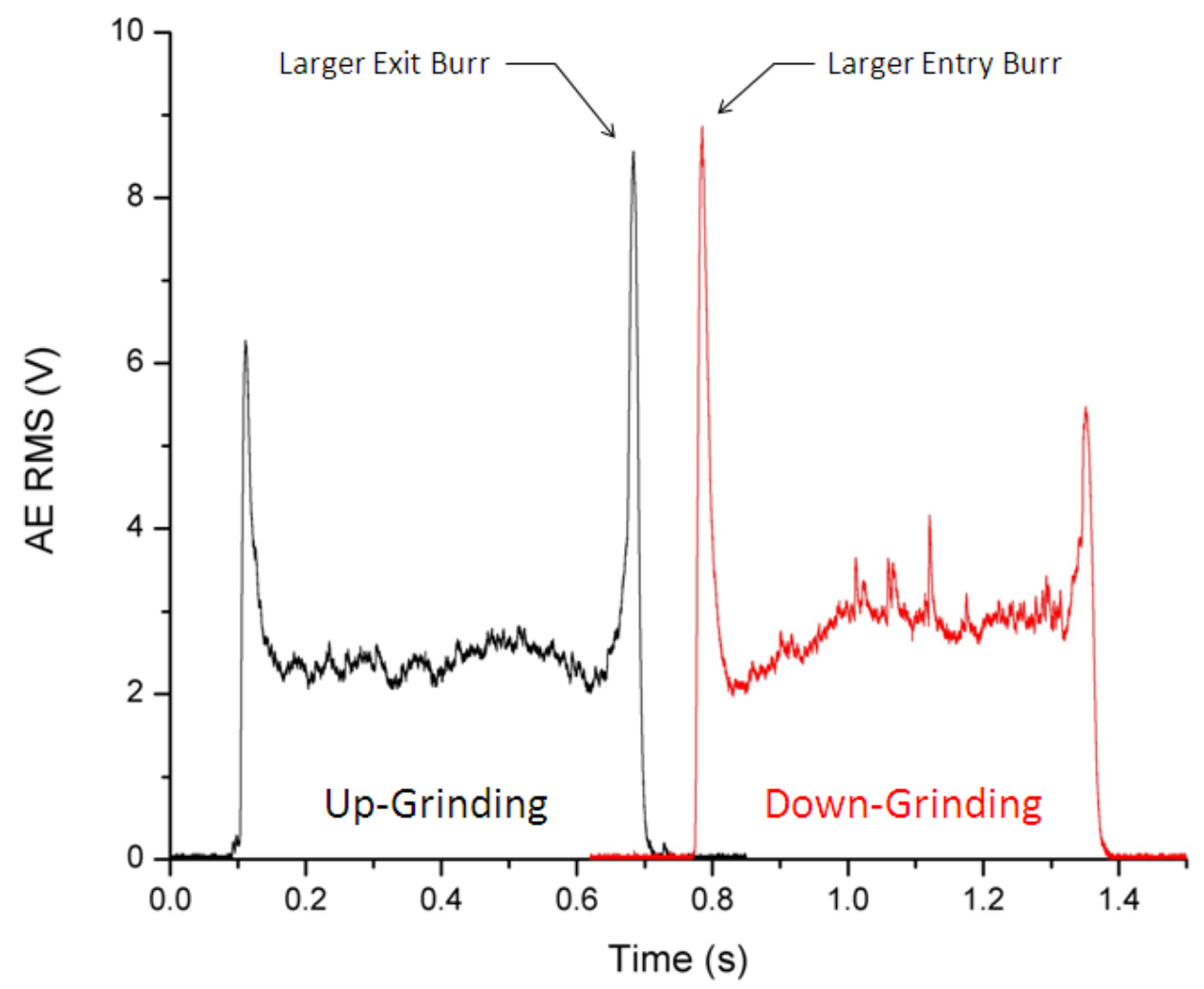

Figure 4.3: Common AE RMS responses, comparing up-grinding to down-grinding.

The general trend shows that up-grinding yields a larger AE exit spike, whereas downgrinding results in a larger entry spike. This is a consequence of burr formation, as exit burrs are significant during up-grinding, while entry burrs are dominant during down-grinding. The interaction between the rotation of the grinding wheel and direction of the workpiece feed influences burr formation, and thereby has a corresponding effect on the $\mathrm{AE}$ entry and exit 
spikes. This is demonstrated in Figure 4.4, as it can be seen how the combination of wheel rotation and workpiece direction influences the way in which the workpiece edges are sheared.

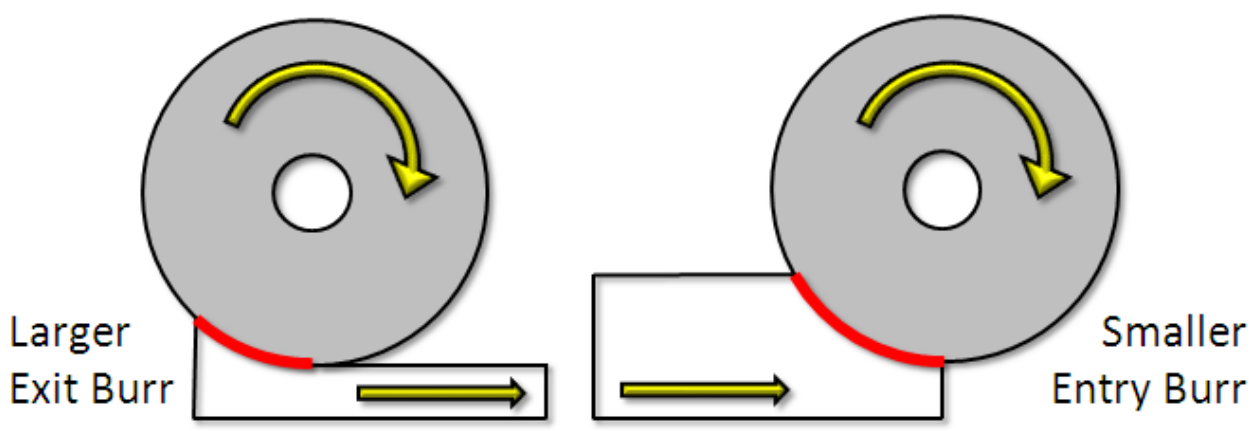

Up-Grinding

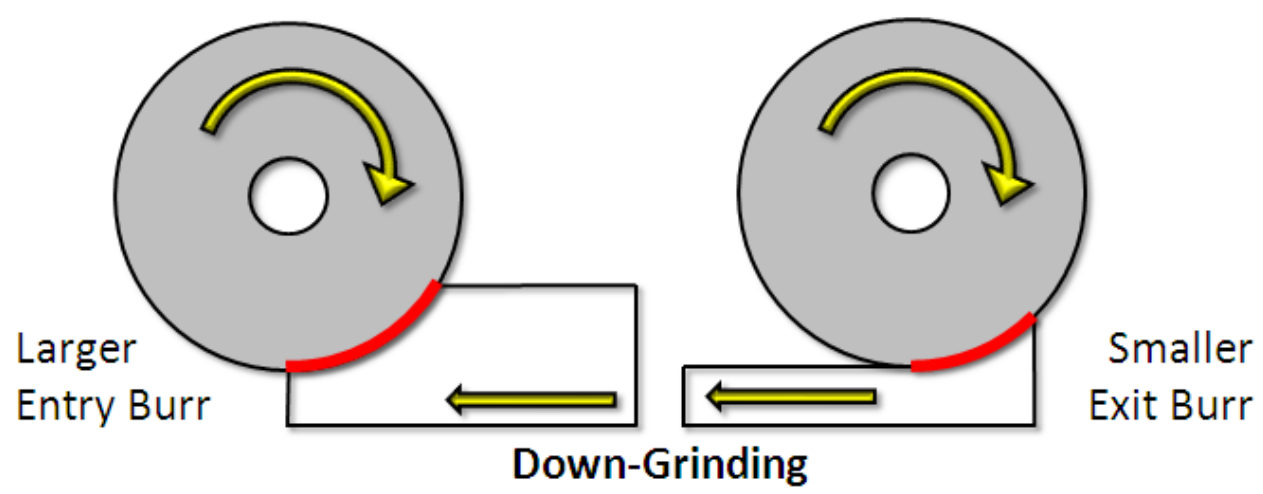

Figure 4.4: Illustration of the effect of workpiece entry and exit regions during up and down-grinding on burr formation. Figure is not drawn to scale.

The relative effects of wheel rotation and workpiece feed direction are shown in Figure 4.4. In the case of up-grinding, as is illustrated, the workpiece exit will yield the largest burr as both the rotation of the wheel and disengagement of the workpiece promote material flow away from the exit edge. Conversely, the entry edge was found to have the smallest burr, as the two engagement velocities combine to promote material flow into the workpiece itself, minimizing the burr formation along the entry edge [20]. The opposite is true for downgrinding.

The larger AE RMS spikes shown in Figure 4.3 attributed to the additional plastic deformation associated with the greater burr formation along these workpiece edges. Additional 
sources of increased acoustic emission may come as a result of elevated workpiece temperature within the burrs as discussed by Aurich et al. [21], and will be investigated in a later section.

\subsubsection{Effect of Coolant Application}

The amount of energy that is converted to heat during the grinding process is significantly higher when compared to other forms of traditional machining. The use of coolant therefore becomes vital to provide lubrication and decrease the temperatures within the grinding zone that can exceed $700^{\circ} \mathrm{C}$. Coolant application generally helps in producing a better surface finish while also decreasing the effects of wheel wear. A simple comparison of the AE RMS signal is shown in Figure 4.5 to show the effect of coolant application during otherwise identical surface grinding experiments.

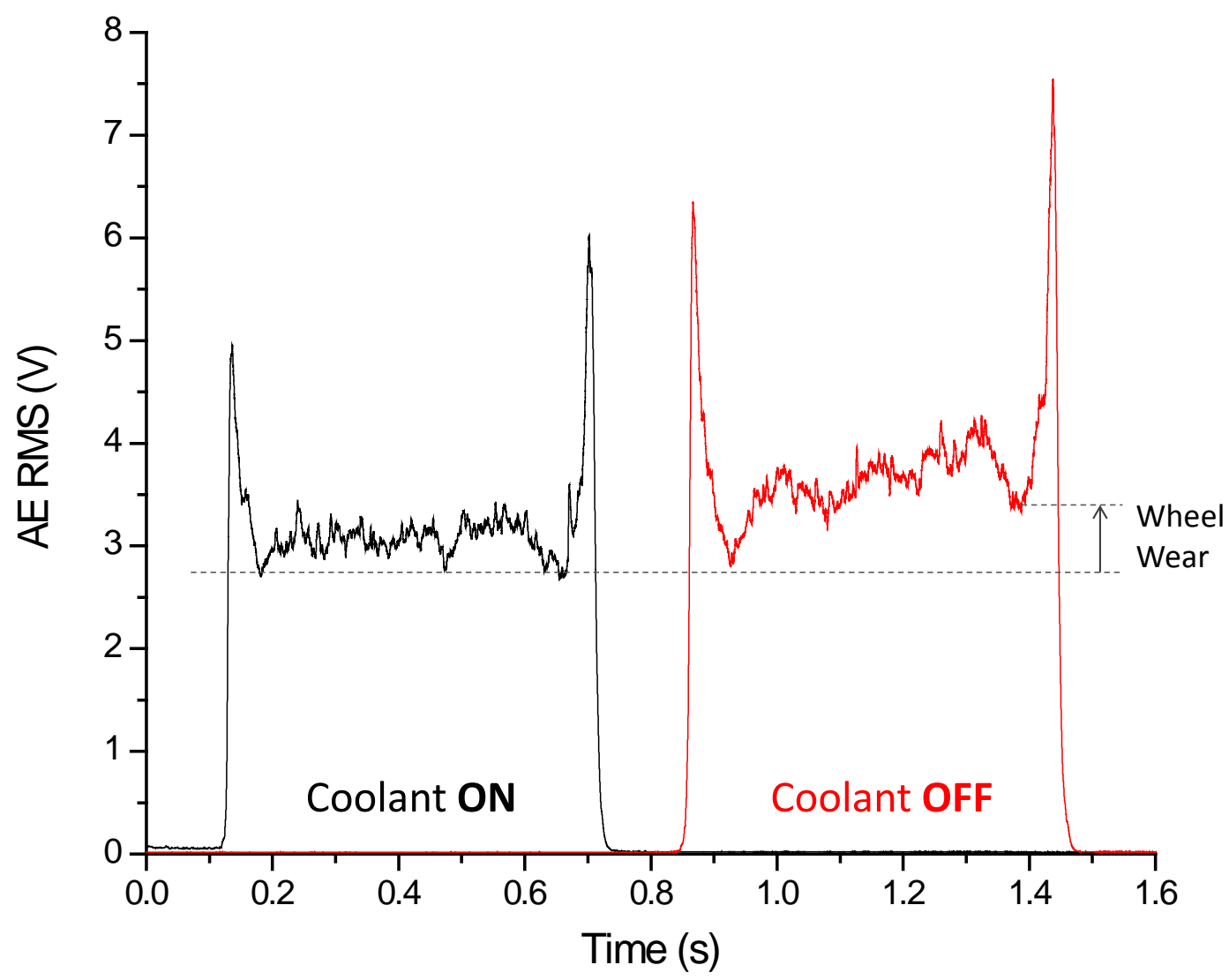

Figure 4.5: AE RMS signals comparing the effect of coolant during up-grinding. 
The result shows that there is a slight upward shift in the entire AE signal when grinding without coolant. This seems to be a consequence of wheel wear. The surface of the grinding wheel was inspected following the dry grinding experiment, and it clearly showed a dark glazed wear band along the circumference of the wheel. It was evident that when a coolant was not used, the wheel wear during an individual grinding pass was significantly higher than when coolant was applied, concluding that a worn wheel increases the AE response.

A similar AE response is seen in Figure 4.3, referring to an individual down-grinding signal. As discussed in Section 1.1, the grinding wheel enters the cut at the maximum chip thickness in the case of down-grinding. This has an increased effect on wheel wear progression when grinding brittle materials as there is greater initial impact between the abrasive grains and the workpiece [33]. The resulting increase in grain fracture and corresponding wheel wear is captured by the AE RMS signal in a similar manner to that of the dry grinding experiment shown in Figure 4.5. Additionally, the effect of wheel wear is also noticeable in Figure 4.1, wherein the general level of the continuous segment of the $\mathrm{AE}$ signal can be seen to shift to higher magnitudes with each successive grinding pass.

The relative entry and exit spike amplitudes remain similar for both cases of coolant application, which indicates that coolant does not significantly influence the AE spike phenomenon. All experiments were therefore performed using coolant as is the common industrial practice with a view to controlling wheel wear. 


\subsubsection{Effect of Workpiece Geometry}

Both up-grinding and down-grinding have been investigated, and it has been found that up-grinding exit burrs tend to be the most significant. This agrees with the literature which found that up-grinding exit burrs are larger than those produced during down-grinding [19] as well as being "by far the largest in size" [21]. The previous results have shown that burr formation does in fact have an effect on the amplitude of the AE entry and exit spikes. However, in order to determine if burr formation is indeed the dominant factor behind AE spike existence, the signal should be investigated under conditions that either intensify its growth or avoid burr formation altogether.

Keeping the grinding parameters constant, the workpiece edge geometry was changed in order to manipulate the formation of grinding burrs. Several HSS workpieces were prepared to correspond to a range of entry and exit angles, in addition to the $90^{\circ}$ baseline case. The workpiece geometry convention has been presented previously presented in Figure 3.3.

The workpiece edges were investigated following surface grinding experiments along with the respective acoustic signal. Burr formation was found to be the greatest on the $135^{\circ}$ workpiece, but was reduced to an insignificant level on the $45^{\circ}$ and $5^{\circ}$ samples. This agrees with Kawamura's work on the effect of workpiece geometry on burr size [19], and seems to translate directly into the acoustic signals, as both entry and exit spike amplitudes increased with increase in the workpiece edge angle. Figure 4.6 shows the respective AE RMS signals for each of the specified workpiece geometries using the grinding parameters listed in Table 3.1. 


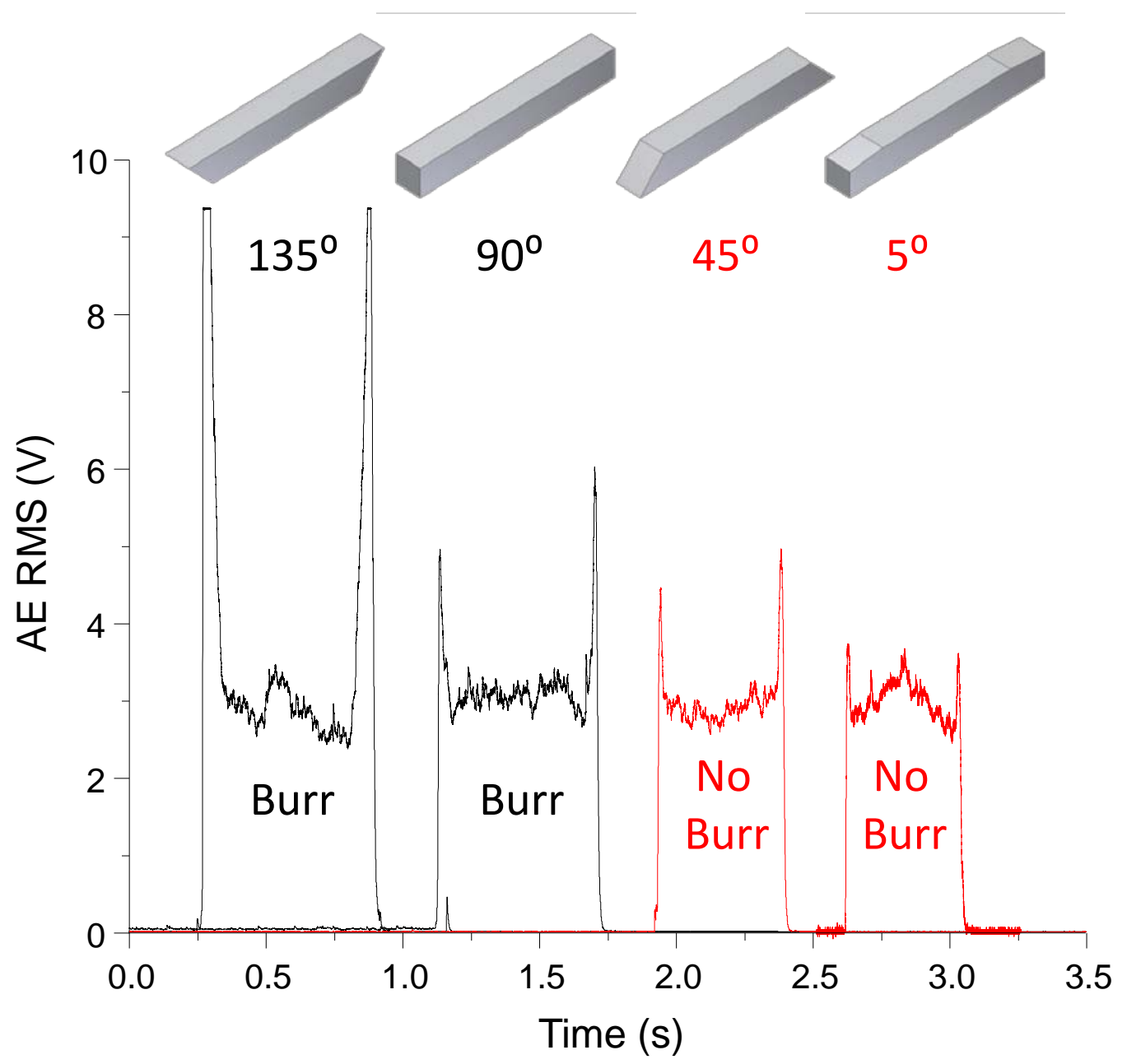

Figure 4.6: Comparison of AE RMS signals as a result of only changing workpiece edge geometry. Burrs were observed only in the $135^{\circ}$ and $90^{\circ}$ edge workpieces.

It is clearly evident that workpiece edge geometry has a very significant effect on the occurrence of AE entry and exit spikes. The spike amplitude attains values over $300 \%$ of the continuous region for the $135^{\circ}$ workpiece, decreasing down to an almost a negligible amount for $5^{\circ}$. In order to prove the hypothesis of $\mathrm{AE}$ spikes being influenced by burr formation, their interdependence was investigated.

Having documented the strong effect of workpiece edge geometry on burr formation and AE RMS spikes, a new goal was set to determine if all four spike/burr combinations were 
possible: situations with AE spikes and burrs, spikes but no burrs, no spikes but burrs, and neither spikes nor burrs. If all situations above were found to exist, it would prove that factors other than burr formation also cause the AE spikes. Several variations of workpiece edge geometry were investigated for this series of experiments, including some with compound angles.

One of the more interesting findings came when a single workpiece was used to yield three completely different results. When the $45^{\circ}$ workpiece is simply rotated about its longitudinal axis, the edge geometry can represent three distinct orientations. This introduces a secondary angle which controls the engagement of the wheel across the workpiece width during entry and exit, as illustrated in Figure 4.7.

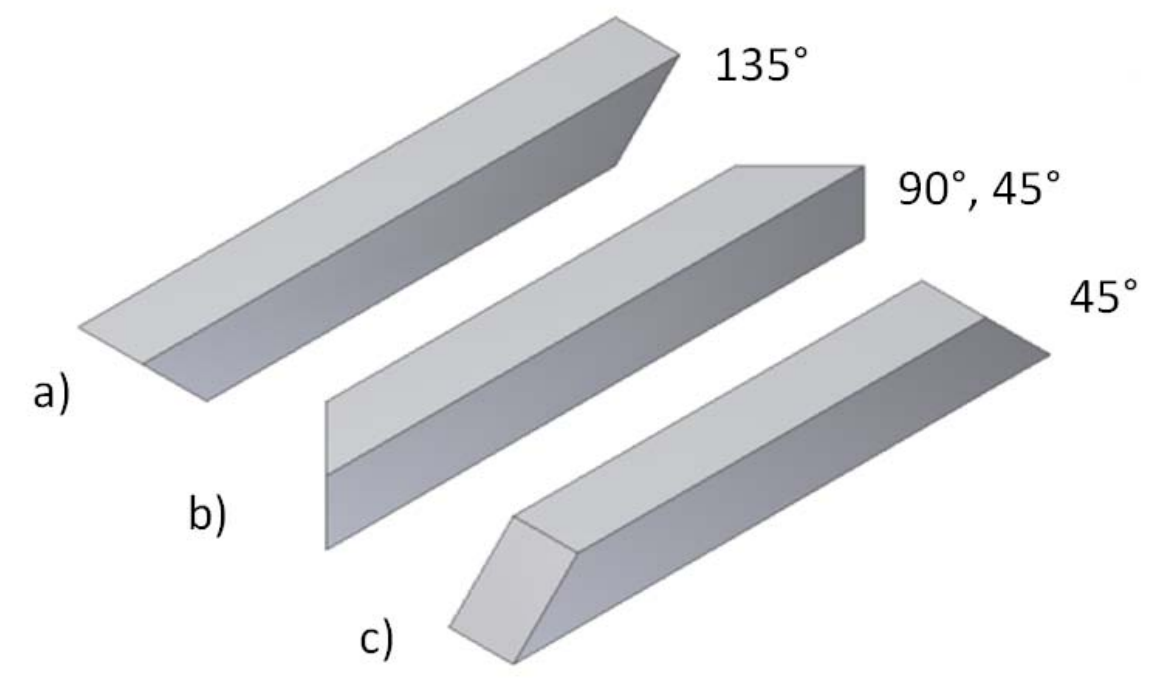

Figure 4.7: Illustration of three different edge geometries using the same workpiece. A secondary angle is introduced in orientation b) corresponding to the degree of gradual grinding width during entry/exit.

The AE responses of orientation a) and c) have been previously shown in Figure 4.6, however, a new result is achieved when introducing the workpiece orientation shown in Figure $4.7 \mathrm{~b}$ ); the case of burr formation without AE spikes. Figure 4.8 compares all of the new 
investigated cases involving workpiece geometry, with indication of when burrs were observed. The same grinding parameters were used as listed in Table 3.1, with each pass following wheel dressing and workpiece deburring.

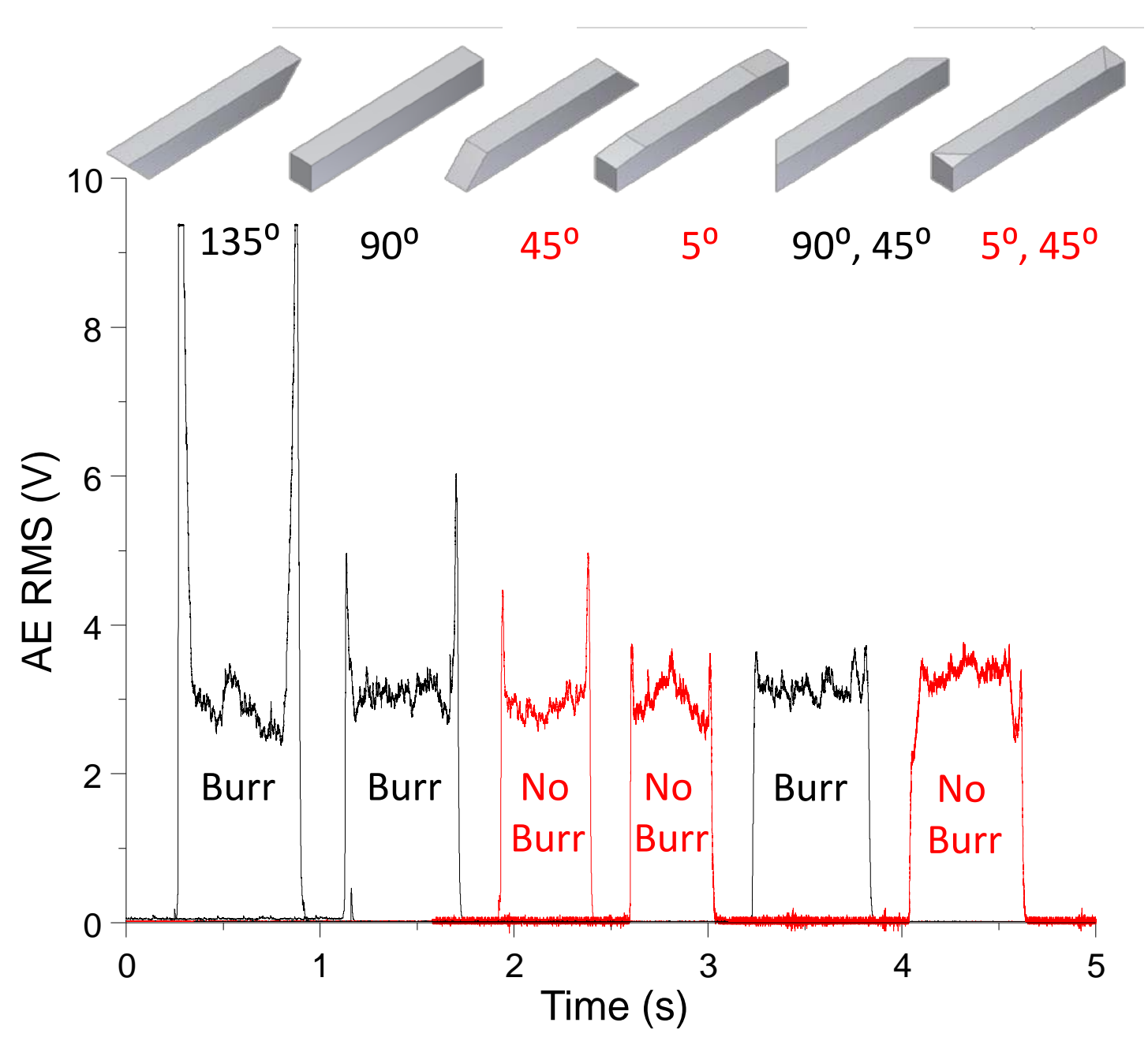

Figure 4.8: Comparison of AE RMS signals as a result of only changing workpiece edge geometry. All four scenarios of grinding burr/AE spike existence are presented.

It is evident that burr formation is dependent on the primary workpiece entry and exit angles and not the secondary angles that introduce the grinding width gradually. The most significant burr growth occurs at primary angles that are greater than $90^{\circ}$ which strongly agrees 
with Kawamura's finding of rapidly decreasing burr heights below this edge angle [19]. Table 4.1 summarizes the results for the interdependence between AE spikes and burr formation.

Table 4.1: Scenarios of AE spike existence and grinding burr formation

\begin{tabular}{|c|c|c|}
\cline { 2 - 3 } \multicolumn{1}{c|}{} & \multicolumn{1}{c|}{ AE Spikes } & No AE Spikes \\
\hline Burr Formation & $135^{\circ}, 90^{\circ}$ edge geometry & $45^{\circ}$ gradual engagement \\
\hline No Burr Formation & $45^{\circ}$ edge geometry & $<5^{\circ}$ edge geometry, \\
& & $5^{\circ}: 45^{\circ}$ compound angle \\
\hline
\end{tabular}

All four distinct AE spike/burr combinations are possible, indicating that the AE entry and exit spike phenomenon is not solely caused by burr formation. Burr formation however does indeed influence the amplitude of the AE entry and exit spikes as was shown in Sections 4.1.1 and 4.1.2.

Returning to Figure 4.8, the wide range of acoustic signals that can exist is clearly evident. Even though identical surface grinding tests were performed on the same workpiece material, the AE entry/exit spikes vary so greatly as a result of simple geometrical changes. It has been found that the edge geometry does influence burr formation, but this does not directly translate into AE spikes. Entry and exit spikes do not just randomly occur, nor can they be simply classified as the same for every case. The AE spike phenomenon is in fact largely dependent on the workpiece engagement/disengagement geometry, but the fundamental cause of its behavior is yet to be determined.

In this context, the duration of an AE exit spike was compared to the burr length formed at the exit edge of the workpiece. Since the AE RMS signal is plotted as a voltage waveform versus time, and the workpiece feedrate is known, the corresponding distance can be calculated. Even for the most conservative values, it was found that the AE exit spike length corresponded 
to a minimum distance of approximately $5 \mathrm{~mm}$ for the $90^{\circ}$ workpiece. When compared to the maximum measured burr length of approximately $100 \mu \mathrm{m}$, the lengths differed by at least a factor of 50. Even though this result failed to link the AE spike to burr length, it was expected due to the previous finding that burr formation is not solely responsible for AE spike behavior. However, it was this initial attempt of examining the AE spike interval that introduced a more indepth investigation of the AE entry and exit spike durations.

An AE RMS signal corresponding to $90^{\circ}$ workpiece edge geometry was initially considered, and the average entry and exit spike lengths were calculated to be approximately $6 \mathrm{~mm}$. This finding was of immediate interest, as typical AE burst-type signals only last 2-3 ms which would translate to a length of only $0.5 \mathrm{~mm}$ at the same workpiece feedrate. It can be therefore be stated that the AE spikes occurring at workpiece entry and exit are not simply caused by a single AE burst event, but are sustained over a certain length of time. This entry and exit length of $6 \mathrm{~mm}$ was studied in order to determine what was possibly occurring in this region of the workpiece, and why it was causing an increase in the acoustic signal.

The following section will discuss the mechanical interaction between the grinding wheel and the workpiece along with an investigation of the AE entry and exit spike regions by systematically varying the wheel depth of cut. 


\subsubsection{Effect of Wheel Depth of Cut}

Before attempting to identify a correlation between AE spike duration and the grinding entry/exit regions, an understanding of the grinding mechanics is essential. The grinding parameters that allow for geometrical modeling of the process are the diameter of the wheel, the wheel depth of cut, and the table feedrate. This information is listed in Table 4.2.

Table 4.2: Fundamental grinding parameters

\begin{tabular}{|c|c|}
\hline Parameter & Value \\
\hline Grinding wheel diameter* $^{*}$ & $355 \mathrm{~mm}$ \\
\hline Depth of cut & $10 \mu \mathrm{m}$ \\
\hline Workpiece feedrate & $10.16 \mathrm{~m} / \mathrm{min}$ \\
\hline
\end{tabular}

* It should be noted that the grinding wheel diameter continually decreases following dressing, and so this varying value was recorded before each set of experiments.

These basic values can be used to model the surface interaction between the circular grinding wheel and the flat workpiece based strictly on geometry. Calculating the geometric contact length is a common method used to gain insight into the grinding process. Figure 4.9 illustrates the required physical parameters and indicates the geometric contact length, $l_{g}$, which can be calculated using equation 4.1,

$$
l_{g} \approx \sqrt{d D}
$$

where $d$ is the depth of cut and $D$ is the diameter of the wheel. 


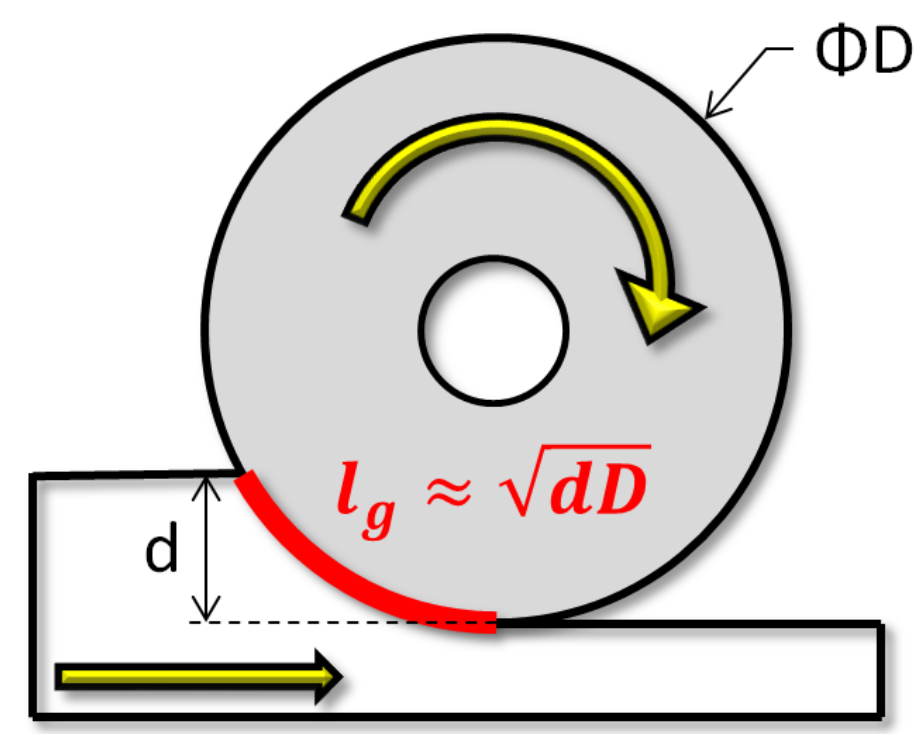

Figure 4.9: Factors contributing to the geometric contact length. Not to scale.

It can be seen that this calculation is purely dependent on geometry, assuming the ideal case of a perfectly circular rigid wheel. Considering that the diameter of the wheel is so large in comparison to the depth of cut, and that its value remains relatively unaffected in a single grinding pass, changes to the depth of cut are of most significant affect to the geometric contact length.

As stated earlier, for the data collected from the $90^{\circ}$ workpiece, the spike duration corresponds to an approximate distance of $6 \mathrm{~mm}$ from the time of first wheel contact. Equation 4.1 was used to calculate $l_{g}$ to be approximately $1.9 \mathrm{~mm}$ for this case. Therefore, the calculated geometric contact length corresponds to roughly three times less as low as the measured AE spike duration. This result may seem to be of little consequence in the first instance, but in fact is quite interesting considering that the literature $[32,34,35,36]$ reports the actual contact length between the grinding wheel and the workpiece to be typically two to three times the geometrically calculated value mainly due to elastic deformation in the contact zone. 
In light of these results, a new hypothesis was proposed, stating that the duration of the $\mathrm{AE}$ entry and exit spikes represents the actual contact length between the grinding wheel and workpiece. In order to verify this, a new set of experiments was designed in which the geometric contact length could be varied in a controlled manner. Referring to equation 4.1, the geometric contact length can be manipulated by altering either the depth of cut or the wheel diameter. As the realistic solution is to vary the depth of cut, five otherwise identical surface grinding experiments were performed on the $90^{\circ}$ HSS workpiece, each with a different cutting depth. Depths were incremented by $10 \mu \mathrm{m}$ from the baseline case up to a maximum of $50 \mu \mathrm{m}$, with the experiments conducted in a random order. The AE RMS results are shown in Figure 4.10.

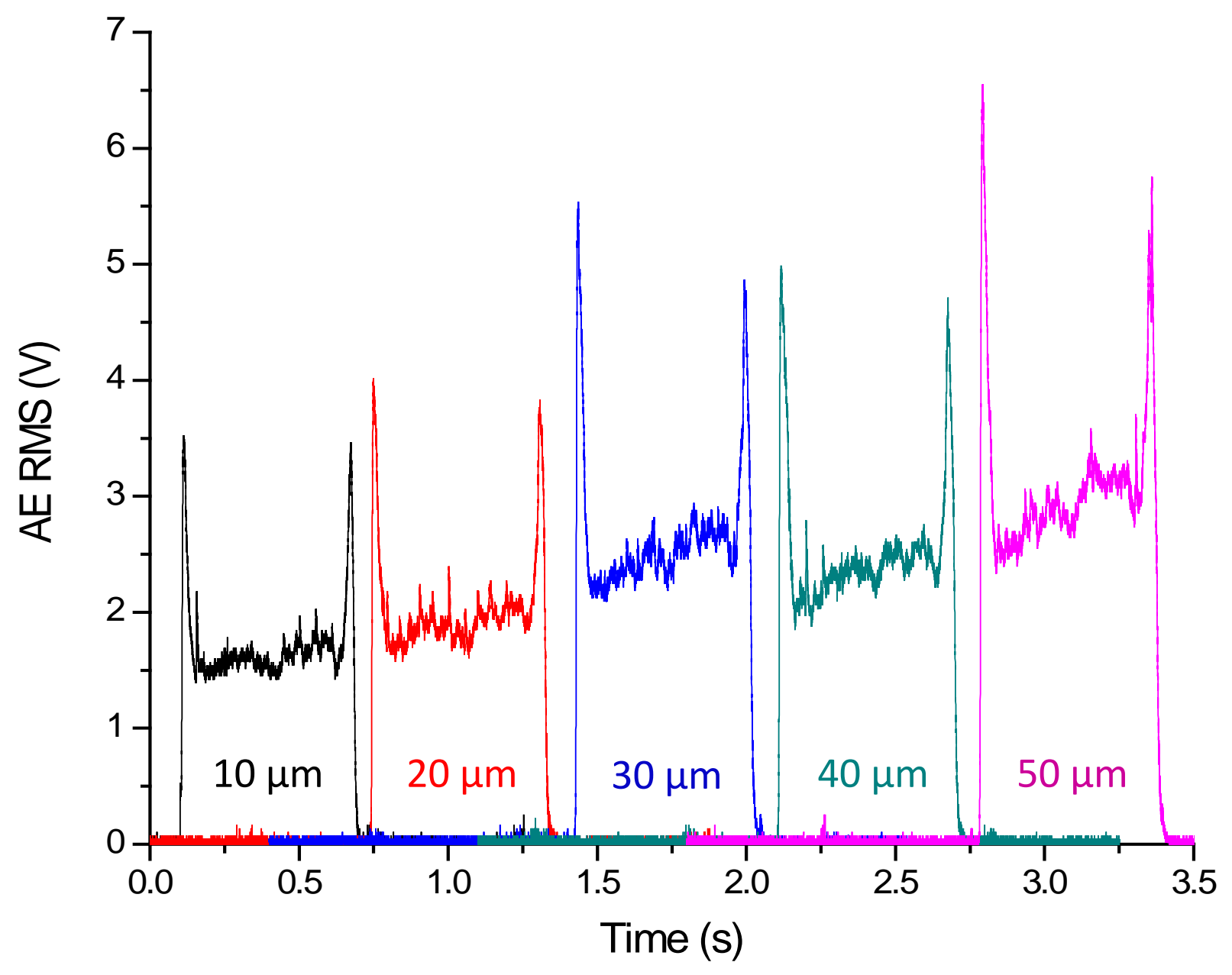

Figure 4.10: AE RMS comparison at varying depths of cut. 
There are several noteworthy trends to discuss in these results. The most relevant to the hypothesis at hand are the $\mathrm{AE}$ spike durations at each depth of cut in comparison with the calculated geometric contact length. However, the first task in data analysis involved calculating the corresponding time-lengths of each acoustic emission spike. In order to eliminate the subjectivity in identifying when the $\mathrm{AE}$ spikes end, the time at which the first derivative of the AE RMS signal crosses zero the second time was considered, as demonstrated in Figure 4.11.

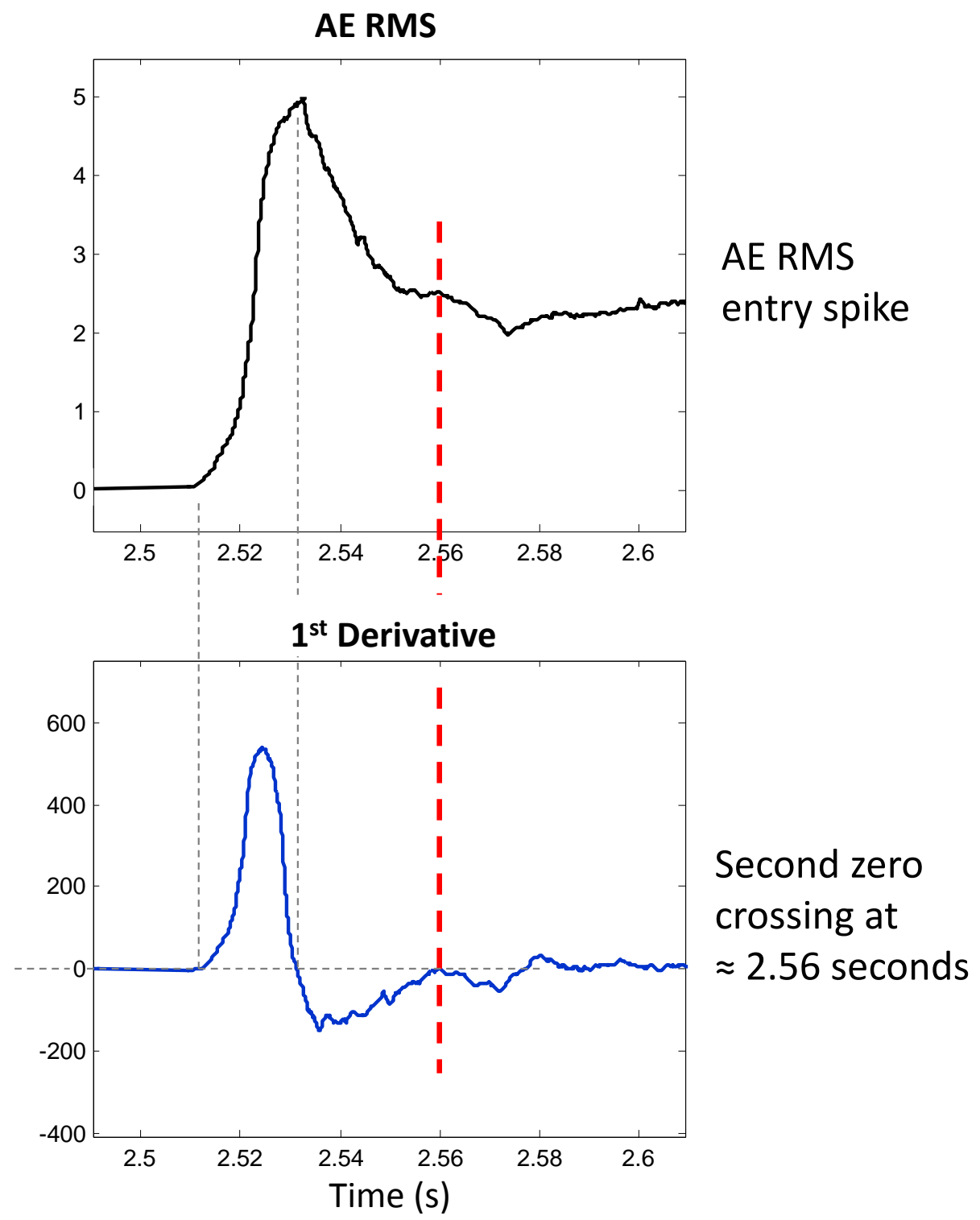

Figure 4.11: Comparison of an AE RMS entry spike and its derivative. The instance when the derivative crosses zero is highlighted, corresponding to the end of the AE spike region. 
Using the data from Figure 4.10, the time durations of each AE entry and exit spike were thus calculated and converted to a linear distance by considering the workpiece feedrate of $10.2 \mathrm{~m} / \mathrm{min}$. The results are plotted in Figure 4.12 which shows the calculated contact length at different depths of cut. Values corresponding to the geometric contact length as well as $2 x$ and $3 \times$ the geometric contact length are also plotted for a visual comparison.
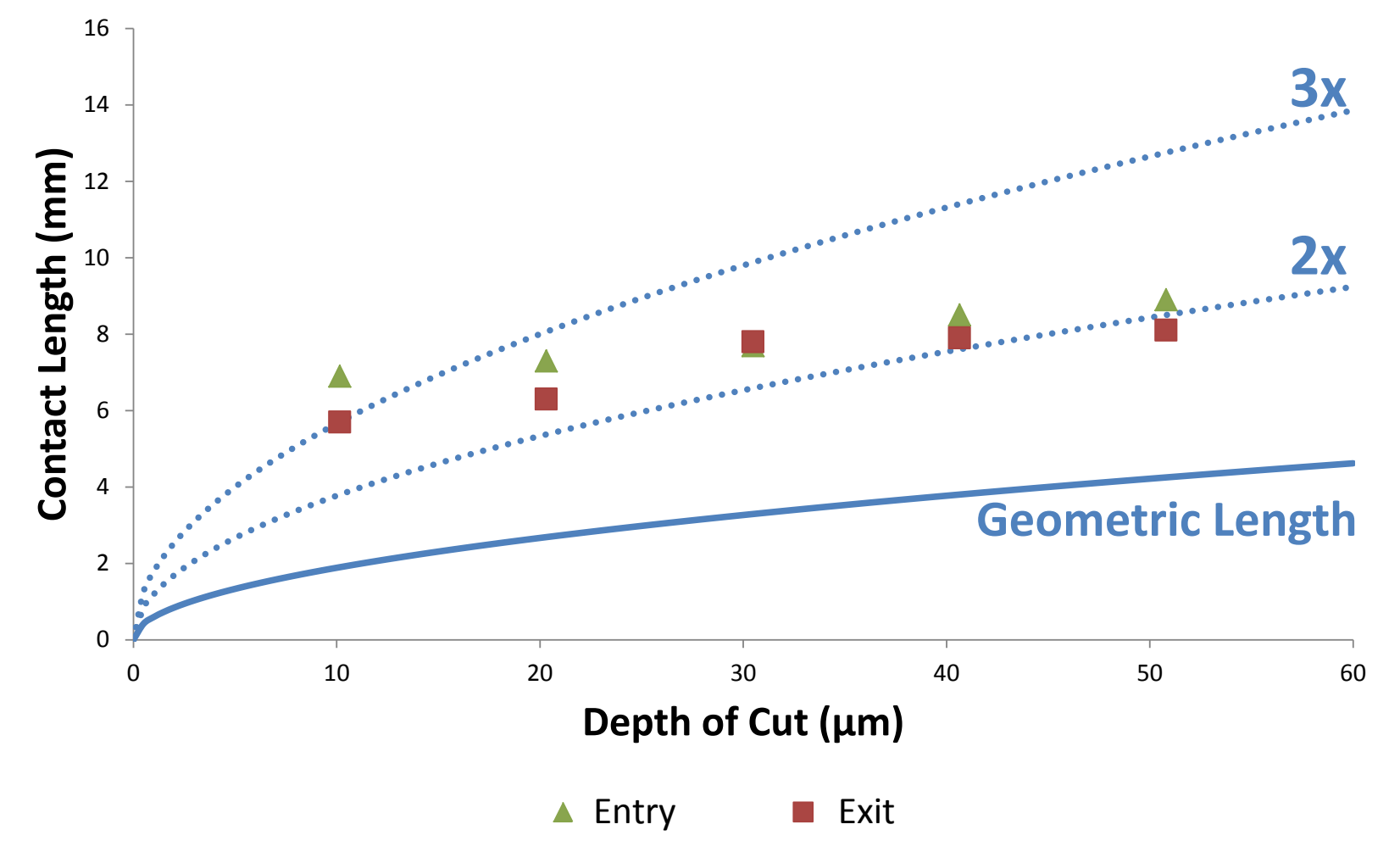

Figure 4.12: Calculated wheel/work contact length vs. depth of cut for up-grinding.

This result is exciting as it validates the hypothesis, and is in conformance with trends found in the literature $[32,34,35,36]$ shown in Figures 2.16 - 2.18. It can be seen that the contact length increases with an increase of the depth of cut, and is between two to three times the geometric contact length. These results are indeed of great practical significance as accurately measuring the contact length is critical in correctly predicting grinding burn and grinding zone temperatures. 
It is perhaps of interest at this time to discuss some features portrayed in the data shown in Figure 4.10. When looking at the AE signals, especially those at a higher depth of cut, the continuous region of signal seems to be on an increase during grinding. The most logical reason for this result appears to be wheel wear. As mentioned in Section 4.1.3, the entire AE RMS signal becomes elevated during tests with a loaded and/or dull grinding wheel. In the case of Figure 4.10, deeper cuts actually resulted in the workpiece surface to burn and the grinding wheel to wear significantly during a single grinding pass. The grinding wheel was also observed to have a dark glazed band along its surface immediately following an individual test at cutting depths greater than $30 \mu \mathrm{m}$. This mimics the result shown in Figure 4.5 when no coolant was applied. As the grinding wheel becomes worn, grinding forces increase, workpiece surface quality reduces, and temperatures rise as a result of greater friction. This causes acoustic emission levels to increase.

Another noteworthy observation regarding Figure 4.10 refers to grinding burn and will therefore be discussed later in Section 4.2.3.

\subsubsection{Contact Length Verification}

Current methods of calculating the actual contact length in surface grinding have been discussed in Section 2.4; all of which are tedious and destructive processes. The results that link AE RMS spikes to the actual contact length between the wheel and workpiece is novel, and can prove to be of remarkable significance. Accurate estimation of the contact length is crucial to modelling heat transfer in grinding. It is known that the actual contact length can range from $150 \%-300 \%$ of the geometric contact length, or even higher in some cases, and so this value becomes important when estimating grinding temperatures $[33,34,35,36]$. 
The preliminary results are promising, displaying trends identical to current contact length measurements; however, more experiments were performed to verify this. The results shown in Figure 4.12 were based on up-grinding. A similar set of tests was next performed in down-grinding in order to compare the results. The same $90^{\circ}$ workpiece edge geometry was used with the identical grinding parameters, and the results were analyzed in the same way. The entry and exit spike durations were calculated for each depth of cut, and again plotted against the depth of cut. Figure 4.13 shows these results superimposed over the previously calculated values referring to up-grinding.

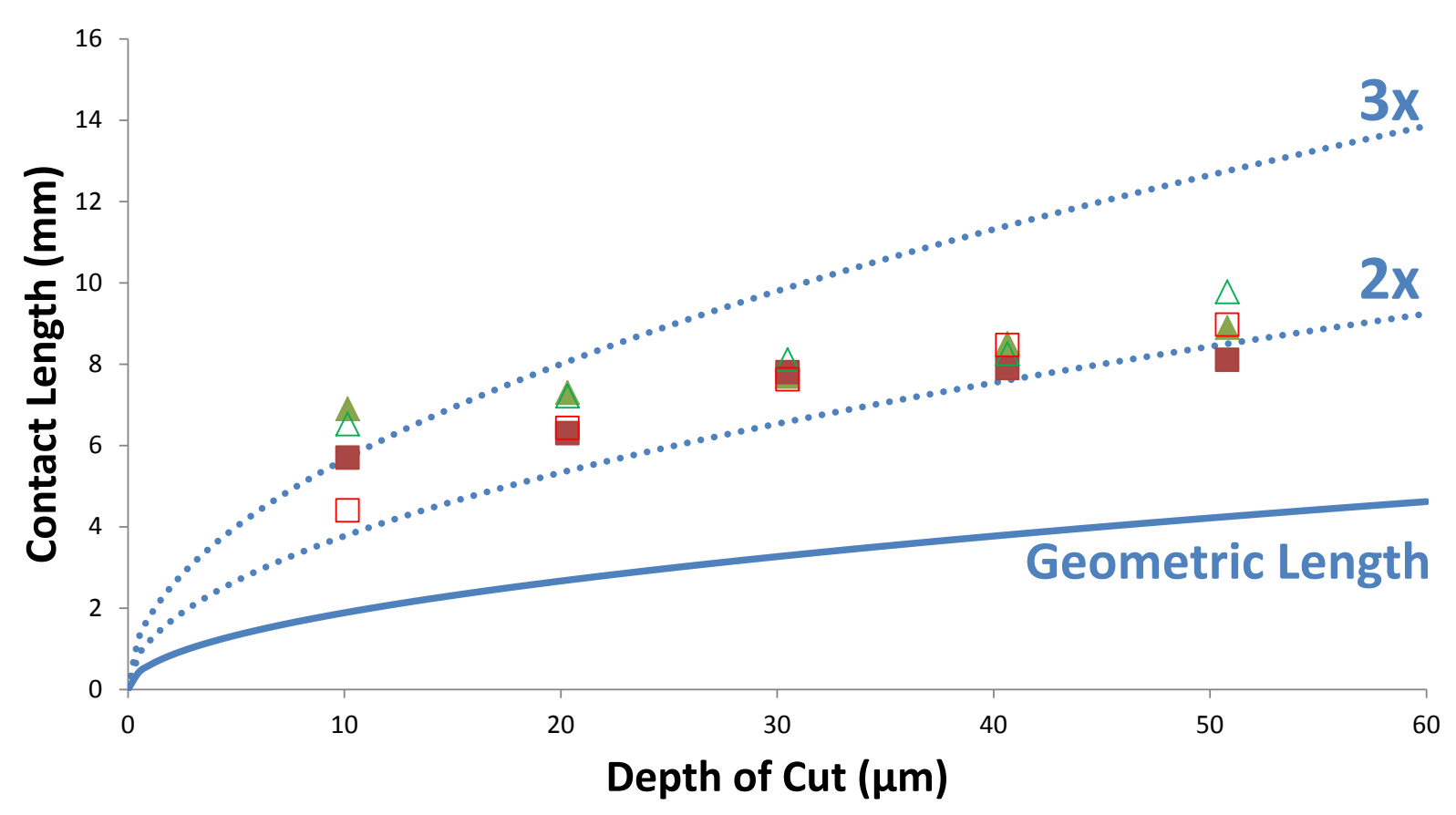

$\triangle$ Up Entry $\quad$ Up Exit $\quad \supset$ Down Entry $\quad \square$ Down Exit

Figure 4.13: Calculated wheel/work contact length vs. depth of cut for both up and down-grinding.

The results are shown to follow the same trend for both up and down-grinding, with $\mathrm{AE}$ spike length values ranging between $2 \times$ and $3 \times$ the geometric contact length. It is also interesting that the actual contact length to geometric contact length ratio decreases from three to two as the 
depth of cut increases. A likely reason for this occurrence can be based on the relationship between grinding force and the wheel depth of cut. Assuming a constant contact pressure [33], the applied force becomes proportional to the square root of the depth of cut. Since the force largely contributes to the amount of elastic deformation in the grinding zone, it indirectly influences the actual contact length. Therefore, as the depth of cut is increased, the force (and corresponding elastic deformation) increases proportional to its square root, resulting in the contact length ratio to slightly decrease. This trend is also reported in literature $[32,35,36]$, making this result even more significant and comparable to accepted contact length models. Literature [36] also reports that the difference in actual contact length between up-grinding and down-grinding is less than $8 \%$, and in light of measurement error, can be deemed insignificant.

The results indeed look favourable, but it must be reiterated that they are strictly based on a $90^{\circ}$ workpiece entry and exit geometry. Figure 4.14 illustrates wheel/workpiece interaction in order to visualize the initial grinding wheel engagement and understand how it relates to the $\mathrm{AE}$ spike.

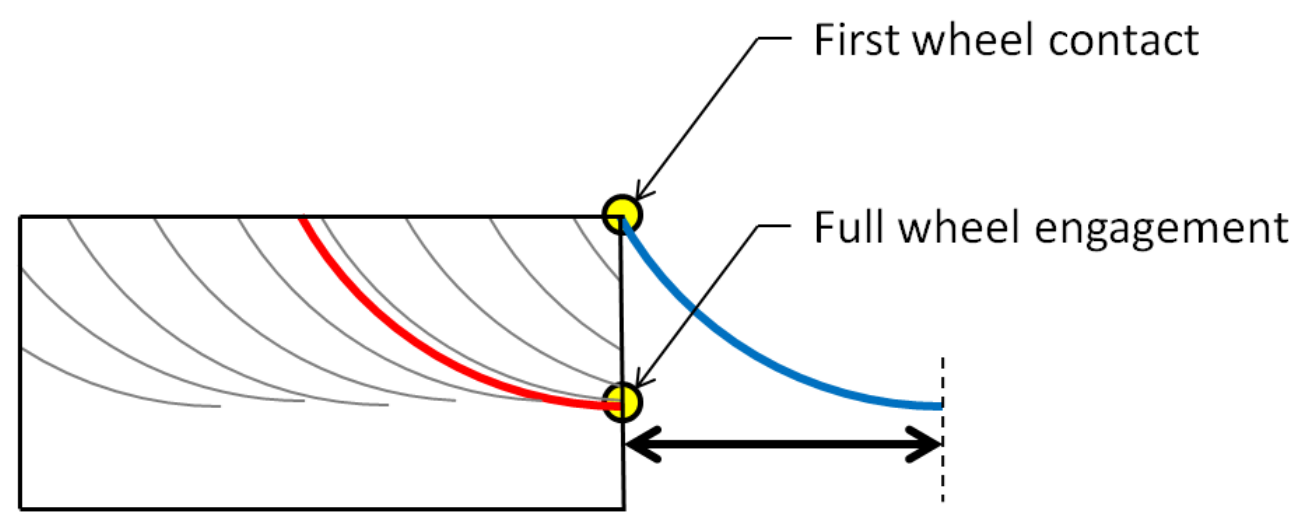

\section{Transition length for full wheel engagement}

Figure 4.14: Illustration of wheel/workpiece interaction at grinding wheel entry. 
The curves represent the regions of the grinding wheel that interacts with the workpiece as it is fed across the surface- - the contact length. The blue curve represents the instant of initial wheel contact and the red curve represents the moment when full wheel engagement is made. It must be noted that this figure is not drawn to scale, and that the actual diameter of the grinding wheel is much greater (approximately 3000 times greater) than the depth of cut. As a result of the relatively tiny length of the wheel in contact with the workpiece, the length of the curve can be approximated by its horizontal distance; the same horizontal distance as defined by the AE spike duration. This is illustrated in Figure 4.15.

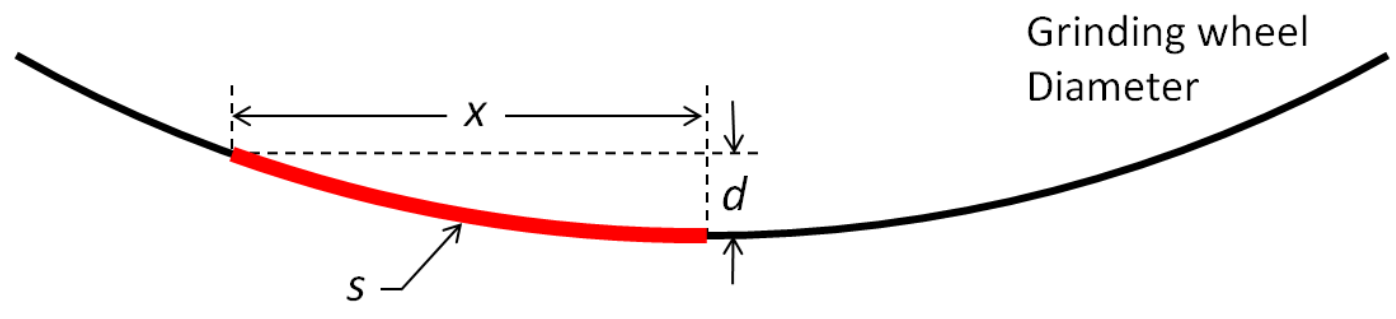

Figure 4.15: Geometric illustration of the grinding zone, not drawn to scale.

This simple illustration is an exaggeration of the grinding zone meant to demonstrate the relationship between the contact length arc and the distance represented by the AE spike duration. The horizontal projection of $s$ can be used to approximate its length when small values of $d$ are used compared to the diameter of the wheel. In the most extreme case, using a depth of cut of $50 \mu \mathrm{m}$, the calculated ratio of $s / x=1.00007$. This verifies the method of using the horizontal distance $x$ to approximate the contact arc $s$ under the given grinding parameters, meaning that the AE spike duration accurately represents the contact length of the wheel. Of course such an assertion is not valid for creep feed grinding processes that correspond to wheel depths of cut on the order of millimeters. 
However, knowing that the AE spikes exist at the moment of wheel entry and exit, it is wrong to assume that the spike duration simply translates into the actual contact length irrespective of workpiece geometry. Instead, the AE spike duration can be said to correspond to the transition distance between the point of first wheel contact and the moment full wheel engagement has been made. The $90^{\circ}$ entry and exit geometry yields the ideal situation for the contact length estimation, as the curves for first contact and full engagement share a common point as illustrated in Figure 4.14.

It may be noted that AE RMS spike durations should be affected by changes in workpiece entry and exit geometry. Figure 4.16 illustrates how the transition distance for full wheel contact length depends on workpiece geometry. In the case of up-grinding, full wheel contact is defined to first occur when the initial chip thickness is zero and the full depth of cut is reached. The blue curve represents the point where first wheel contact is made, while the red curve denotes the instance of full wheel contact. It can be seen how the transition length increases as the workpiece edge geometry changes.

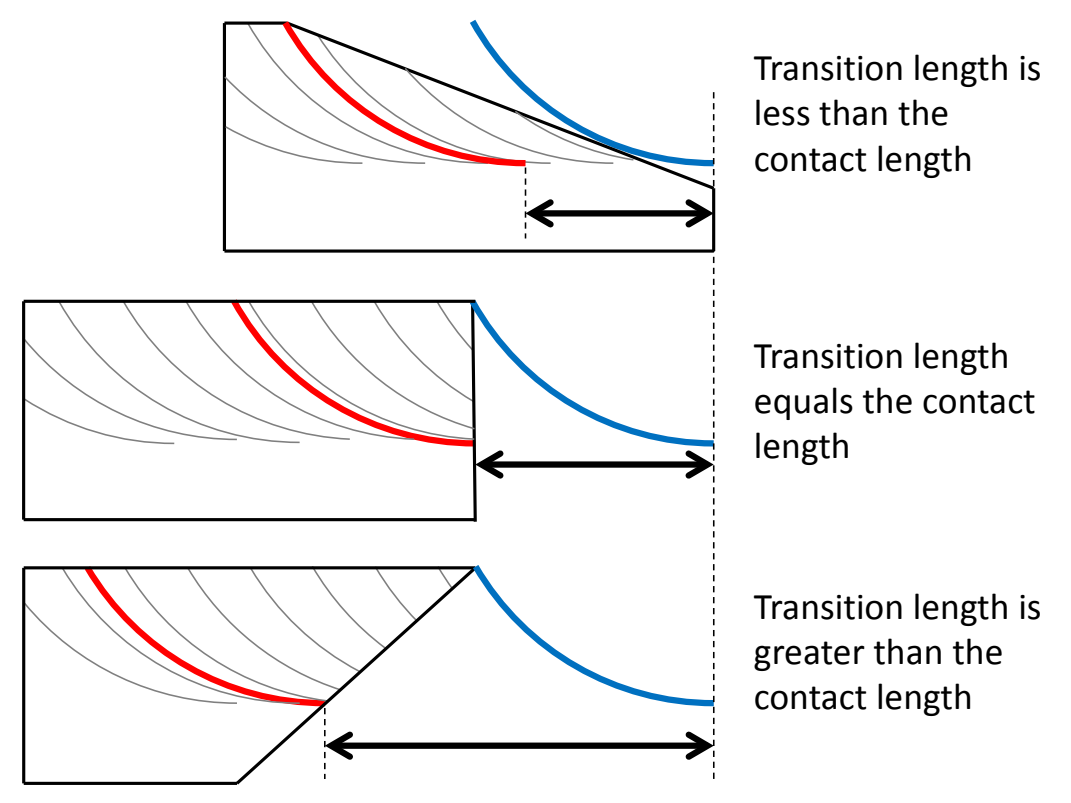

Figure 4.16: Demonstration of how the transition distance is dependent on workpiece geometry. 
In order to validate this concept in relation to the RMS signal, the AE spike durations were calculated for the $135^{\circ}, 45^{\circ}$ and $5^{\circ}$ workpiece edge angles and compared to the $90^{\circ}$ geometrical case. Figure 4.17 shows the results for the four different geometries. All tests were done using the baseline conditions in Table 3.1, following wheel dressing and workpiece deburring.

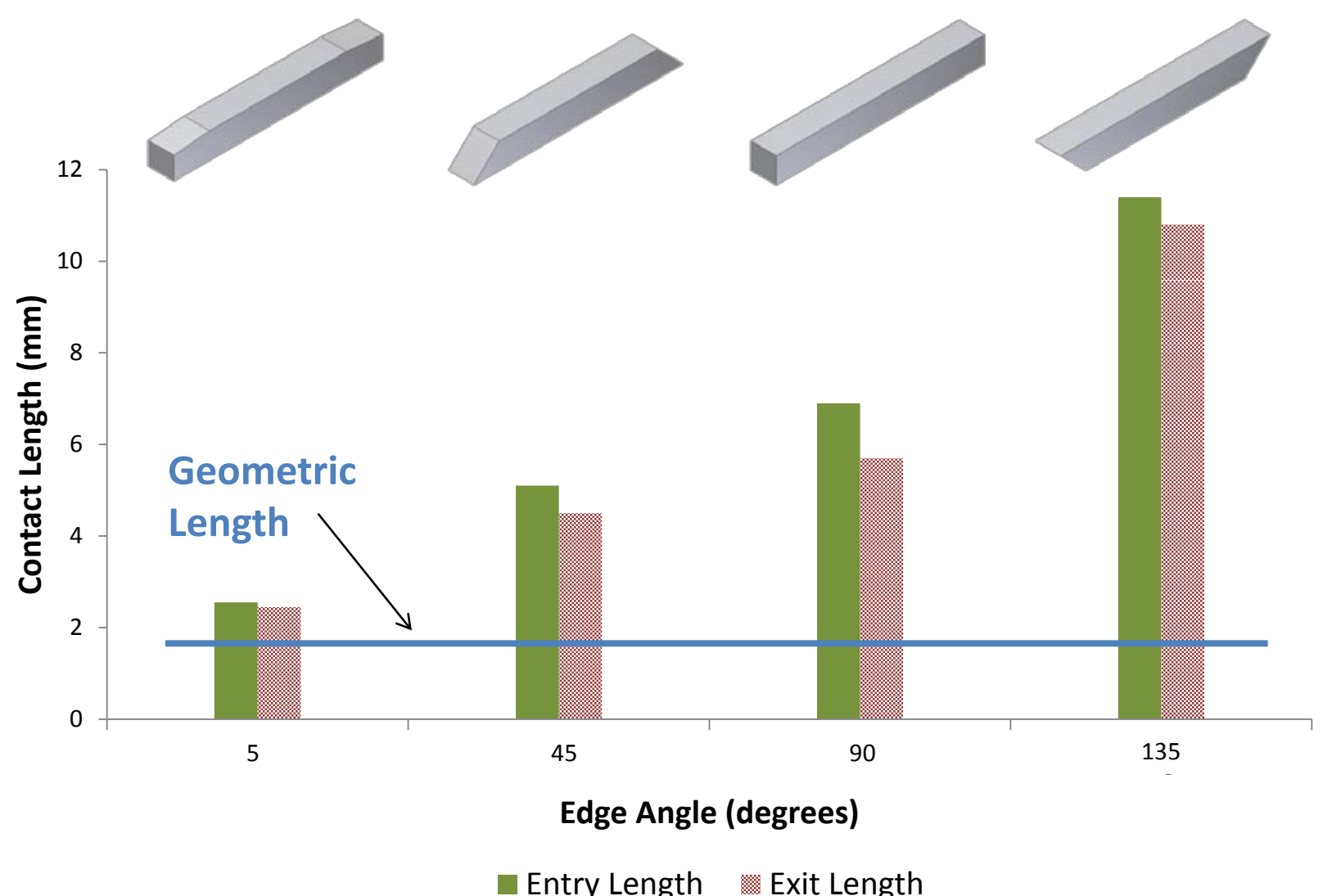

Figure 4.17: Experimental results of AE RMS spike duration as function of workpiece geometry.

It is evident that the $\mathrm{AE}$ entry and exit spike duration is significantly affected by workpiece geometry. The geometric contact length remains constant regardless of workpiece edge geometry, but the transition length for full wheel engagement/disengagement is what differs here. Therefore, in order for the proposed contact measurement technique to hold true, the AE spike durations taken from $90^{\circ}$ entry or exit angles must be used. 
To further assess the validity of the proposed technique for contact length measurement, the help of colleagues from RWTH Aachen University in Germany was enlisted. Researchers were advised of the basic contact length experiments to perform, but the method for estimating the $\mathrm{AE}$ spike durations and the result were not discussed beforehand. The outcome of their experiments is shown in Figure 4.18.

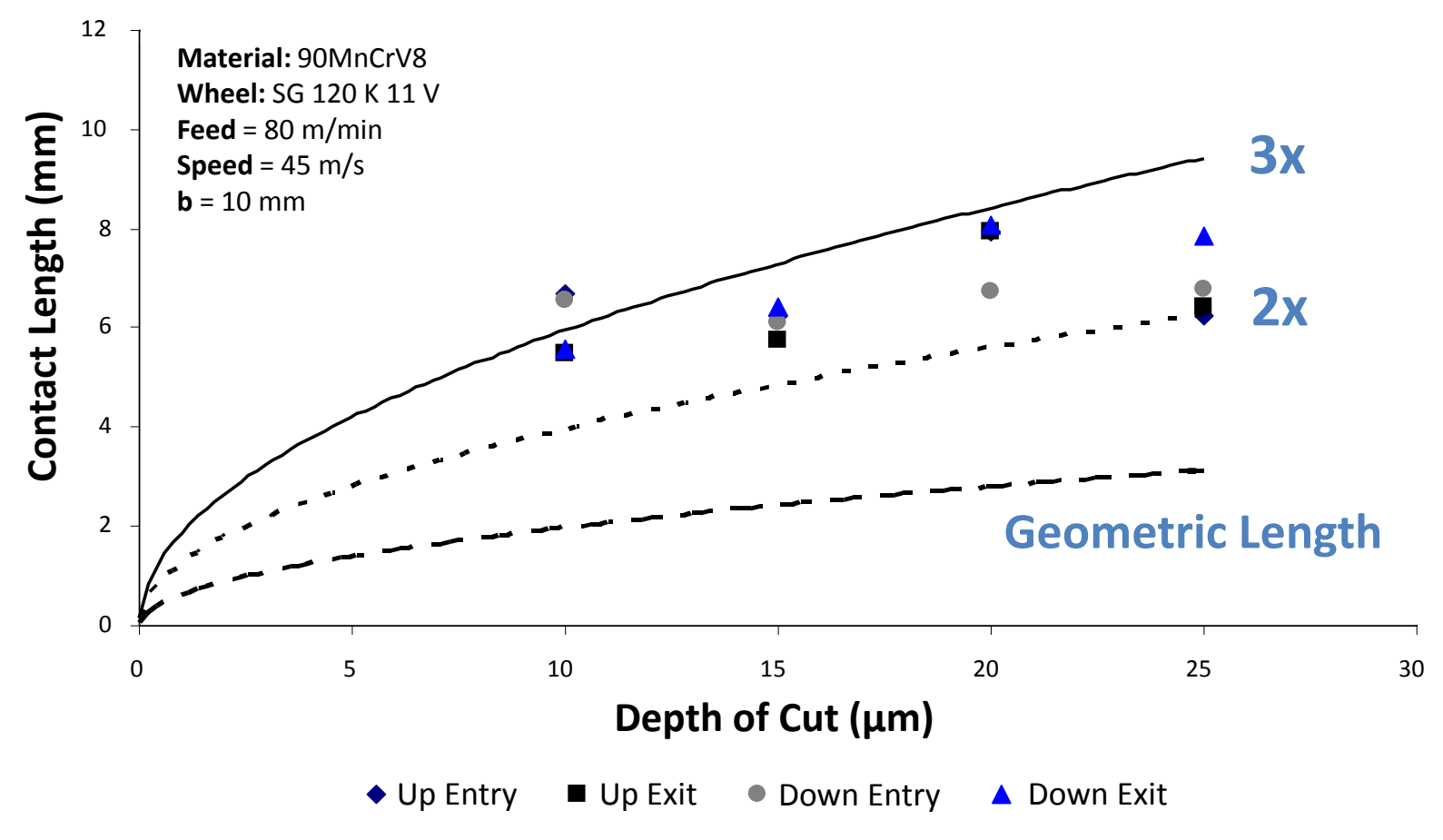

Figure 4.18: Contact length measurement results obtained from RWTH Aachen University.

It must be stressed that completely different machines, data acquisition systems, sensors, grinding wheels, workpieces, and grinding parameters were used. The AE RMS signals were analyzed by researchers in Aachen with no explicit instructions. The results are incredibly similar to those that were collected locally, as shown in Figure 4.13. This proves that the method of using AE spikes to estimate the actual grinding contact length is not simply a coincidence, and in fact is a new viable technique. 


\subsubsection{Effect of Workpiece Gap Separation}

Results obtained from several other interesting experiments are presented in this section, performed with the newly developed AE spike/contact length calculation technique. A HSS workpiece sample was cut into two equal parts, each finish ground with $90^{\circ}$ entry and exit edge angles. Electrical discharge machining was implemented to precisely cut the hardened workpiece by sinking a thin copper electrode through the sample. The separation distance between the two workpieces could now be controlled to investigate the AE RMS response. Figure 4.19 shows a sketch of the new workpiece setup.

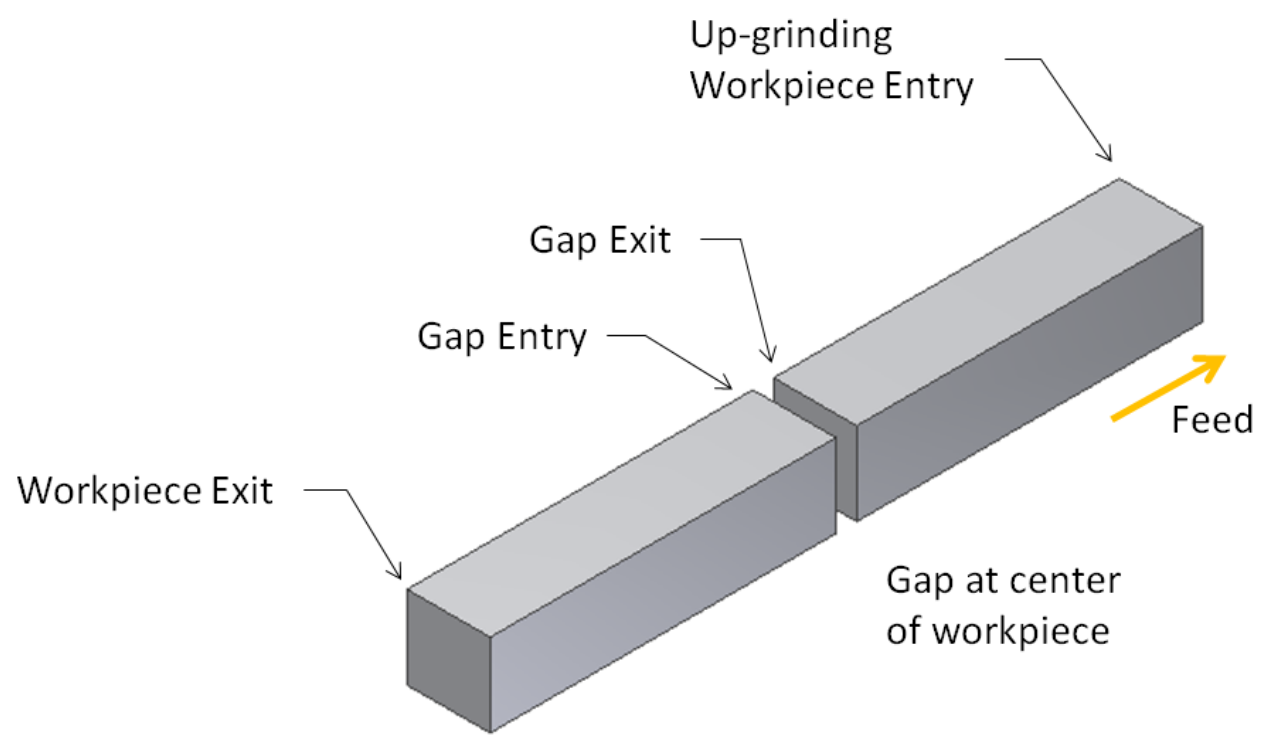

Figure 4.19: HSS workpiece with a physical gap introduced at its center.

With the previous findings in mind, a simple model for predicting the physical gap separation between two workpieces was developed. Figure 4.20 illustrates the proposed principle of AE spike existence under varying gap size conditions. 

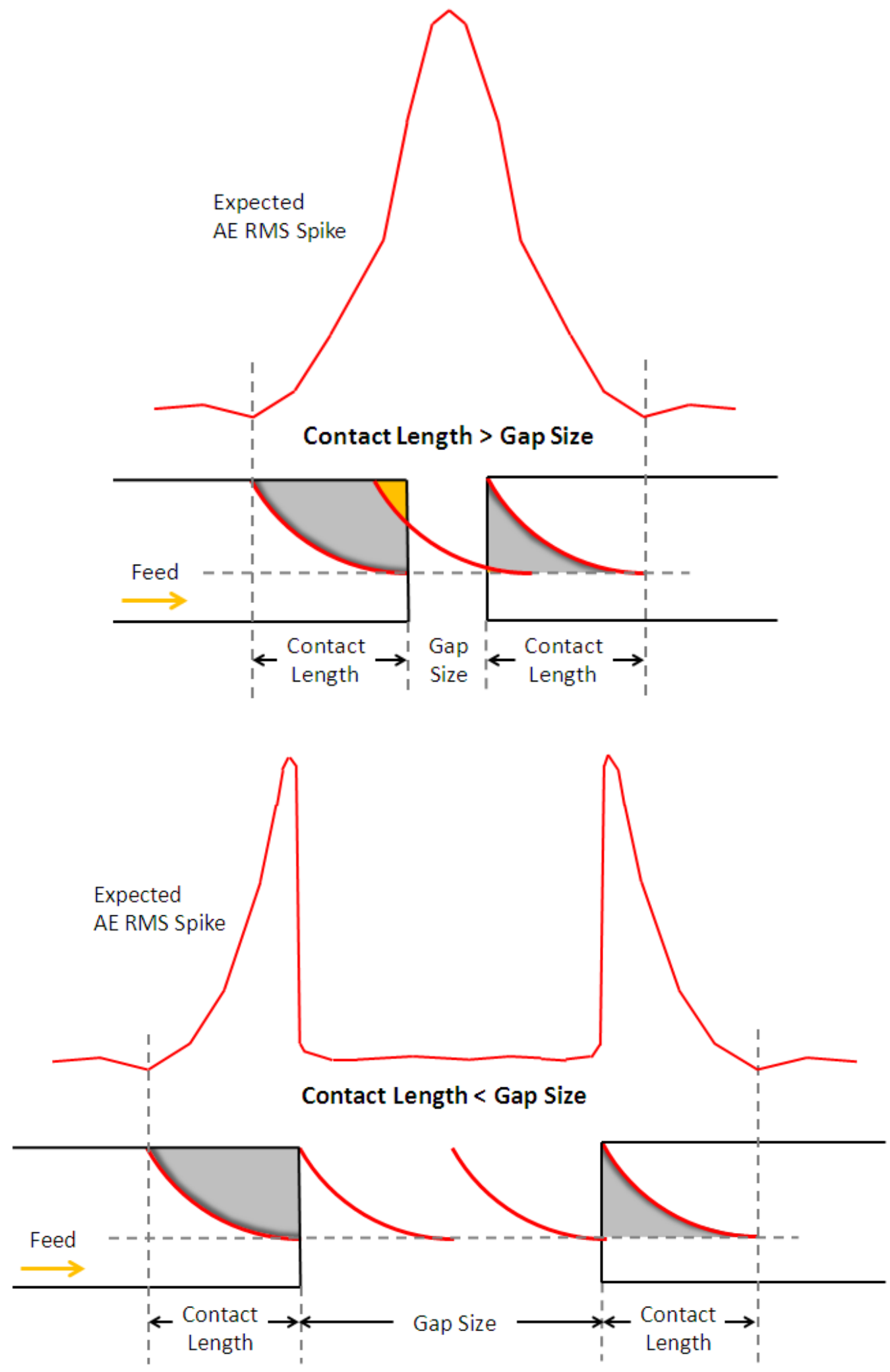

Figure 4.20: Proposed principle of AE spike behavior when gap size is less than or greater than the actual contact length. 
The duration of the complete AE spike at the gap section should follow the predicted trend, being equal to twice the actual contact length plus the specified gap size. Therefore, the estimation of the physical distance between the two workpieces can be estimated using equation 4.2.

$$
\text { Length }_{\text {gap }}=\text { AE Spike Duration } \text { gap }-2 \text { (Contact Length) }
$$

It should be noted that the spike duration values were calculated based on the beginning of the first exit spike and the end of the secondary entry spike for gap sizes greater than the actual contact length.

To test this concept, several surface grinding experiments were performed using the parameters listed in Table 3.1 and the actual contact length was calculated to be approximately $6.5 \mathrm{~mm}$. The physical gap size between the two work samples was set to values greater and less than the contact length to represent both cases of predicted spike behavior as shown in Figure 4.20. The actual AE RMS signals are shown in Figure 4.21. 


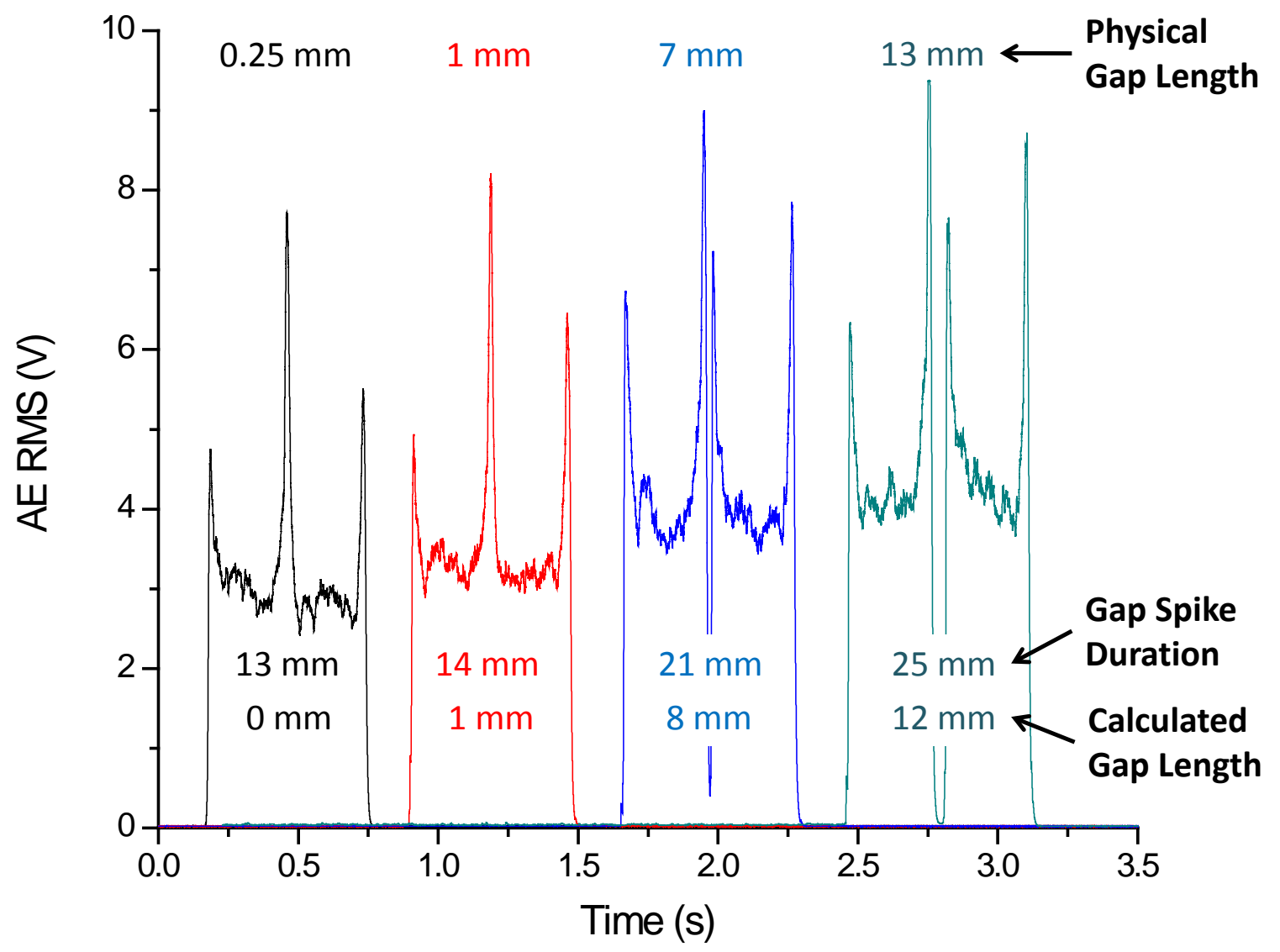

Figure 4.21: AE RMS results based on varying workpiece gap separation. The physical gap lengths and AE spike durations are displayed, along with the calculated gap length using Equation 4.2.

For separation distances of less than the actual contact length, it can be seen that the gap entry and exit spikes are combined into one, agreeing with the prediction. However, as the gap between the exit of the first piece and the entry of the second is increased to $7 \mathrm{~mm}$, two distinct AE spikes are seen. It is interesting to note that for this particular gap size, the AE RMS signal does not return to zero amplitude in between the gap like it does during the $13 \mathrm{~mm}$ gap test. This is because the $7 \mathrm{~mm}$ gap size is very similar to the actual contact length $(6.5 \mathrm{~mm})$ as calculated for this set of experiments. Therefore, this $7 \mathrm{~mm}$ separation closely represents the threshold value whereby one single AE spike separates into two distinct entry and exit spikes. 
The experimental gap lengths were calculated using equation 4.2 to see how they compared to the actual values. Although the results include some measurement error, it was found that they provide an acceptable estimation of the gap length.

Additional experiments focused on the influence of workpiece gap separation were performed, and will be discussed in a later section as the results pertain to raw AE signal analysis. 


\subsubsection{Effect of Feedrate}

As an additional way of verifying this newly proposed contact length estimation method, experiments were performed with varying grinding parameters in mind. Thus far, the workpiece feedrate was maintained constant at approximately $10.2 \mathrm{~m} / \mathrm{min}$. Literature [32,35] reports that the actual contact length increases with an increase in feedrate. This is primarily due to increased local deformation as a result of greater normal forces. Experiments were performed using $90^{\circ}$ workpiece edge geometry the grinding conditions listed in Table 3.1 while only varying the feedrate. The AE RMS results are shown in Figure 4.22.

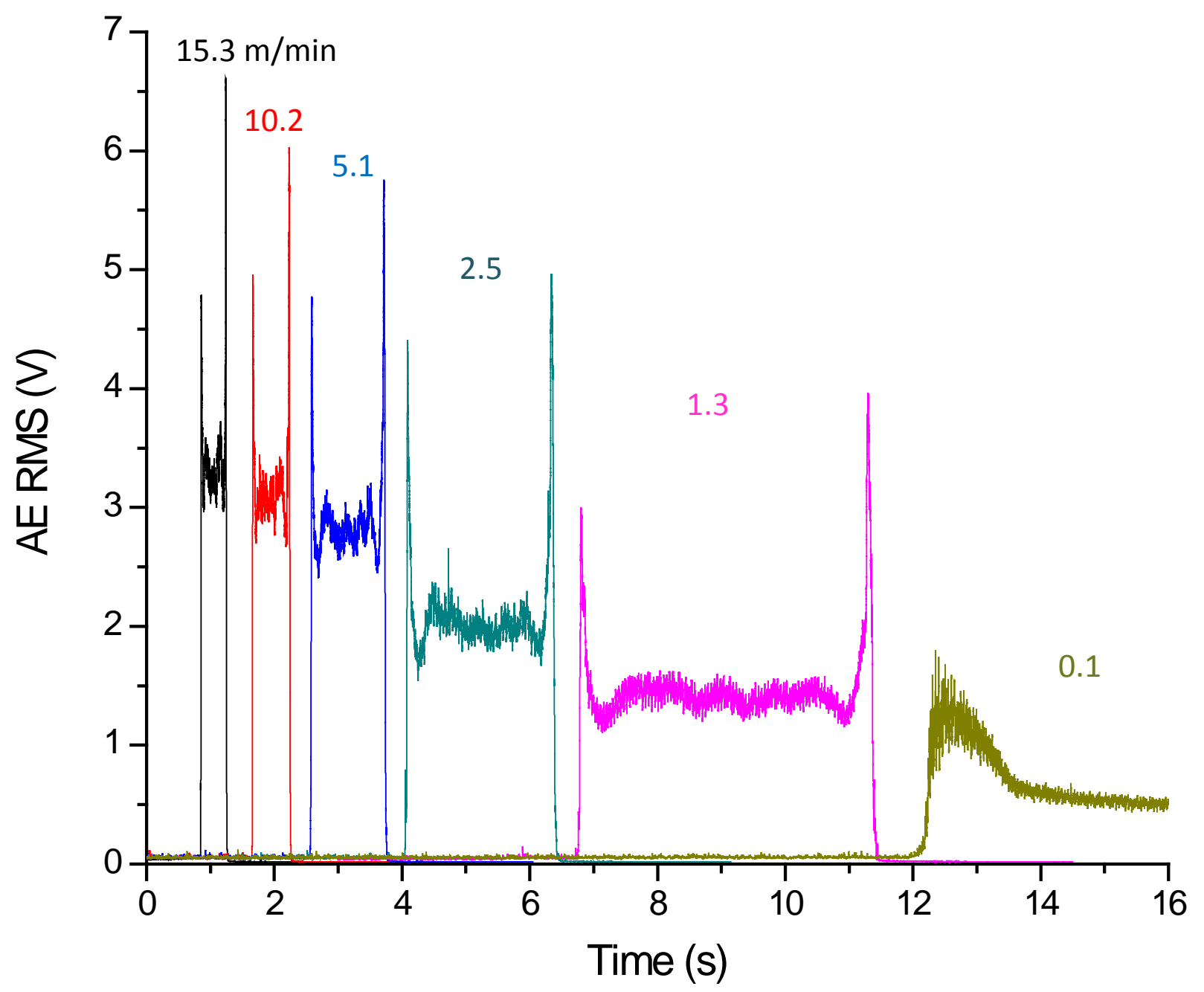

Figure 4.22: AE RMS signal comparison based on varying feedrate. 
It can be seen how the overall amplitude of the AE signal decreases with a decrease in the feedrate, but the AE entry and exit spike phenomenon remains in all cases. The respective contact lengths were calculated and plotted as a function of increasing feedrate, shown in Figure 4.23.

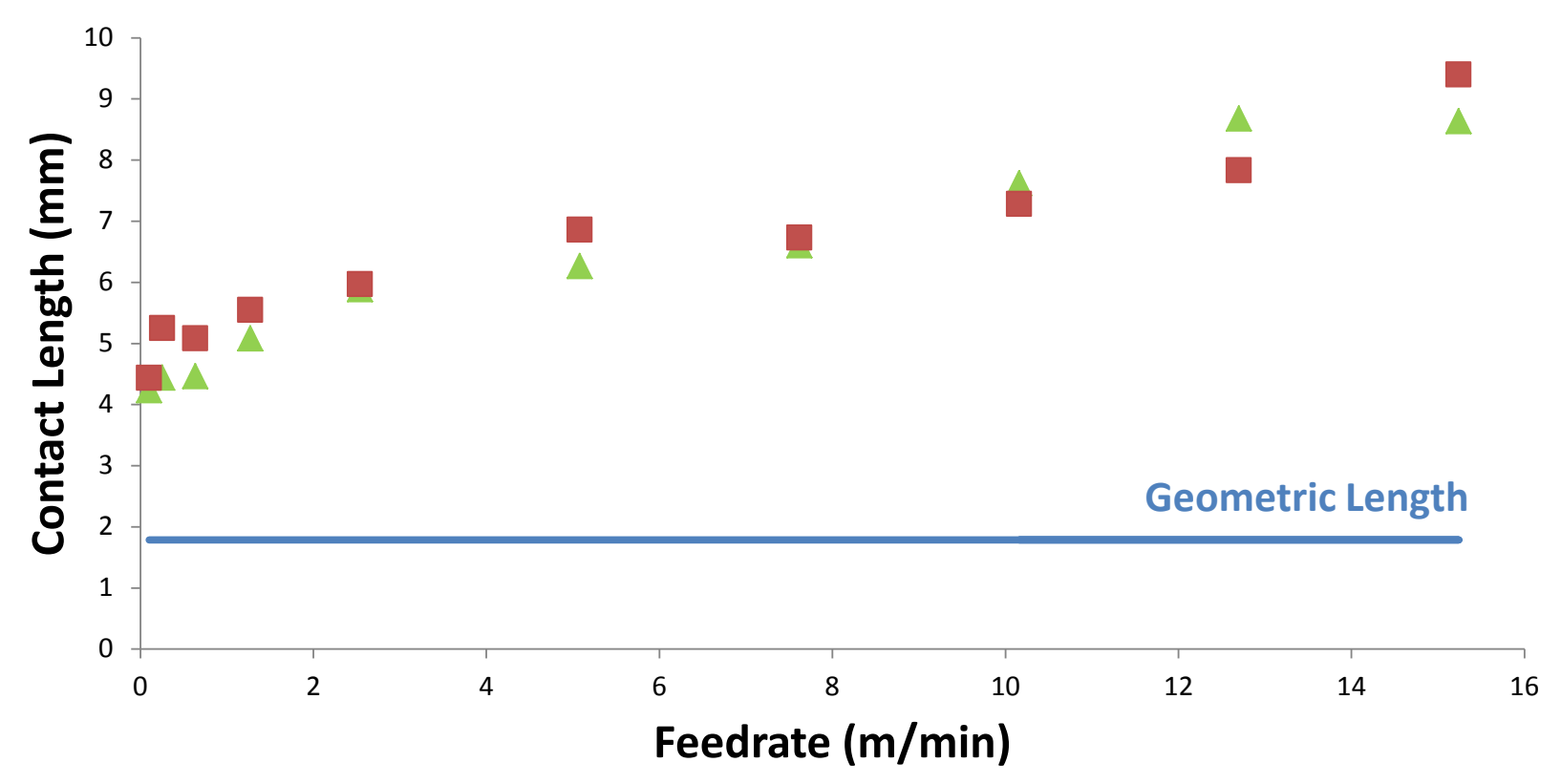

$\triangle$ Entry Spike Exit Spike

Figure 4.23: Calculation of the contact length at varying feedrates using the AE spike duration method.

This result clearly shows that the actual contact length increases with an increase in the workpiece feedrate. This result also proves that the AE entry and exit spikes are definitely not caused by a single acoustic burst event, but indeed sustained until full wheel engagement is made with the workpiece. This can be seen when looking at Figure 4.22 and the cases referring to very low feedrates. Under certain circumstances the AE spikes can be sustained for up to 2 seconds.

An interesting AE RMS signal trend is displayed as the grinding conditions are varied; higher feeds result in a 'louder' acoustic response. An investigation of the spindle speed will next be introduced to determine if this parameter also affects the RMS signal. 


\subsubsection{Effect of Grinding Wheel Speed}

Similar to the previous set of experiments involving feedrate, the effect of changing the grinding wheel speed on the AE RMS signal was investigated. Conditions listed in Table 3.1 were used, and the wheel speed was varied from approximately $5 \mathrm{~m} / \mathrm{s}$ up to $25 \mathrm{~m} / \mathrm{s}$. The results are plotted in Figure 4.24.

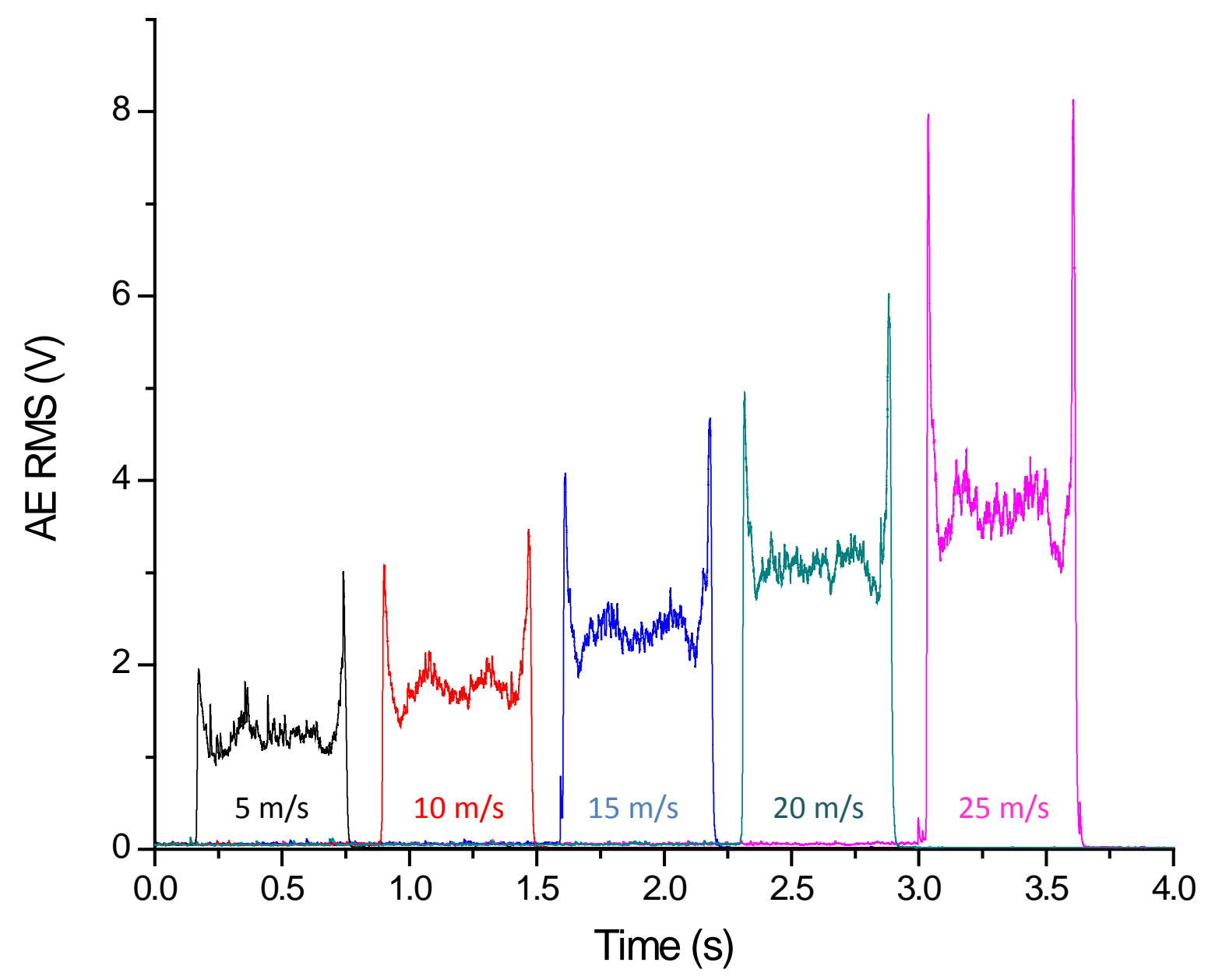

Figure 4.24: AE RMS signal comparison based on varying wheel speed.

Results show that the AE entry and exit phenomenon remains present in all cases, however, the entire signal becomes 'louder' with an increase in wheel speed. This is somewhat similar to the trend found when the workpiece feedrate was increased. Acoustic emissions are 
based on rapid local stress redistributions within a material. As the velocities at the chip interface are increased, the amplitude of the AE signal can be expected to reflect this change as a variety of thermal and mechanical effects depend on these parameters. However, it becomes difficult to separate the underlying effects that each contribute to the complete acoustic signal using the current method of RMS analysis alone.

A new approach must be taken in order to gain a deeper understanding of AE spike existence in particular, as well as identify the individual components that combine to yield the RMS signals presented thus far. The following section will hence introduce the analysis of raw acoustic emission, and demonstrate how it can help in explaining this phenomenon. 


\subsection{Raw AE Signal}

Thus far, the AE RMS spike phenomenon has been investigated in terms of its magnitude and duration in regards to a variety of grinding conditions. This has resulted in the development of a useful new technique for estimating the actual contact length between the grinding wheel and the workpiece.

Recalling the results presented in Figure 4.8, it is evident that AE RMS entry and exit spike amplitudes are dependent on the workpiece geometry. However, a physical understanding of why the AE amplitude is different at the workpiece entry and exit regions is required, keeping in mind that if not for changes in mechanisms responsible for acoustic emission, entry and exit spikes would not exist in the first place. As the primary goal of this research is to investigate the cause of AE RMS spikes, this section will hence focus on examining the raw AE signal to understand possible reasons for their existence.

\subsubsection{Features of Raw AE Signal}

The typical acoustic emission signals that have been studied thus far can be said to consist of three distinct regions: the entry spike, the continuous section, and the exit spike. During a surface grinding pass, whereby all parameters remain constant, it is of interest to comprehend the reasons for the AE signal behaving so differently at the entry and exit of the workpiece. RMS spike amplitudes have been seen to reach values of over $400 \%$ when compared to the largely stationary continuous section, but it still remains to be explained.

In order to investigate the differences and similarities between the entry, exit, and continuous regions, a new data collection procedure was implemented, involving simultaneous streaming of both the raw and RMS acoustic signals. Although raw sampling rates of $2 \mathrm{MHz}$ per channel added more complexity to the data acquisition and analysis, they are necessary when 
dealing with the high frequencies associated with acoustic emission. Using both AEwin and MATLAB, the raw acoustic emission signal could be observed post-process as a voltage versus time waveform.

All of the previously presented results were output directly from the AE Piezotron Coupler based on its internal RMS and time-constant module. This data was sampled using a common USB data acquisition card (see Section 3.3 for details) at a low rate of $20 \mathrm{kHz}$ as only the RMS values were being collected. With a more powerful DAQ card and a suitable PC, as outlined in Section 3.3, the raw AE signal was collected in a somewhat similar manner.

Once the raw AE data is stored, there are no limitations to the ways in which it can be manipulated. Root mean square trend-lines can be generated without the single value timeconstant constraint when using the analog RMS sensor output. Figure 4.25 shows a stream of raw AE data, with a digitally calculated RMS signal based on a 1.0 ms time-constant for comparison. The RMS signal can be seen to accurately represent the changes in the raw signal, most significantly at the workpiece entry and exit regions. 


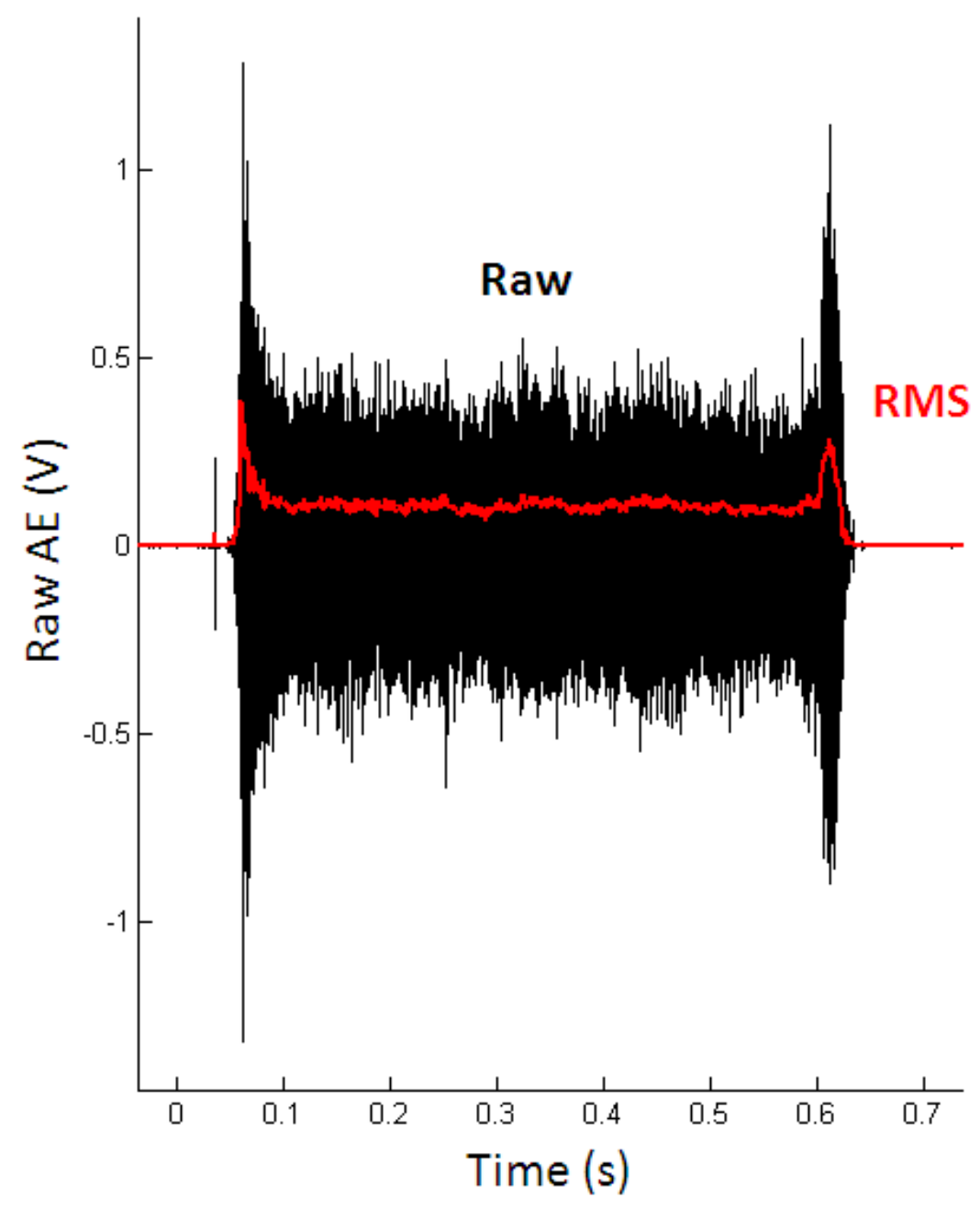

Figure 4.25: Plot of raw acoustic emission data, with a post-processed RMS signal overlaid.

It can be seen that the raw AE signal voltage alternates, whereas the RMS function averages and displays its result in the positive axis only. The raw signal below comprises over 1.4 million data points, with individual file sizes reaching several gigabytes. A detailed investigation of the raw signal has revealed that small AE bursts occur just prior to and following the much larger AE spikes at workpiece entry and exit. This is illustrated in Figure 4.26, representing the raw $\mathrm{AE}$ signal just prior to a typical workpiece entry spike. 


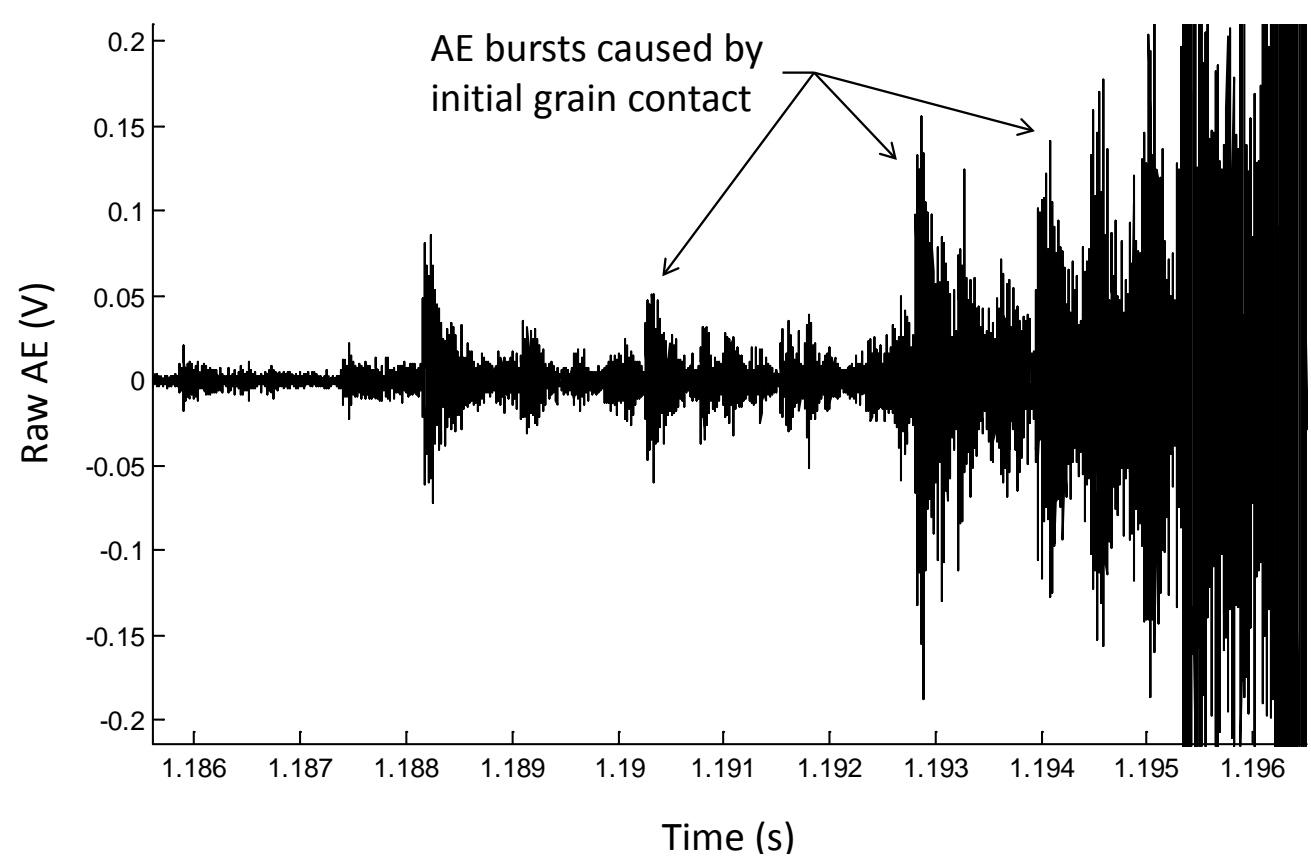

Figure 4.26: Raw AE signal representing initial grain contact during workpiece engagement.

A similar result has been reported by Webster [10], whereby higher grinding grains contact the workpiece first during the initial spark-in stage. This is also seen at the workpiece exit, and is important to the scope of this thesis. Individual random grain contact is not responsible for the AE spike phenomenon being investigated. Its effects are found to result in AE bursts which indicate instances of grain contact, but are not sustained long enough to be of significance. Figure 4.26 also demonstrates the transient nature of AE response corresponding to single grain interaction with the workpiece. Single burst durations are seen to last for only 1-2 ms in the figure above. This further verifies that the AE entry and exit spikes are not based on single AE burst events or significantly affected by the attenuation time of the signal, as their sustained duration is typically around $50 \mathrm{~ms}$.

With the raw data now available for analysis, the subsequent sections will focus on identifying the differences between the AE spike regions and the continuous signal area. An investigation of the frequency content follows. 


\subsubsection{AE Frequency Content}

Frequency analysis of the raw $\mathrm{AE}$ data allows for this complex waveform to be decomposed into consistent frequencies using the Fast Fourier Transform. Knowing the bandwidth of the $\mathrm{AE}$ sensors to be around $50-400 \mathrm{kHz}$, the frequency content of the entire $\mathrm{AE}$ signal was initially calculated to identify the dominant bands. Figure 4.27 shows a typical spectrum in perspective to the optimum sensitivity of the sensor.

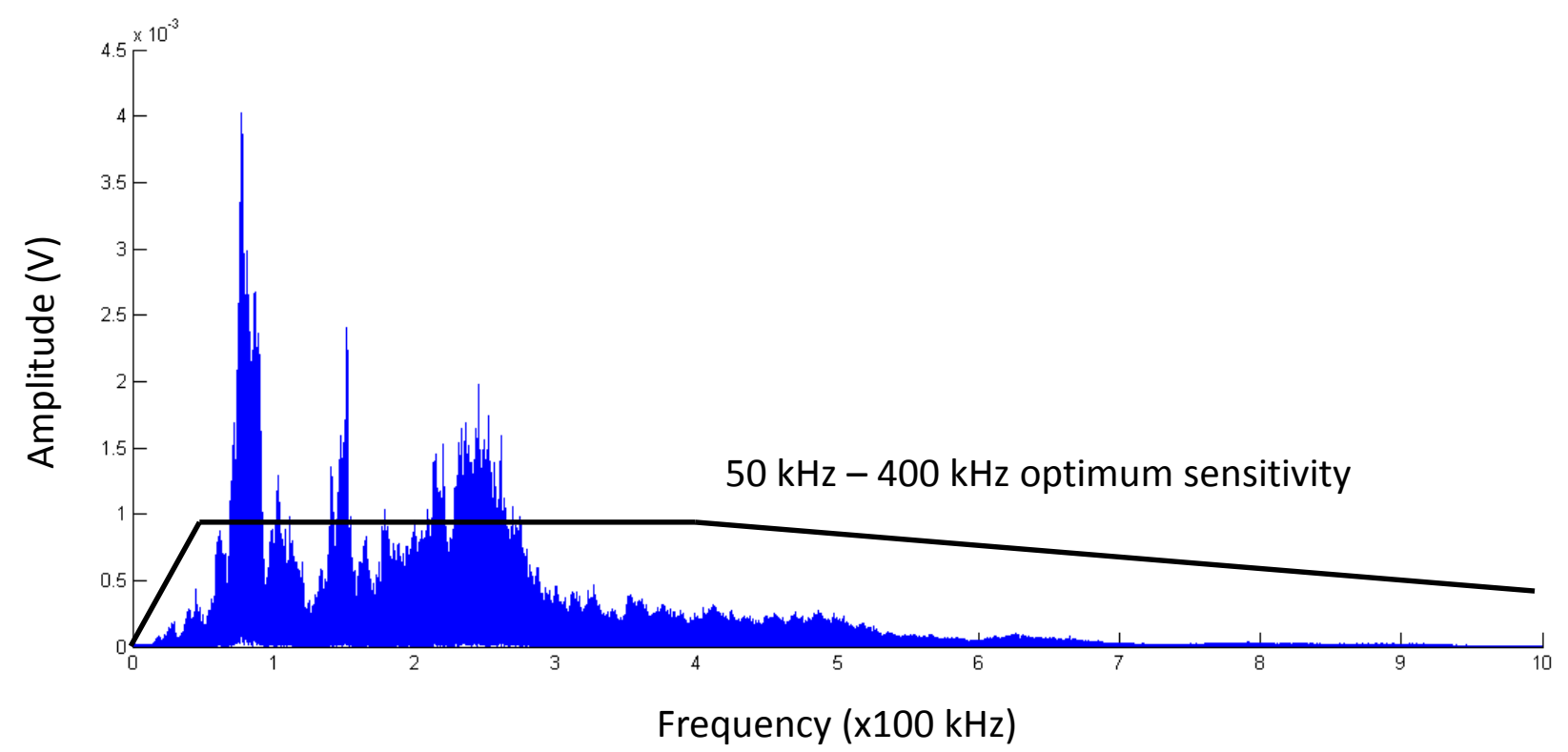

Figure 4.27: Frequency content of the entire AE signal, with reference to the response of the AE sensor.

The full frequency range up to $1 \mathrm{MHz}$ is shown here, but it can be seen that the area of our interest lies between approximately $50-300 \mathrm{kHz}$. This is well within the region of optimum sensor response, and so all future analysis will hence focus on this frequency range only. The sampling rate of $2 \mathrm{MHz}$ yields acceptable data, as it approaches 10 times the dominant frequencies seen here, avoiding any signal aliasing.

This method of analysis provides useful information regarding the entire grinding pass, but does not separate the spike regions from the continuous section of the signal. Therefore, the 
next step of isolating the entry and exit spikes into discrete sections of data allowed for differentiating them from the rest of the AE signal.

Fast Fourier Transforms were performed on individual sections of the raw AE data using MATLAB, yielding the results shown in Figure 4.28.
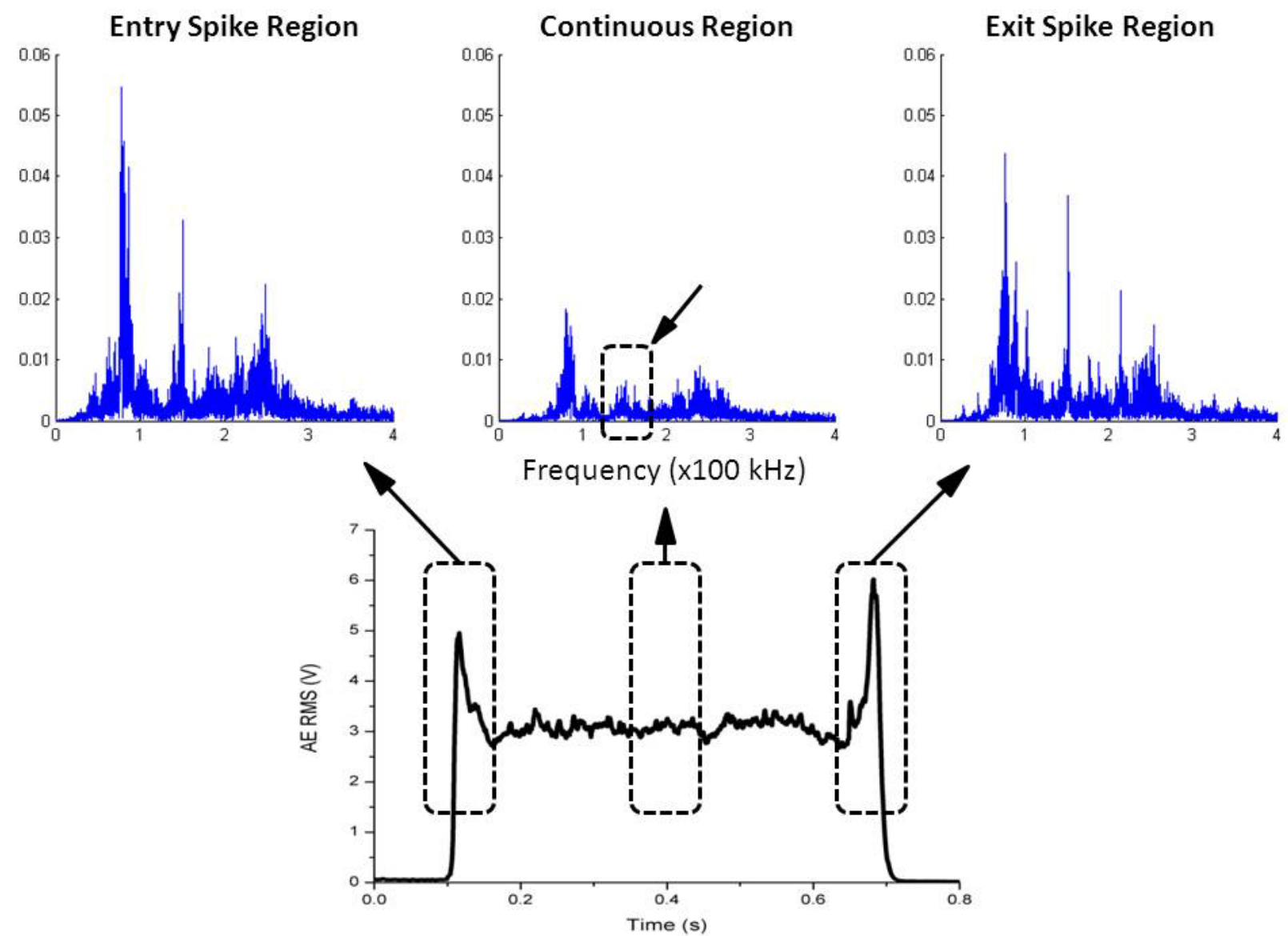

Figure 4.28: Comparison of frequency content between the entry, continuous and exit regions of the AE signal.

The frequency analysis is of particular interest when comparing the entry and exit spikes to the continuous region of the AE signal. Although not identical, the spike regions show a resemblance to one another, with a distinguishable difference to the frequency content corresponding to the middle of the workpiece. There appears to be three dominant frequency 
bands shared by the entry and exit regions around $85 \mathrm{kHz}, 150 \mathrm{kHz}$, and $250 \mathrm{kHz}$. This is of interest when compared to the frequency content of the continuous region. Of course this response is lower in overall amplitude when compared to the spike regions, but the more important aspect is the relative behavior of the three frequency bands. As highlighted in Figure 4.28, the $150 \mathrm{kHz}$ band in the continuous region is found to be much less dominant when compared to its relative amplitude in the spike regions.

The segmented AE frequency analysis method can be used to distinguish the entry and exit spike regions from the rest of the signal, but further examination is required to understand the cause of these frequency differences. A literature search pertaining to similar AE frequency patterns shown in Figure 4.28 led to preliminary findings associated with grinding burn. Figure 4.29 is from the work of Liu et al. [23] and depicts the AE signals resulting from two different grinding experiments: the first with grinding burn, and the second without. The general frequency pattern is of interest here, as similar trends exist in the current work as well.

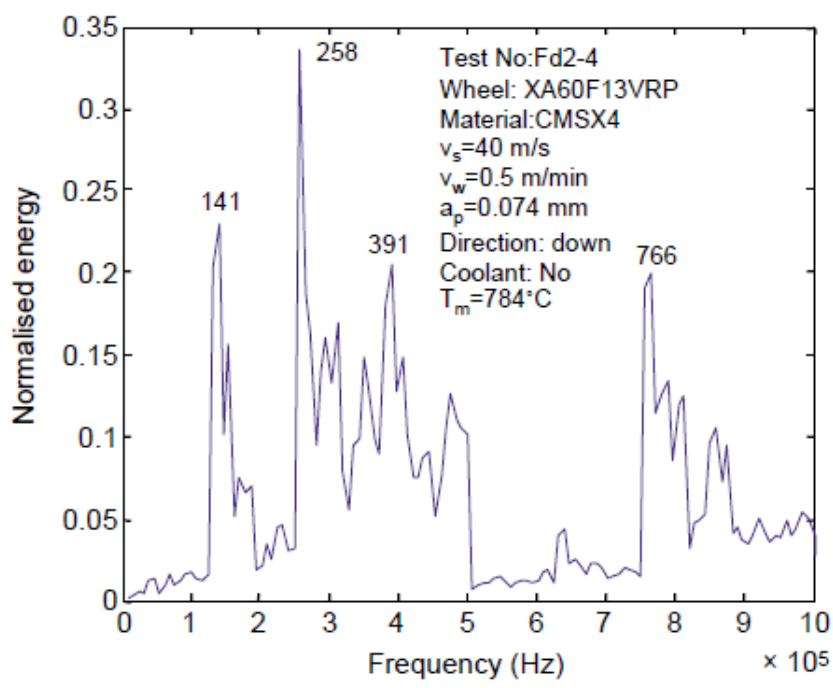

(a) With grinding burn

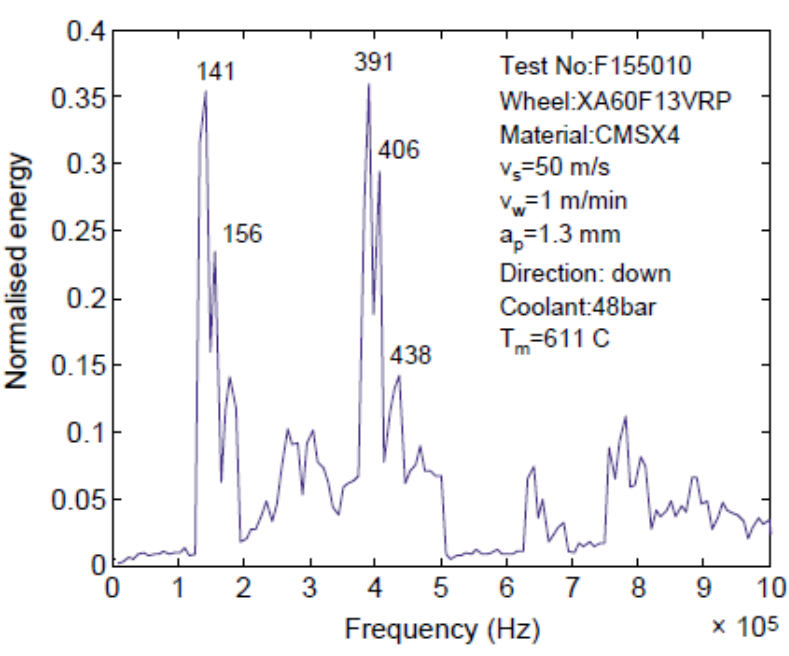

(b) Without grinding burn

Figure 4.29: Frequency comparison of grinding scenarios a) with burn and b) without burn [23]. 
Although the corresponding frequencies are greater than in this thesis, a striking trend is still seen in the lower end of the spectrum. For the situation without grinding burn (Figure 4.29b), the AE frequency plot shows two dominant spikes around 141 and $391 \mathrm{kHz}$, without any notable activity in between these bands. When considering the results linked to grinding burn (Figure 4.29a), a significant frequency band around $258 \mathrm{kHz}$ is excited that falls between the two spikes in question. The actual frequency values in [23] are found to be shifted to higher values compared to our work by approximately 50-100 kHz. Considering that Liu et al. [23] used dissimilar data analysis methods, as well as performed experiments using a completely different setup, this discrepancy is noted but is deemed acceptable. The manifestation of grinding burn in the frequency content of the AE signal is discussed in the following section.

\subsubsection{Grinding Burn and AE Frequency Content}

Examination of the AE frequency content has provided the basis for a new hypothesis that grinding burn is responsible for the difference in the frequency content between the spike and continuous $\mathrm{AE}$ signal sections. In order to determine if grinding burn is indeed occurring at the workpiece entry and exit regions, a similar data comparison was made in consideration of Liu's work [23].

Referring to the previous set of results presented in Figure 4.10 of Section 4.1.5, grinding burn was observed to occur during the contact length experiments. At depths of cut greater than $30 \mu \mathrm{m}$, the surface of the workpiece became darker in colour with a visibly lower surface finish than those observed after passes with a depth of cut of $10 \mu \mathrm{m}$. It was visually evident that grinding burn was occurring under these conditions, and so the raw AE data corresponding to these experiments was analyzed and compared to the previous frequency results in Figure 4.28. 

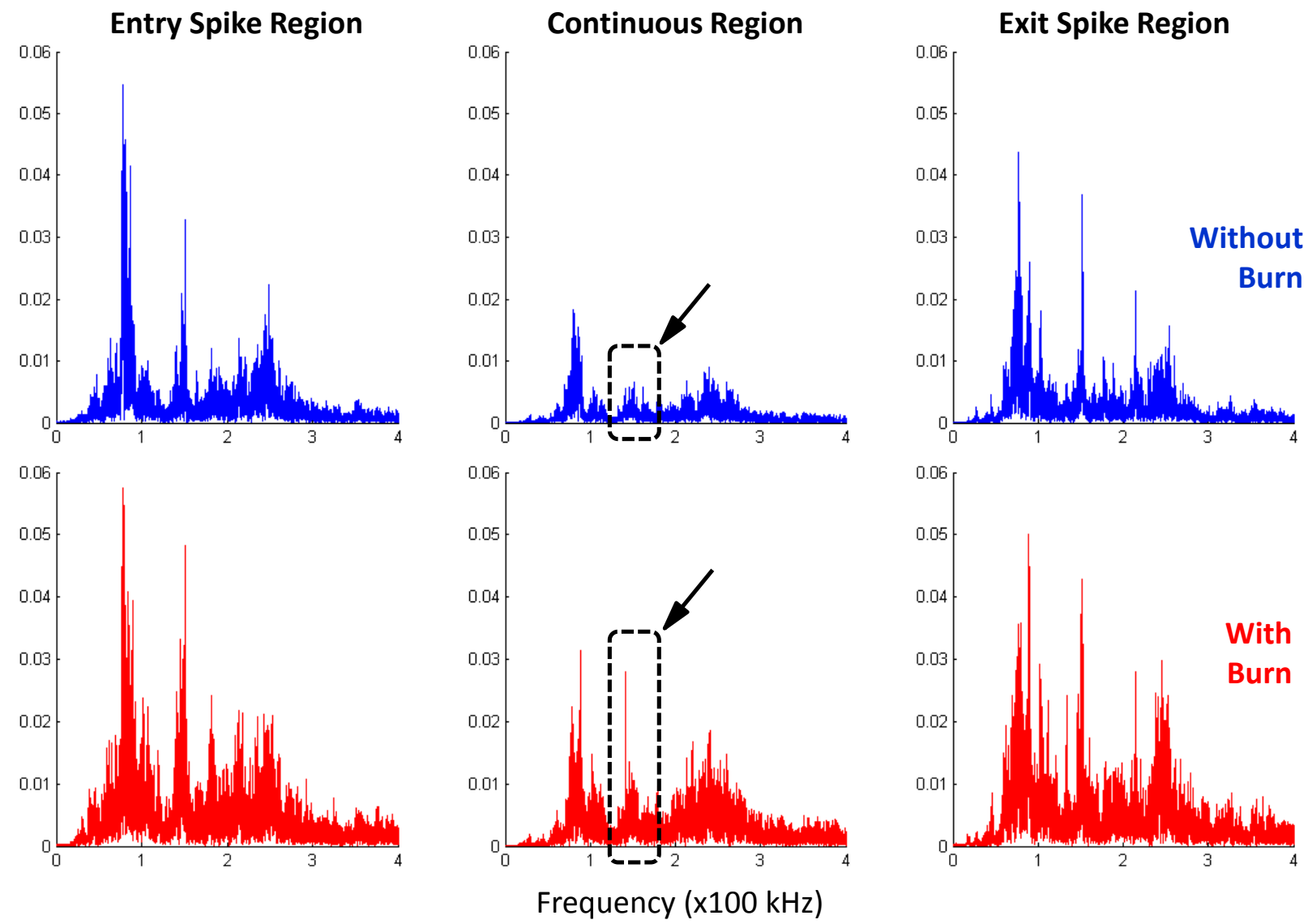

Figure 4.30: Frequency content comparison of discrete AE signal regions. The top result corresponds to the case of no grinding burn at a $10 \mu \mathrm{m}$ depth of cut, while the bottom signifies grinding burn at a $40 \mu \mathrm{m}$ depth of cut.

Figure 4.30 compares the frequencies of the new grinding burn data to the previously presented frequency results in each of the three AE signal regions. When comparing the spike regions, the burned workpiece frequencies are seen to be very similar to the sample without burn. However, in the continuous region, the previously "missing” $150 \mathrm{kHz}$ frequency band is shown to become significantly more dominant in the continuous region of the burned workpiece, as highlighted in Figure 4.30. The frequency content behavior of this new continuous region now remarkably resembles that of the spike regions. A better way to clearly compare this result of workpiece burn on the $150 \mathrm{kHz}$ frequency band is presented in Figure 4.31. 

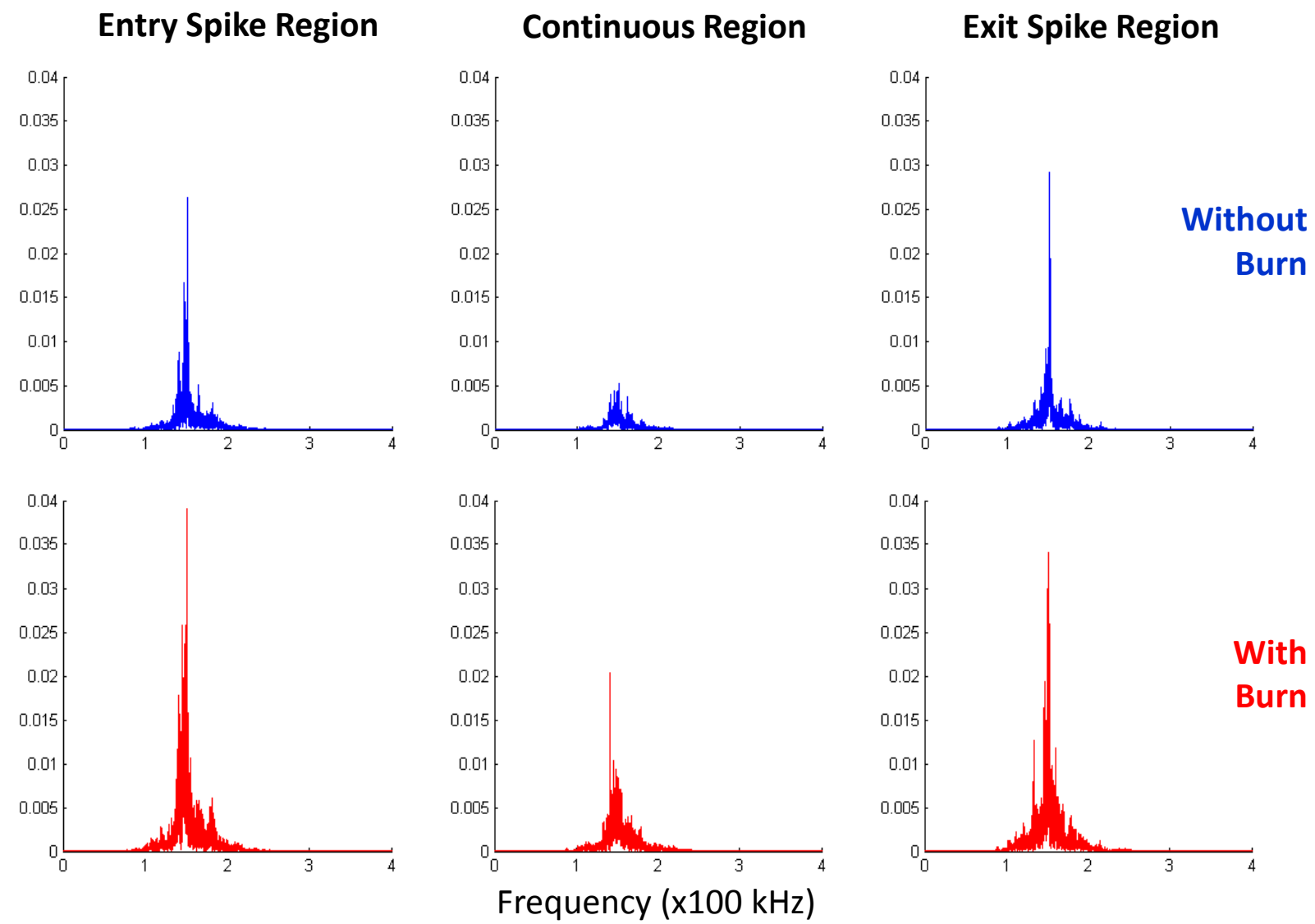

Figure 4.31: Frequency response comparison based on a 140-160 kHz filtered signal.

The same data is presented here, but a $140-160 \mathrm{kHz}$ band-pass filter was first used to eliminate all other frequencies in the raw AE waveforms. This figure clearly shows the significant effect that grinding burn has on this particular frequency band. Increases in frequency components over $100 \mathrm{kHz}$ have been reported to relate to the generation and progression of grinding burn in the literature [26], but more interestingly, the experimental frequency content also agrees with Liu’s findings [23] in Figure 4.29. Therefore, the frequency analysis supports the hypothesis that grinding burn is occurring at the workpiece entry and exit regions, contributing impart to AE spike existence. 
An interesting effect was soon noticed during the analysis of experimental results, pertaining specifically to the $150 \mathrm{kHz}$ frequency band and coolant penetration into the grinding zone. When manually selecting the discrete AE data segment representing the continuous signal region, an area located near the center of the workpiece was typically chosen, as highlighted in Figure 4.28. Grinding conditions resulting in no workpiece burn have been shown to minimize the $150 \mathrm{kHz}$ frequency band in this region. However, when the same continuous signal segment was observed much closer to the entry spike region, the amplitude of the $150 \mathrm{kHz}$ frequency was noticeably higher.

An investigation of this occurrence eventually led to the conclusion that the coolant was not effectively penetrating into the grinding zone during the first instance of workpiece engagement. This is due to the fluid being directed into the side of the workpiece rather than into the grinding zone as desired. In some grinding applications, the use of a sacrificial workpiece therefore becomes necessary to effectively channel the coolant into the grinding zone. Figure 4.32 illustrates the implementation of a sacrificial workpiece and how it provides better coolant penetration at the workpiece entry region. 


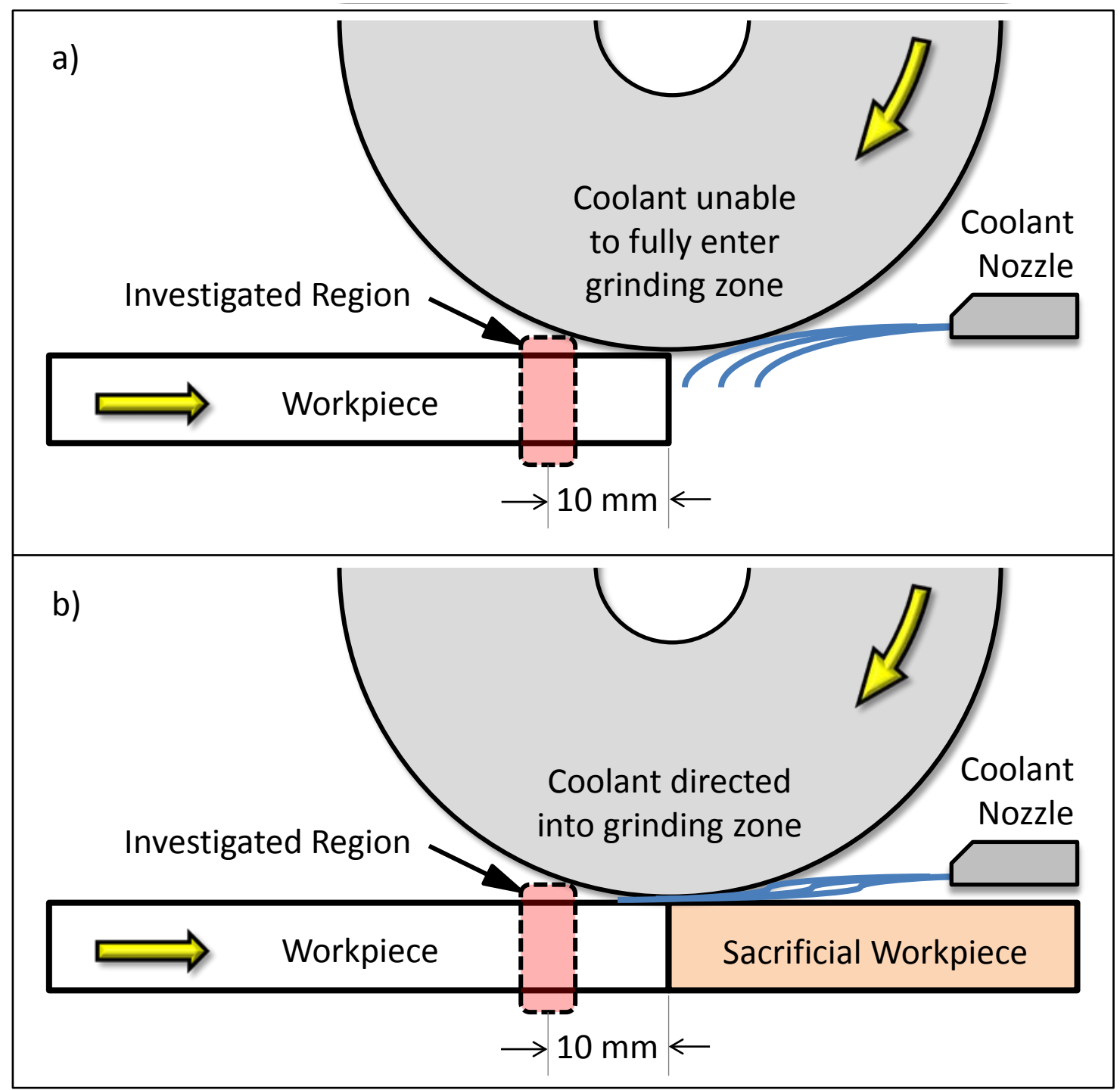

Figure 4.32: Illustration of workpiece entry region to demonstrate the effect of coolant penetration into the grinding zone in scenarios a) without a sacrificial workpiece and b) with a sacrificial workpiece.

In the case of up-grinding, illustrated in Figure 4.32, the level of coolant penetration into the grinding zone at wheel entry significantly increases when the sacrificial workpiece is used. As a consequence of the coolant nozzle location and the feedrate direction, a similar effect occurs at the workpiece exit region during down-grinding. A segment of each workpiece is highlighted in the figure which corresponds to the respective region of the continuous AE signal for investigation. The frequency content of these sections was compared, demonstrating the 
effect of the sacrificial workpiece on the $150 \mathrm{kHz}$ band. Figure 4.33 presents the filtered results of the 'burn' frequency at the same workpiece location for each of the two scenarios, with reference to its contribution within the entry spike region.

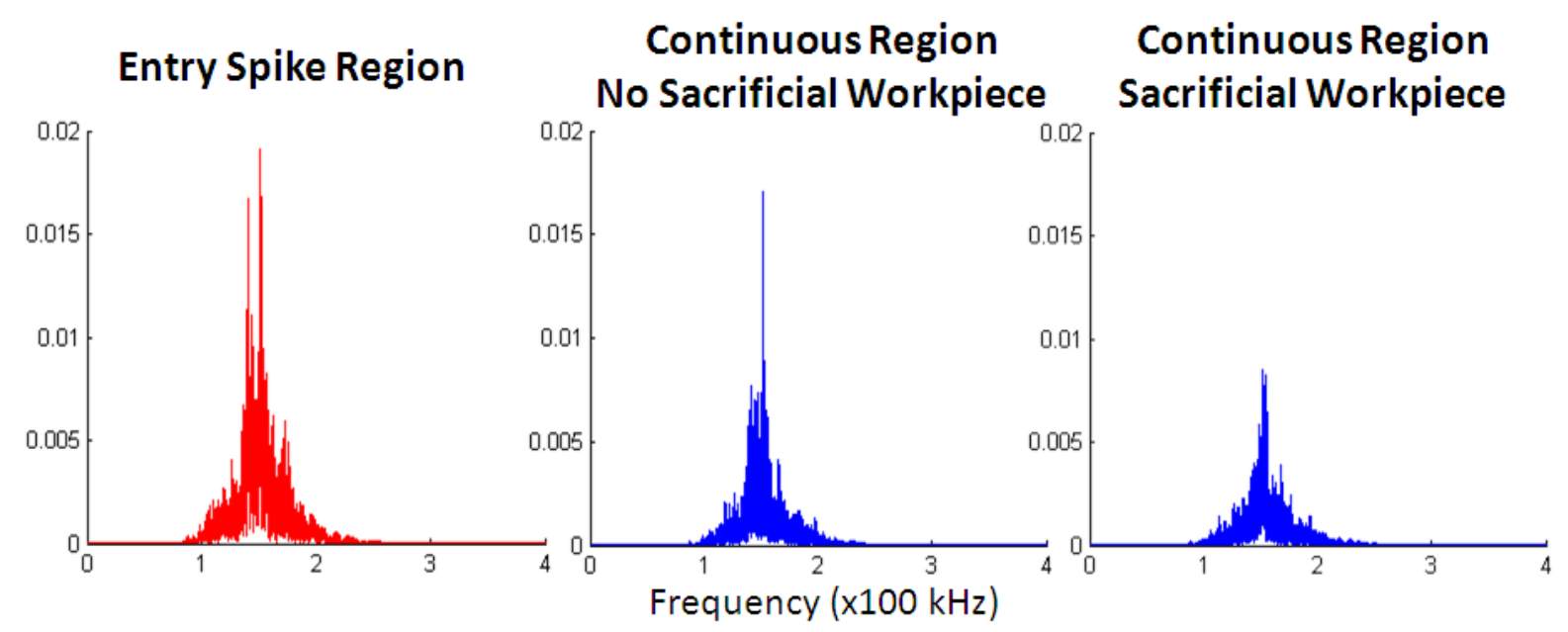

Figure 4.33: Filtered frequency results demonstrating the effect of a sacrificial workpiece on the $140-160 \mathrm{kHz}$ frequency range. Continuous AE sections $10 \mathrm{~mm}$ from entry edge are compared to the entry spike region.

The outcome shows the effect of adding a sacrificial workpiece on the $150 \mathrm{kHz}$ frequency band. At a location relatively close to the AE entry spike, the amplitude of the 'burn' frequency in the continuous region decreases as a result of adding a sacrificial workpiece. This is due to the increased coolant penetration into the grinding zone, lowering the workpiece temperature. As the location from the engagement edge is increased, the influence of the sacrificial workpiece becomes less relevant as the workpiece itself channels the fluid into the grinding zone.

The possibility of using this result for on-line monitoring of grinding burn is significant. The designation of grinding burn is currently somewhat of a gray area, with most methods based purely on visual workpiece inspection $[\mathbf{2 6 , 3 6}$. Classifying the degree of grinding burn, or identifying the instance when it first occurs can be crucial in the manufacture of function-critical, 
high value components. Simply using surface appearance as an indication of grinding burn can be misleading, as a simple spark-out pass can be performed to remove the visually observed 'burned' surface. The underlying thermal effects that affect workpiece integrity may still remain and go undetected which can be a significant problem. This observation is supported by Malkin [24], as he reported that "temper colours are usually removed by spark-out at the end of the grinding cycle...but this effect is cosmetic and the absence of temper colours on the ground surface does not necessarily mean that workpiece burn did not occur.” Recent research $[\mathbf{2 3}, \mathbf{2 6}, \mathbf{2 7}]$ has hence focused on the real-time detection of grinding burn, particularly using acoustic emission sensors. Results have shown promise, but still remain either process-specific or slightly convoluted.

The concept of 'unseen' grinding burn is of particular interest when referring to Figure 4.10 for example. The five individual grinding passes of varying depth of cut were performed in random order, even though the grinding wheel was dressed and the workpiece was initially pre-ground to ensure uniform contact before each pass. The random effect can be seen in the results when considering the actual experimental order of: $20,10,40,30,50 \mu \mathrm{m}$ depths of cut respectively. The visual signs of grinding burn were observed at $30 \mu \mathrm{m}$ and higher, but when referring to the experimental order, the $40 \mu \mathrm{m}$ pass corresponded to the first onset of thermal damage within the workpiece.

It can be seen in Figure 4.10 that the $30 \mu \mathrm{m}$ pass is actually greater in amplitude than the $40 \mu \mathrm{m}$ pass, as perhaps the underlying effects remained from the previous test resulting in workpiece burn. As mentioned before, even though several spark-out passes were performed to 'remove' the burn in between tests, this result proves that the effects of grinding burn may indeed remain just under the workpiece surface. Grinding burn is a definite contributor to the 
acoustic emission signals, but its effect is still not understood in terms of AE entry and exit spike existence, which is the primary focus of this work.

The following section will involve a discussion of why grinding burn may be occurring at workpiece entry and exit regions, with an effort to describe its effect on the AE spikes phenomenon.

\subsubsection{Why does Grinding Burn occur at Entry/Exit Region?}

Previous AE RMS results have shown the significant influence of workpiece entry and exit geometry on spike existence. Referring to the recently developed hypothesis which states that grinding burn occurs at the workpiece entry/exit region, a new investigation will be made regarding a possible relationship between grinding burn and workpiece geometry.

Reports on transient thermal effects during surface grinding state that temperatures can exceed the steady-state value once workpiece material becomes suddenly unavailable to conduct the heat away $[\mathbf{2 4 , 2 9 ]}$. This principle was used to explain the premise of grinding burn occurring at workpiece entry and exit, as the edge geometry significantly influences the amount of bulk workpiece material surrounding the contact length transition region. As the entry/exit angle decreases, more local workpiece material becomes available to act as a heat sink, conducting the heat away from the grinding zone. Figure 4.34 compares the instant of grinding wheel engagement between three cases of workpiece geometry. For the same depths of cut, higher temperatures are expected at the entry/exit region of the $135^{\circ}$ workpiece as there is less bulk material available. 


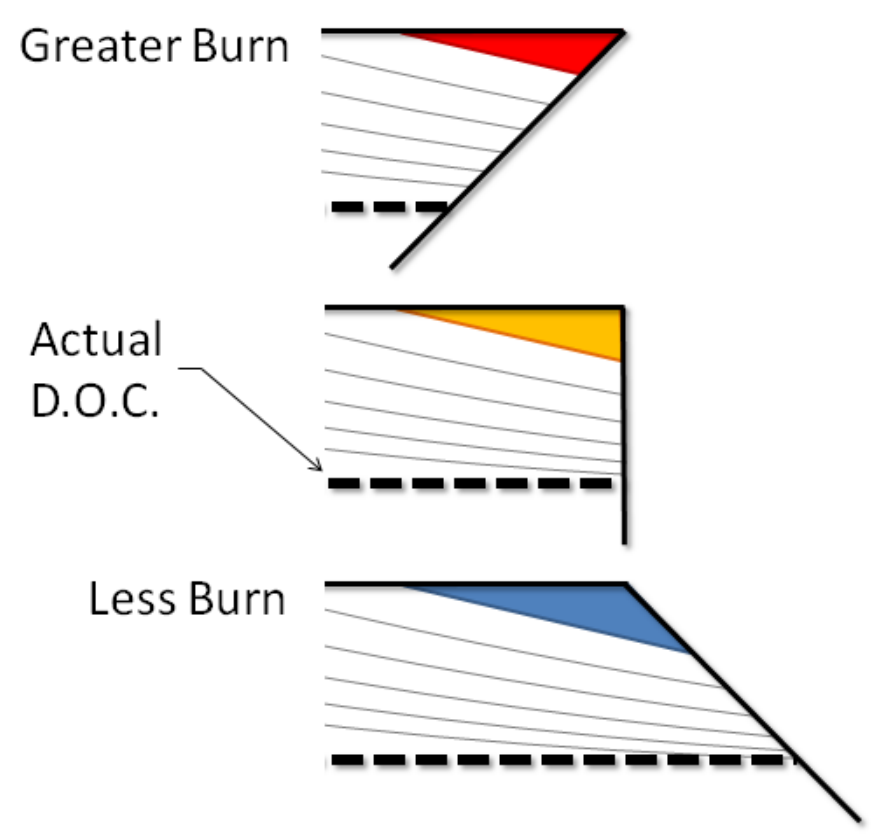

Figure 4.34: Illustration of how workpiece edge geometry affects the amount of bulk material surrounding the entry/exit regions. Varying levels of grinding burn result within the contact length transition zone.

It must be noted that this figure describes the thermal behavior within the contact length transition zone, and not at the actual finished workpiece surface. Even though the final surface may not show any effects of grinding burn at the corresponding AE entry/exit spike regions, it is because these 'burned' regions of the workpiece have been removed as chips. Once the full contact length becomes established, the amount of bulk workpiece material reaches the maximum value and thereafter remains constant, resulting in a decrease of the grinding burn frequency band, as seen in Figure 4.28.

A similar theory regarding elevated temperatures within grinding burr regions has been reported also by Aurich et al [21]. This basis can be used to explain the previously reported burr effects on AE RMS spike behavior in Section 4.1. Increased AE spike amplitudes were found at workpiece edges corresponding to more significant burr formation. Therefore, a similar argument can be made here regarding thermal effects on the AE signal. Aurich states that 
"during the short-time grinding of the small burr volume, the temperature in the burr strongly increases” [21]. He continues to explain that this comes as a direct result of reduced heat conduction due to the lack of surrounding workpiece material.

It has been found that $\mathrm{AE}$ entry and exit spike amplitudes are influenced by both workpiece geometry and local grinding burn within the entry/exit regions. Raw acoustic analysis has shown the contribution of individual frequency bands at discrete sections of the AE signal. The primary focus has thus far been on the $150 \mathrm{kHz}$ 'burn' frequency band, but the remaining $85 \mathrm{kHz}$ and $250 \mathrm{kHz}$ bands deserve investigation.

In order to compare the complete contribution of each of these frequency bands, they have been displayed in terms of their RMS values. The original raw signal was filtered within the frequency range of interest and the root mean square of each individual data set was calculated. The results for the $85 \mathrm{kHz}$ and $150 \mathrm{kHz}$ bands are first investigated in Figure 4.35, comparing their responses between varying workpiece geometries. 


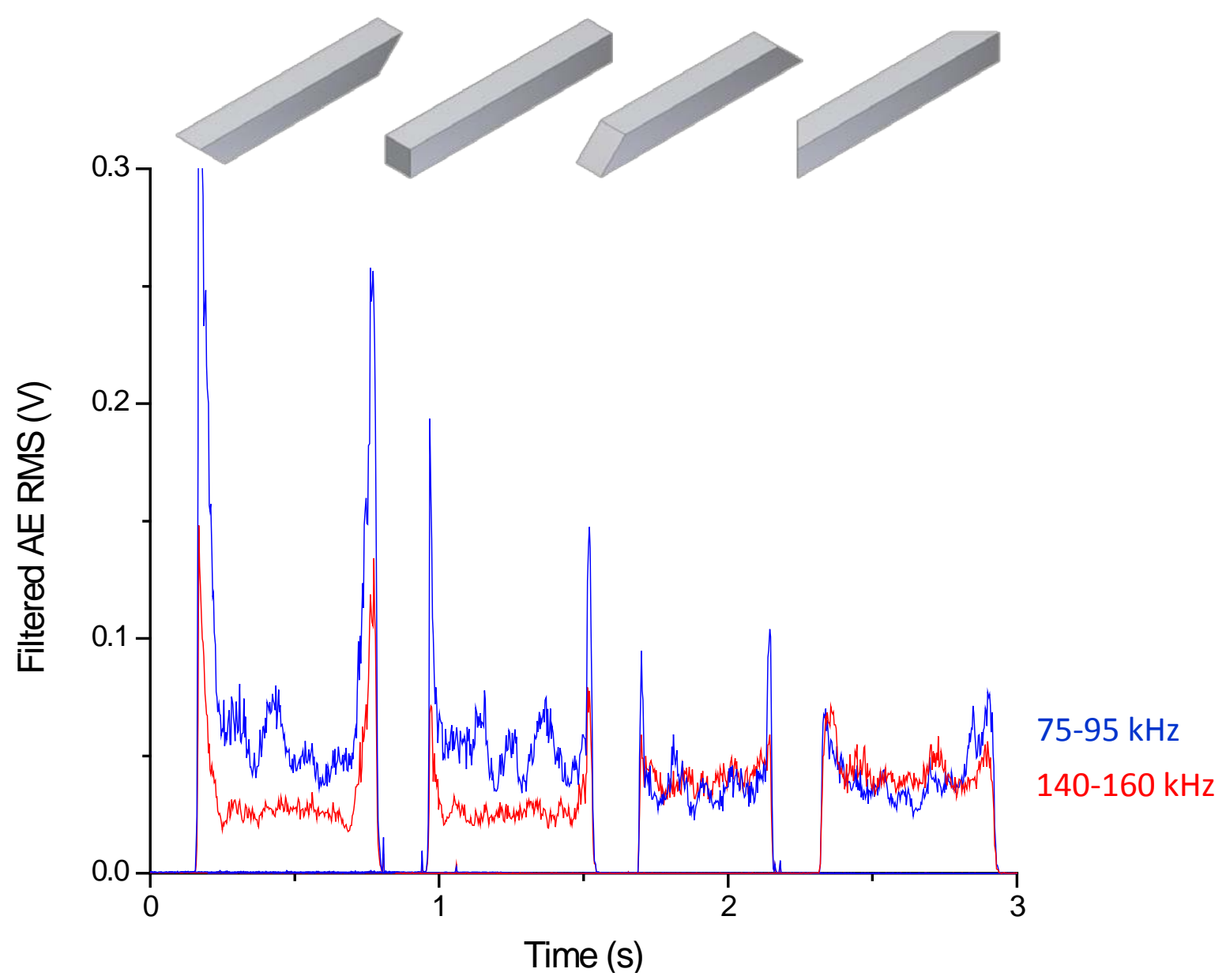

Figure 4.35: Filtered AE RMS frequency component comparison with varying workpiece entry/exit geometry.

The results show the individual response of the two frequency bands for varying workpiece geometry. It also suggests that these two frequency bands are independent, as they can be seen to respond uniquely. This can be seen in the spike regions when considering the result corresponding to the $45^{\circ}$ workpiece geometry. In this case, entry/exit spikes are found to be insignificant in terms of the 'burn' frequency band (140-160 kHz component), but are evident in the $75-95 \mathrm{kHz}$ component. This provides reason to believe that there are more than just thermal effects responsible for the AE spike phenomenon.

The fourth data set is of particular interest, corresponding to an entry/exit region with gradual width. This geometrical configuration was shown to yield an unfiltered RMS signal with 
no entry/exit spikes whatsoever (Figure 4.8), and is the only configuration showing a significant minimization of the $75-95 \mathrm{kHz}$ component. This finding suggests that the physical impact or dynamic response of the grinding wheel and the workpiece at the entry/exit region may be associated with this frequency component. However, it is difficult to prove this concept based on this set of data alone as the contribution of both frequency components are seen to change between each case. The next section will therefore discuss how this issue was addressed and present the results associated with a new experiment. 


\subsubsection{Distinguishing the Origin of AE Frequency Components}

It has been shown that changing the workpiece entry/exit geometry does not provide enough information to distinguish the effects of individual frequency components, creating the need for a new experiment. Focus was hence placed on controlling only one of these components at a time using the recently acquired knowledge regarding grinding burn that the $150 \mathrm{kHz}$ burn frequency responds to elevated workpiece temperatures at the entry/exit region, controlled by the amount of heat conduction provided by surrounding bulk material.

Creating a scenario by which the level of entry/exit burn could be controlled without changing the workpiece geometry would provide the necessary conditions for frequency analysis. This was achieved by introducing two different workpiece gap geometries as illustrated in Figure 4.36.

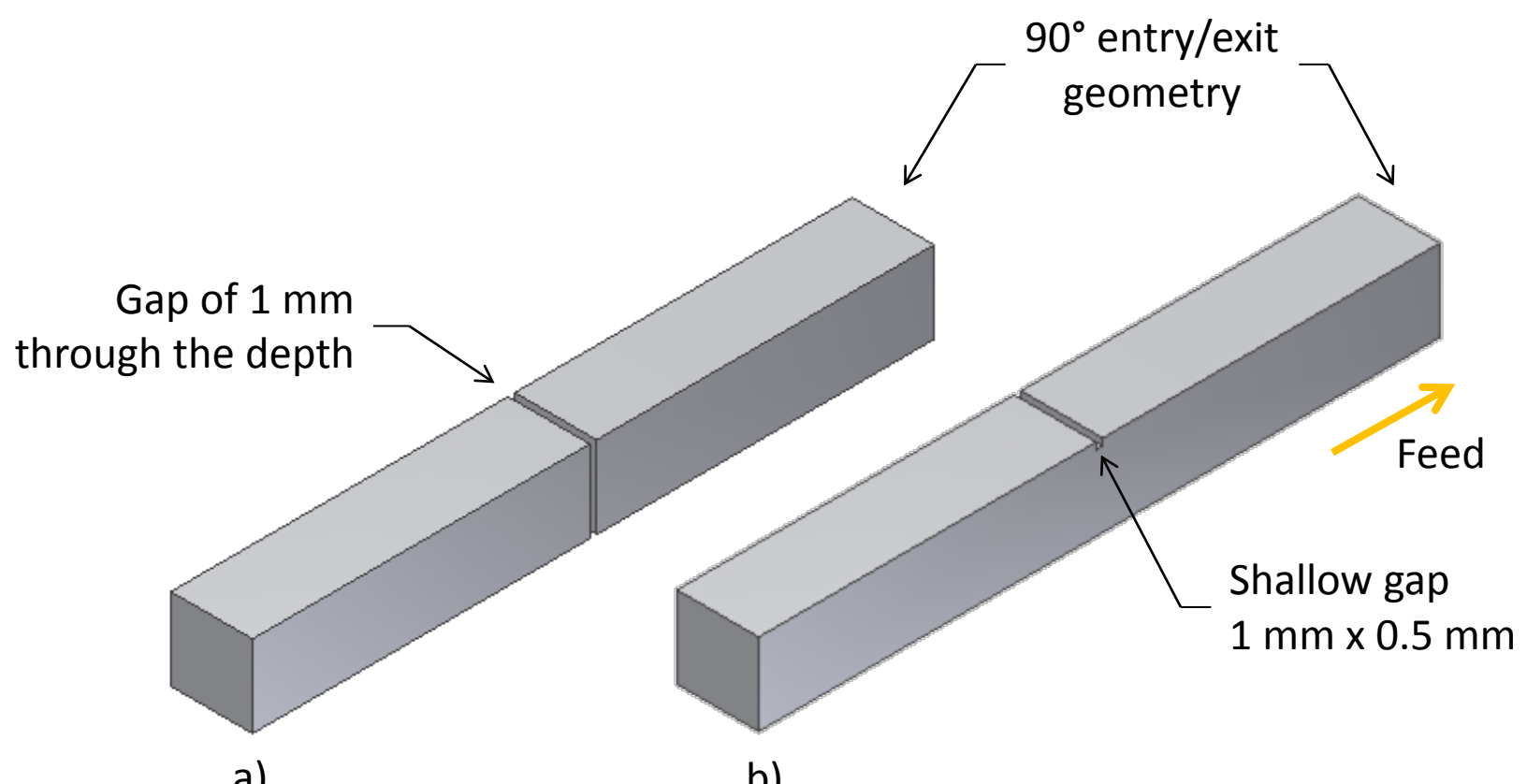

Figure 4.36: Illustration of different gap scenarios. Workpiece a) has a completely through-gap, while b) has a gap depth of only $0.5 \mathrm{~mm}$. Gap width was kept constant in both scenarios at $1 \mathrm{~mm}$. 
When comparing the physical gap regions of the two workpieces, the geometry of the entry/exit regions remains the same, but the amount of bulk material surrounding this area is different. Based on previous results, the burn frequency should be expected to be higher at the full gap region than at the shallow gap as there is less bulk material available to act as a heat sink. It should be noted that the gap width of $1 \mathrm{~mm}$ is less than the actual contact length of the grinding wheel, and so a single $\mathrm{AE}$ spike will correspond to this region as discussed in Section 4.1.5.2. The filtered AE RMS results comparing just the gap regions are shown in Figure 4.37.

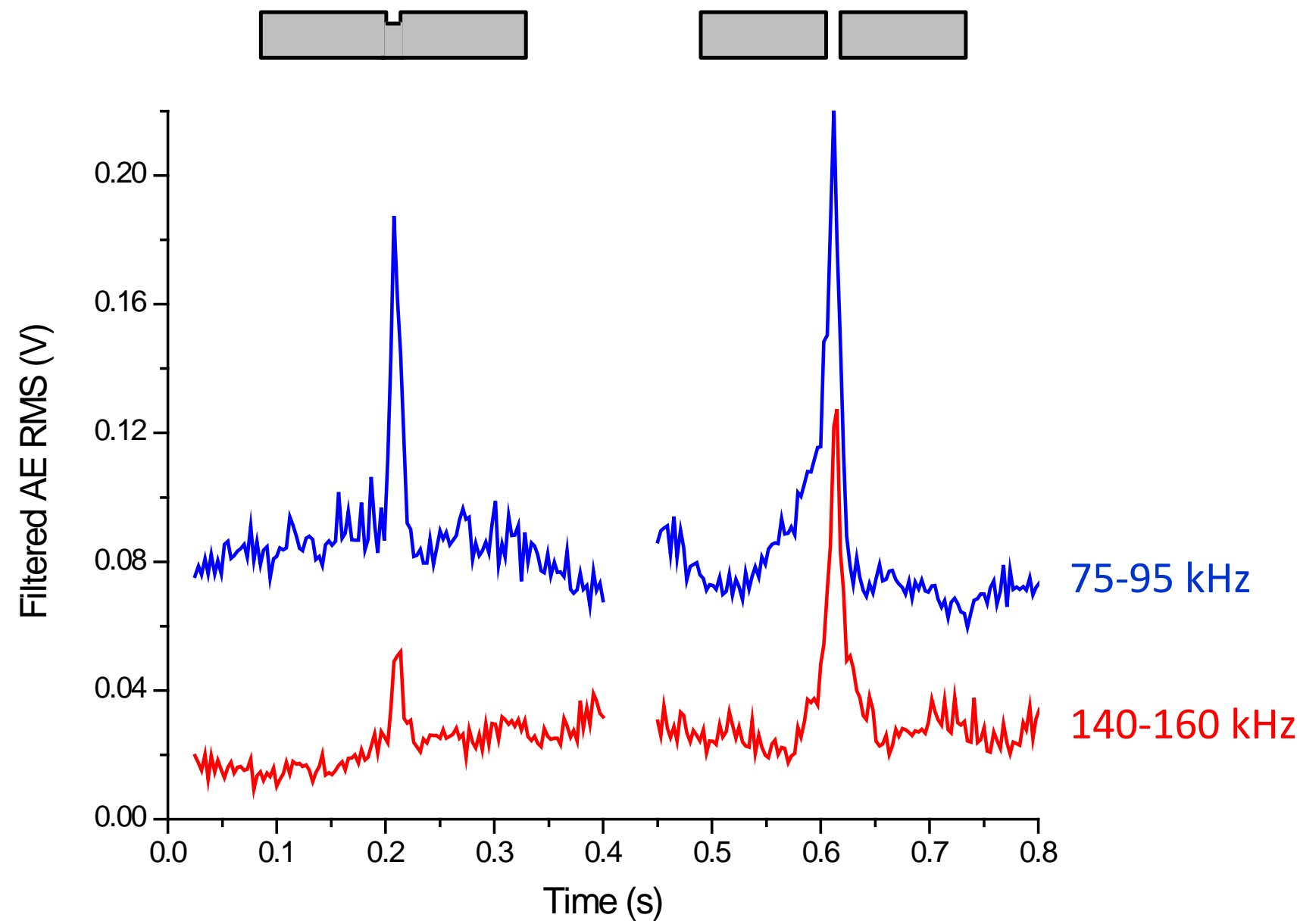

Figure 4.37: AE RMS filtered frequency components at the gap-region only, separated for clarity. 
The 75-95 kHz band can be seen to contribute very similarly to spike amplitude in each gap scenario, whereas the burn frequency component shows a significant difference. This result confirms the premise that the first and second frequency bands behave independently and that their influence on spike existence is caused by distinct phenomena. Although the geometric interaction of wheel and workpiece is the same in each case, the overall amplitude of the AE spike at the gap region can vary significantly as a consequence of thermal effects.

This result suggests that the 75-95 kHz frequency band is not influenced by temperature, but instead is dependent on the physical interaction between the grinding wheel and workpiece. As previously found in Figure 4.35, the AE spike contribution of this component increases with more aggressive entry/exit geometries. This is seemingly related to the level of damping available in the grinding zone as the contact length becomes fully developed.

Inasaki et al. [39] have reported that "grinding damping increases in proportion to the length of the contact between the wheel and the workpiece," and that an increase of the grinding damping has the general effect of making the entire system more stable. Matsumoto et al. [7] have found that acoustic emissions are associated with a similar level of stability during their work on chatter detection. It is reported that "AE events can be observed as the contact becomes unstable”. These findings agree with the patterns found in the $75-95 \mathrm{kHz}$ frequency component.

Damping of the grinding wheel reaches a maximum once full wheel contact is made, corresponding to the continuous AE signal region, but the system behaves differently at workpiece entry and exit. In these regions the system is less damped than when compared to complete wheel engagement, which explains the corresponding AE behavior.

Furthermore, the result also defends the manner in which the burn frequency is influenced and how it contributes to entry/exit spikes. Just as the AE spike amplitude was seen 
to increase with an increase in entry/exit geometry in Figure 4.35, it also increases as a result of lesser bulk material surrounding identical entry/exit geometries. This further verifies that the $150 \mathrm{kHz}$ component is temperature dependent and not simply reliant on workpiece geometry, but instead the thermal consequences it induces.

Thus far, the so-called ‘burn’ frequency has been designated as only the $150 \mathrm{kHz}$ band, but grinding burn has been reported to affect acoustic emission frequencies generally greater than $100 \mathrm{kHz}$ [26]. Therefore, the influence of the third significant frequency component of 240-260 kHz must be investigated to see if also relates to thermal effects. An identical RMS analysis of this frequency range resulted in similar behavior when compared to the original burn frequency component (140-160 kHz) as illustrated in Figure 4.38.

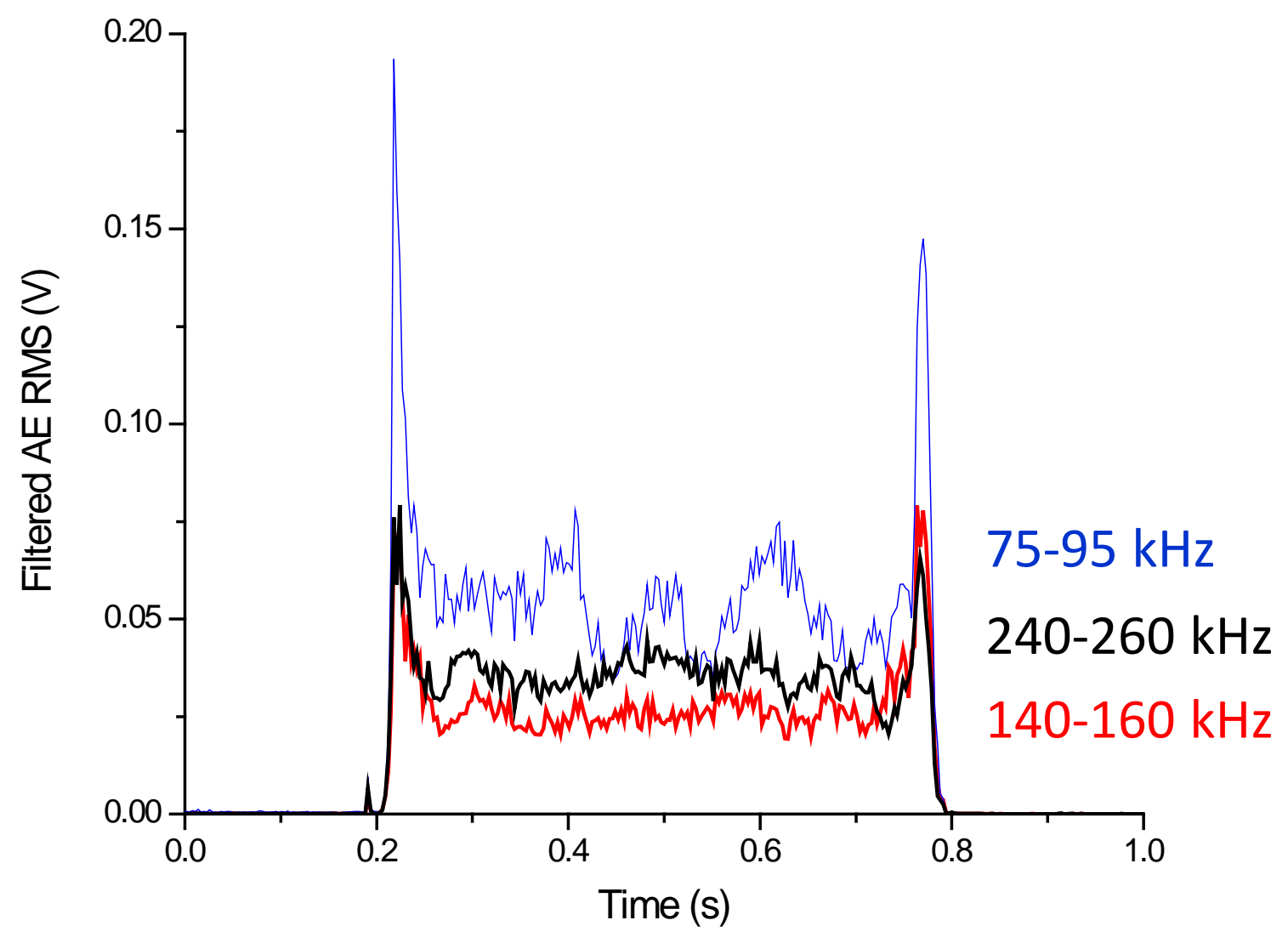

Figure 4.38: AE RMS comparison of the three most dominant frequency components. 
This result was taken from the same $90^{\circ}$ workpiece data presented previously in Figure 4.35, and clearly demonstrates how closely these two different frequency bands respond to each other when compared to the $75-95 \mathrm{kHz}$ component. Not only do the AE spike contributions behave almost identically in this example, but they were also found to respond similarly in both the varying geometry and gap experiments. When observing the continuous regions of these filtered signals, there is an overwhelmingly stronger relationship between the two frequency components greater than $100 \mathrm{kHz}$ when contrasted to the lower frequency component. This agrees with Eda's work [26] which states that grinding burn is generally associated with $\mathrm{AE}$ frequencies greater than $100 \mathrm{kHz}$, and can therefore be used to state that the third frequency band is also temperature dependent.

Although there are no single frequency components in the $240-260 \mathrm{kHz}$ band that fluctuate in amplitude as substantially as the $150 \mathrm{kHz}$ component in the $140-160 \mathrm{kHz}$ band, it seems as though a broader range of frequencies increase with temperature in this case. This frequency range may not be as ideal for monitoring grinding burn, but its overall contribution to the AE signal, most notably the entry/exit spikes, is almost equivalent to the $140-160 \mathrm{kHz}$ range.

The analysis method of decomposing the raw AE signal into its three most significant frequency components has allowed for individual acoustic events to be distinguished. The comparison of each component, as presented in Figure 4.38, identifies relative contributions to the $\mathrm{AE}$ entry/exit spike phenomenon, providing an understanding of its causes. This figure is particularly helpful in illustrating the relative contribution of each component, with the 75-95 kHz band seen to have the greatest influence. However, if the frequency components above $100 \mathrm{kHz}$ are generally considered to act as one, representing thermal effects, then their significance becomes just as influential regarding entry/exit spike behavior. 
The investigation of both RMS and raw acoustic signals has provided unique results pertaining to the AE entry and exit spike phenomenon. RMS analysis has identified the parameters that effect signal behavior and demonstrated the relationship between $\mathrm{AE}$ entry/exit spikes and workpiece geometry. However, the development of a novel technique for measuring the actual contact length between the grinding wheel and the workpiece has proven to be the most practically significant achievement of the RMS analysis.

Raw signal analysis has illustrated the cause and effect of individual frequency components that contribute to the AE spikes, with thermal effects and the level of damping within the contact length transition zones being primarily responsible. A possible technique for detecting grinding burn has also been introduced. 


\section{Conclusions and Future Work}

Acoustic emission entry and exit spikes have been investigated in order to understand their cause and to determine if they can be used to provide useful information about the surface grinding process. This chapter will summarize the results pertaining to RMS and raw signal analysis, and propose ideas for future work.

\subsection{Conclusions}

Embarking on a curiosity-driven project, the motivation for this work came solely from investigating a currently unexplained phenomenon of $\mathrm{AE}$ entry/exit spikes. This research was focused entirely on determining the reasons associated with this rather unique signal response. The results from this research are summarized as follows:

1. Burr formation is dependent on the workpiece edge geometry and does affect the AE spike amplitude in a minor way. Exit burrs are more dominant during up-grinding and entry burrs are more dominant during down-grinding and directly relate to AE spike behavior. Although grinding burrs and $\mathrm{AE}$ entry and exit spikes are weakly correlated, burr formation generally corresponds to a slight increase in spike amplitude, apparently due to thermal effects and increased plastic deformation.

2. The workpiece geometry dictates the duration time of the AE entry/exit spikes, as it defines the transition distance between the time of initial and full wheel contact. The $90^{\circ}$ entry/exit geometry has been shown to share a common point between these two instances, being ideal for a new contact length estimation technique. The AE RMS spike durations for this 
workpiece geometry can be utilized to estimate the actual contact length between the grinding wheel and the workpiece. This novel measurement technique is non-destructive, rapid, and can be performed in-situ on actual work components, unlike current techniques. The actual contact lengths range between approximately 2-3 times the corresponding geometric values as reported in the literature, mainly due to elastic deformations in the grinding zone.

Increased workpiece feedrates result in larger contact lengths due to an increase in normal force, and consequently greater deformation in the grinding zone.

3. Workpiece edge geometry has a significant effect on entry and exit spike behavior. As the entry and exit angles of the workpiece are increased, the amount of surrounding bulk material in these regions decreases. This results in an increase of local workpiece temperature due to less heat being conducted away from the grinding zone. The elevated acoustic levels in these regions are of direct consequence, as thermal loads contribute to the $\mathrm{AE}$ signal.

Furthermore, the interaction between the grinding wheel and the workpiece at the edge becomes less stable with an increase in the edge angle. This effect compounds with the thermal influence, yielding the most significant AE entry and exit spikes at increased workpiece angles.

4. Acoustic emission signals during surface grinding are primarily influenced by dynamic system behavior and thermal effects, each corresponding to unique frequency bands. The 75-95 kHz frequency band reflects the physical dynamic interaction between the grinding wheel and the workpiece, with a dependence on the damping in the system. The 
$140-160 \mathrm{kHz}$ and $240-260 \mathrm{kHz}$ ranges correspond to thermal effects, with grinding burn most notably identified by the $150 \mathrm{kHz}$ frequency component for the workpiece and setup used in this work.

5. Grinding burn is said to occur within the entry/exit regions of the workpiece, dependent on workpiece geometry. It is most distinguishable by the level of the $150 \mathrm{kHz}$ frequency band being the main differentiable feature between the $\mathrm{AE}$ spike and continuous signal region. Using AE data to identify grinding burn is advantageous in monitoring applications as it is accomplished in-process.

The detailed investigation of the AE entry and exit phenomenon has yielded significant results that can be used to better understand the surface grinding process. Research has proven that the AE signal behavior is not merely a random occurrence or caused by some obscure event such as "increased gain at the corners of the workpiece" [42]. Instead, it provides remarkably useful information about the process, including grinding burn identification and estimation of the actual contact length between the grinding wheel and the workpiece. 


\subsection{Future Work}

This section proposes suggestions on how to improve the setup used in this work as well as introduce ideas for future related investigations.

\subsubsection{Other Sensors}

Although acoustic emission analysis has been reported to be the most practical for grinding process monitoring, acceleration and force data was also investigated for comparative analysis. However, due to the physical nature of these sensors, frequency response can differ by two orders of magnitude when compared to AE data. For example, the accelerometer being used had a frequency range of $1 \mathrm{~Hz}-10 \mathrm{kHz}$, whereas the $\mathrm{AE}$ sensors respond to frequencies greater than $50 \mathrm{kHz}$.

To compare the signals of the different sensors, a single grinding pass using parameters listed in Table 3.1 was performed on a $90^{\circ}$ workpiece. The entire setup was mounted on a threeaxis dynamometer, and the accelerometer was positioned parallel to the grinding direction as shown in Figure 3.5. For further comparsion to the AE RMS results, the RMS of the acceleration data was calculated and superimposed over the raw signal shown in Figure 5.1. 

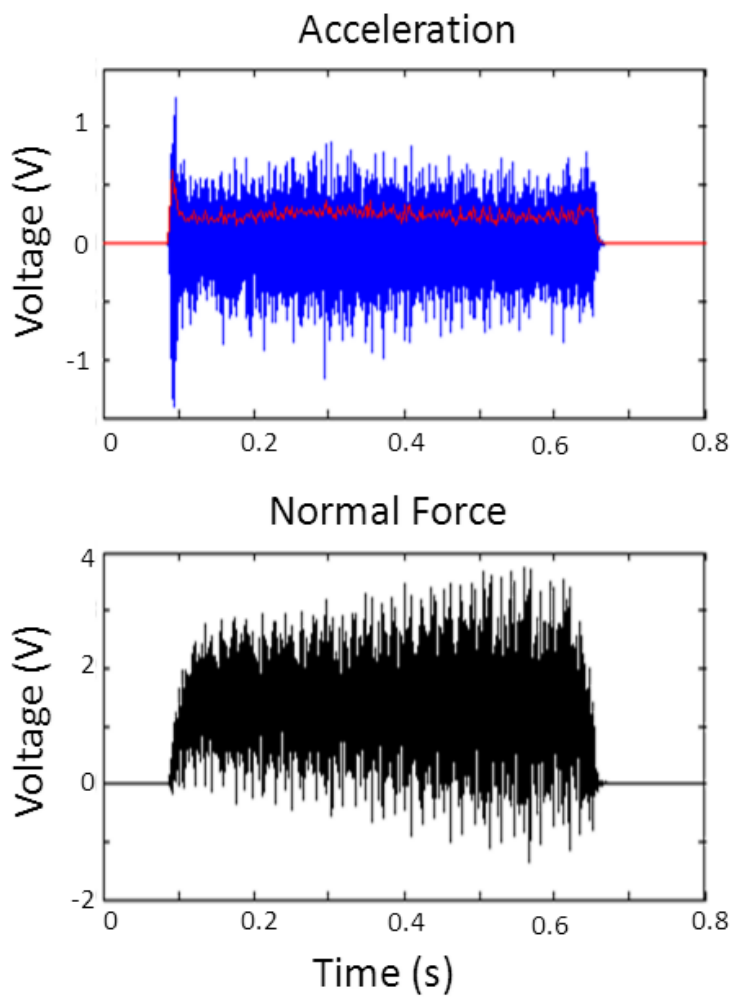

Figure 5.1: Acceleration and normal force raw data. RMS is shown of acceleration data only. Accelerometer sensitivity: $1 \mathrm{~V}=10 \mathrm{~g}$, Dyno sensitivity: $1 \mathrm{~V}=200 \mathrm{~N}$

When comparing the results of each of the two different sensors, entry and exit spike behavior is not as explicit as when compared to AE results. Although the acceleration data does initially spike in amplitude during the engagement of the grinding wheel with the workpiece, it does not exhibit a similar exit repsonse. This may be attributed to the sudden impact or jerk at the entry region, inducing an increased vibration at this area. It must be kept in mind that even though the accelerometer and AE sensors work in a similar manner, their bandwidths are completely different. It is for this reason that vibrations recorded by the accelerometer are generally not recognized by the AE sensor. However, this is not to say that these low frequency vibrations cannot induce a higher frequency acoustic emission during surface grinding [7]. As this work is focused on AE entry and exit spike behavior, more work may be needed to determine if they are at all related to the acceleration response. 
The normal force data displayed in Figure 5.1 does not show any signs of entry/exit spike existenece. Due to its much lower frequency response, it has been found to be far more sensitive to chatter vibration than $\mathrm{AE}[\mathbf{4 0}]$. With chatter frequencies in grinding generally found to lie well under $1000 \mathrm{~Hz}$ [43,39], it is fair to assume that the AE technique is not best suited for detecting this type of phenomenon. It was found that both acceleration and force data were not as directly useful as the corresponding AE results, especially when focusing on entry and exit spike occurrence.

\subsubsection{Workpiece Material}

All of the discussed AE results thus far have corresponded to experiments using HSS workpiece samples. This material was primarily used because AE spikes have been reported to exist during grinding similarly hard steels, and it is comparable to the typical materials ground in industry. However, several experiments were performed on softer steel workpieces as well as complete polymer composites to observe the effect on the AE signal. The results were not conclusive to be useful in terms of AE spike analysis, as the signal became unlike all previous observations. AE spikes were observed in some instances, however, they were not as uniform and repeatable with much softer workpiece materials. When the material properties are changed, the ways in which the acoustic emissions are generated and propagate through the workpiece vary substantially.

It was found that thermal effects as well as the possible damping of the grinding wheel attribute to the AE levels during surface grinding. When completely different grades of steel are used, it becomes difficult to compare the AE features between experiments as each workpiece has individual material properties. Therefore, a future investigation of the effect of various 
workpiece materials would be an area of potential interest. A proposed method of analysis may involve using a single steel workpiece with varying hardness during grinding. The Jominy end quench technique could be employed to produce such a workpiece with variable hardness along its length [44]. By utilizing this method to create a sample workpiece, perhaps the AE data could be characterized as a function of workpiece hardness.

\subsubsection{Dynamic Effects}

Attempts were made to control the level of damping as well as the harmonic frequencies of the system without physically changing the workpiece material or edge geometry. If these factors could be isolated and controlled, then their possible effects on the raw AE data could be determined.

Test concepts involved adjusting the clamping pressure on the workpiece as well as the length of the clamping section in relation to the complete workpiece length. However, the results did not show recognizable changes, possibly suggesting that a new setup be designed to better control these parameters. The biggest issue came with the jaw-length of the grinding vice. In order to compare relative changes in clamping location, a completely new technique must be devised as to keep the AE sensor position uniform between experiments.

This same problem was encountered when performing additional experiments using a cantilever approach; whereby one end of the workpiece was extended to freely overhang from the mounting vice with no vertical support. The setup issue is demonstrated in Figure 5.2. 


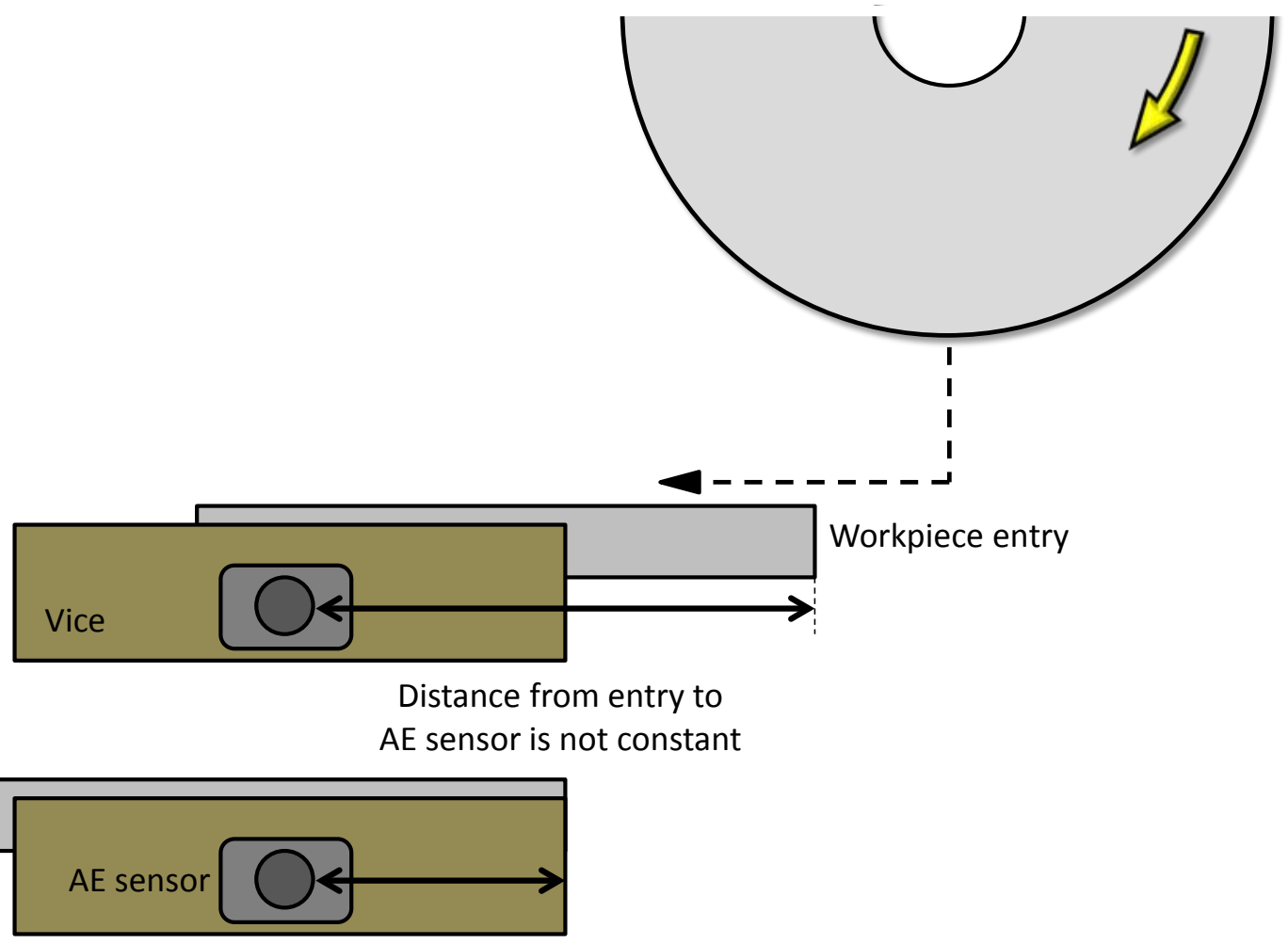

Figure 5.2: Illustration of the setup problem involving static AE sensor positions.

The cantilever lengths were varied and the AE results were observed, however, significant discrepancies were identified when comparing the data. The complete AE signal became non-uniform, dropping off in 'volume' as the cantilever length was increased. As the purpose of this experiment was defined to focus on the possible differences between the raw $\mathrm{AE}$ data within the entry spike regions, comparisons became difficult as the sensor location heavily influenced the result. It must also be noted that simple changes to the sensor location on the vice failed to compensate for this effect between tests, and actually further complicated the comparison process. This is because the path in which the acoustic waves travel from the grinding zone, through the workpiece, to the vice, and finally into the sensor is completely different in this case. Since acoustic emissions follow every rule concerning wave propagation, they can be significantly transformed along the path to the sensor [13]. 
Future work in this area is recommended in order to investigate the effects of dynamic changes to the grinding system on the AE signal. Suggestions on improving the setup include the possible use of a new type of AE senor mounted within the machine spindle itself. Spindle mounted AE sensors are more complicated to implement, but have the advantage of always maintaining a constant distance from the grinding zone.

In conclusion, this work has focused on determining the cause and interpretation of $\mathrm{AE}$ entry and exit spikes during surface grinding. Results have shown a strong relationship between thermal effects and workpiece geometry and how they influence AE spike behavior. Dynamic effects are also believed to contribute to this phenomenon, with a possible association to the level of damping within the system. There are many exciting opportunities for continued research in this area, with results being of great practical significance. Analysis of the raw AE signal has proven to be successful in developing an actual understanding of the entry/exit spike occurrence, but more importantly has helped in gaining further insight into the grinding process. 


\section{References}

[1] Europa Lehrmittel, Fachkunde Metall, 2010.

[2] J. Webster, I. Marinescu, and R. Bennett, "Acoustic Emission for Process Control and Monitoring of Surface Integrity during Grinding," Annals of the CIRP, vol. 43, pp. 299-304, 1994.

[3] D. Lee, I. Hwang, and C. Valente, "Precision manufacturing process monitoring with acoustic emission," International Journal of Machine Tools \& Manufacture, vol. 46, pp. 176-188, 2006.

[4] D. Axinte and N. Gindy, "Tool condition monitoring in broaching," Wear, vol. 254, pp. 370-382, 2003.

[5] W. Hundt, D. Leuenberger, and F. Rehsteiner, "An Approach to Monitoring of the Grinding Process Using Acoustic Emission (AE) Technique," Annals of the CIRP, vol. 43, pp. 295-298, 1994.

[6] M. Surgeon and M. Wevers, "One sensor linear location of acoustic emission events using plate wave theories," Materials Science and Engineering, vol. A265, pp. 254-261, 1999.

[7] Y. Matsumoto, N. Tjiang, B. Foote, and Y. Naerheim, "Tool wear monitoring using acoustic emission in the existence of chatter," International Journal of Production Research, vol. 28, pp. 1861-1869, 1990.

[8] I. Inasaki, "Application of acoustic emission sensor for monitoring machining processes," Ultrasonics, vol. 36, pp. 273-281, 1998.

[9] Physical Acoustics Corporation, PCI-2 Based AE System User's Manual, Rev. 3, 2007. 
[10] J. Webster, W. Dong, and R. Lindsay, "Raw Acoustic Emission Signal Analysis of Grinding Process," Annals of the CIRP, vol. 45, pp. 335-340, 1996.

[11] A. Hassui, A. Diniz, J. Oliveira, J. Felipe, and J. Gomes, "Experimental evaluation on grinding wheel wear through vibration and acoustic emission," Wear, vol. 217, pp. 7-14, 1998.

[12] P. Aguiar, P. Serni, E. Bianchi, and F. Dotto, "In-process grinding monitoring by acoustic emission," Journal of the Brazilian Society of Mechanical Sciences and Engineering, vol. 28, no. 1, pp. 118-124, 2006.

[13] K. Jemielniak, "Some aspects of acoustic emission signal pre-processing," Journal of Materials Processing Technology, vol. 109, pp. 242-247, 2001.

[14] K. Jemielniak and O. Otman, "Catastrophic Tool Failure Detection Based on Acoustic Emission Signal Analysis," Annals of the CIRP, vol. 47, pp. 31-34, 1998.

[15] P. Srinivasa and P. Ramakrishna, "Acoustic emission analysis for tool wear monitoring in face milling," International Journal of Production Research, vol. 40, pp. 1081-1093, 2002.

[16] D. Axinte, N. Gindy, K. Fox, and I. Unanue, "Process monitoring to assist the workpiece surface quality in machining," International Journal of Machine Tools \& Manufacture, vol. 44, pp. 1091-1108, 2004.

[17] D. Axinte, "An experimental analysis of damped coupled vibrations in broaching," International Journal of Machine Tools \& Manufacture, vol. 47, pp. 2182-2188, 2007.

[18] J. Aurich, D. Dornfeld, and P. Arrazola, "Burrs - Analysis, Control and Removal," Annals of the CIRP, vol. 58, pp. 519-542, 2009.

[19] S. Kawamura and J. Yamakawa, "Formation and Growing up Process of Grinding Burrs," 
Bulletin of the Japan Society of Precision Engineering, vol. 23, no. 3, pp. 194-199, 1989.

[20] C. Barth, R. Dollmeier, and G. Warnecke, "Burr Formation in Grinding of Hardened Steel with Conventional and Superabrasive Wheels," SME Technical Paper, pp. 1-6, 2001.

[21] J. Aurich, H. Sudermann, and H. Bil, "Characterisation of Burr Formation in Grinding and Prospects for Modelling," Annals of the CIRP, vol. 54, pp. 313-316, 2005.

[22] S. Van Bohemen and J. Sietsma, "Analysis of acoustic emission signals originating from bainite and martensite formation," Philosophical Magazine, vol. 85, pp. 1791-1804, 2005.

[23] Q. Liu, X. Chen, and N. Gindy, "Investigation of acoustic emission signals under a simulative environment of grinding burn," International Journal of Machine Tools \& Manufacture, vol. 46, pp. 284-292, 2006.

[24] S. Malkin and C. Guo, "Thermal Analysis of Grinding," Annals of the CIRP, vol. 56, pp. 760-782, 2007.

[25] B. Shen, A. Shih, and G. Xiau, "A Heat Transfer Model Based on Finite Difference Method for Grinding," Journal of Manufacturing Science and Engineering, vol. 133, pp. 1-10, 2011.

[26] H. Eda et al., "In-process detection of grinding burn by means of utilizing acoustic emission," Bulletin of the Japan Society of Precision Engineering, vol. 18, pp. 299-304, 1984.

[27] W. Konig and T. Klumpen, "Monitoring and Sensor Concepts for Higher Process Reliablilty," SME Technical Paper, MR93-358, pp. 1-23, 1993.

[28] J. Griffin and X. Chen, "Multiple classification of the acoustic emission signals extracted during burn and chatter anomalies using genetic programming," International Journal of Advanced Manufacturing Technology, vol. 45, pp. 1152-1168, 2009. 
[29] C. Guo and S. Malkin, "Analysis of Transient Temperatures in Grinding," Journal of Engineering for Industry, vol. 117, pp. 571-577, 1995.

[30] R. Snoeys, K. Leuven, and M. Maris, "Thermally induced damage in grinding," Annals of the CIRP, vol. 27, pp. 571-581, 1978.

[31] C. Mao, Z. Zhou, and Y. Ren, "Analysis and FEM Simulation of Temperature Field in Wet Surface Grinding," Materials and Manufacturing Processes, vol. 25, pp. 399-406, 2010.

[32] C. Mao, Z. Zhou, D. Zhou, and D. Gu, "Analysis of Influence Factors for the Contact Length between Wheel and Workpiece in Surface Grinding," Key Engineering Materials, pp. 128-132, 2008.

[33] B. Rowe, Principles of Modern Grinding Technology, 1st ed. Burlington, USA: William Andrew, 2009.

[34] W. Rowe, M. Morgan, H. Qi, and H. Zheng, "The Effect of Deformation on the Contact Area in Grinding," Annals of the CIRP, vol. 42, pp. 409-412, 1993.

[35] H. Qi, W. Rowe, and B. Mills, "Experimental investigation of contact behavior in grinding," Tribology International, vol. 30, pp. 283-294, 1997.

[36] J. Wager and D. Gu, "Influence of Up-Grinding and Down-Grinding on the Contact Zone," Annals of the CIRP, vol. 40, pp. 323-326, 1991.

[37] E. Salje and R. Paulmann, "Relations between abrasive processes," Annals of the CIRP, vol. 32, p. 641, 1988.

[38] T. Yamada, H. Lee, and K. Miura, "Contact Stiffness of Grinding Wheels due to the Difference of Table Feed Rate," Advanced Materials Reaserch, vol. 76-78, pp. 137-142, 2009. 
[39] I. Inasaki, B. Karpushewski, and H. Lee, "Grinding Chatter - Origin and Suppression," Annals of the CIRP, vol. 50, pp. 515-534, 2001.

[40] J. Gradisek, A. Baus, E. Govekar, and F. Kocke, "Automatic chatter detection in grinding," International Journal of Machine Tools \& Manufacture, vol. 43, pp. 1397-1403, 2003.

[41] Kistler Instrument Corporation, Piezotron Sensor: Acoustic Emission Sensor, 2007.

[42] J. Oliveira and C. Valente, "Fast Grinding Process Control with AE Modulated Power Signals," Annals of the CIRP, vol. 53, pp. 267-270, 2004.

[43] E. Govekar, A. Baus, J. Gradisek, F. Klocke, and I. Grabec, "A New Method for Chatter Detection in Grinding," Annals of the CIRP, vol. 51, pp. 267-270, 2002.

[44] P. Koshy and P. Dumitrescu, "A simple technique to characterize the role of work hardness in hard part machining," Journal of Engineering Manufacture, vol. 217, pp. 1485-1489, 2003. 